\title{
Matching Procedure for the Sixth Painlevé Equation (May 2006)
}

\author{
Davide Guzzetti
}

\begin{abstract}
In the framework of the isomonodromy deformation method, we present a constructive procedure to obtain the critical behavior of Painlevé VI transcendents and solve the connection problem. This procedure yields two and one parameter families of solutions, including trigonometric and logarithmic behaviors, and three classes of solutions with Taylor expansion at a critical point.
\end{abstract}

\section{Introduction}

This paper appeared in May 2006. I put it on the archive now, with more that four years of delay, for completeness sake. The paper is published in J.Phys.A: Math.Gen. 39 (2006), 11973-12031, with some modifications. The sixth Painlevé equation is:

$$
\begin{aligned}
& \frac{d^{2} y}{d x^{2}}=\frac{1}{2}\left[\frac{1}{y}+\frac{1}{y-1}+\frac{1}{y-x}\right]\left(\frac{d y}{d x}\right)^{2}-\left[\frac{1}{x}+\frac{1}{x-1}+\frac{1}{y-x}\right] \frac{d y}{d x} \\
& +\frac{y(y-1)(y-x)}{x^{2}(x-1)^{2}}\left[\alpha+\beta \frac{x}{y^{2}}+\gamma \frac{x-1}{(y-1)^{2}}+\delta \frac{x(x-1)}{(y-x)^{2}}\right], \quad \text { (PVI). }
\end{aligned}
$$

The generic solution has essential singularities and/or branch points in $0,1, \infty$. It's behavior at these points will be called critical. The other singularities, which depend on the initial conditions, are poles. A solution of PVI can be analytically continued to a meromorphic function on the universal covering of $\mathbf{P}^{1} \backslash\{0,1, \infty\}$. For generic values of the integration constants and of the parameters $\alpha, \beta, \gamma, \delta$, it cannot be expressed via elementary or classical transcendental functions. For this reason, it is called a Painlevé transcendent. Solving (PVI) means: i) Determine the critical behavior of the transcendents at the critical points $x=0,1, \infty$. Such a behavior must depend on two integration constants. ii) Solve the connection problem, namely: find the relation between couples of integration constants at $x=0,1, \infty$.

We use a matching procedure to study the above two problems. The procedure allows us to compute the first leading terms of the critical behavior at a critical point and the associated monodromy data. This procedure is essentially the isomonodromy deformation method. The reason for our terminology is that we make particular use of the matching between local solutions of two different reductions of the linear system of ODE, associated to (PVI) by the isomonodromy deformation theory. This matching allows us to obtain the leading term(s) of the asymptotic behavior of a corresponding Painlevé transcendent $y(x)$. In this sense, we say that our approach is constructive. Namely, we don't assume any behavior of $y(x)$; rather, we obtain it from the matching condition. This differs from other authors' approach, who start by assuming a given asymptotics for $y(x)$ and then compute the corresponding monodromy data (and so they solve the connection problem). This kind of approach was successfully used for some of the Painlevé equations and allowed many progresses. Our approach is developed to tackle with the cases when we don't know - or we are not able to guess - the asymptotic behavior. In the case of (PVI), we may say that most of the solutions are known. But for some points in the space of monodromy data, we still don't know the corresponding critical behaviors. Our work is motivated by the need to explore these remaining cases.

Once the local matching is done, we proceed with a global description of the solutions of the associate linear system of ODE, in order to compute its monodromy data. These are the monodromy data associated to the solution $y(x)$, of which the asymptotic behavior has been obtained by the precedent step. Again, this computation is done by a (global) matching, among solutions of the two reduced systems and that of the original one. This is the main powerful point of the isomonodromy 
deformation method. The monodromy data are computed in terms of the coefficients of the linear system of ODE, which are elementary functions of the parameters (namely, the integration constants) appearing in the leading term of the asymptotic behavior of $y(x)$. The inversion of the formulae expressing the monodromy data, gives the leading term of $y(x)$ in term of the monodromy data.

The procedure can be repeated at the other singularities $x=1, \infty$. In case of (PVI), $x=0,1, \infty$ are equivalent by symmetry transformations. These facts allow to solve the connection problem $([17],[8],[9],[11],[5]){ }^{1}$

The work of Jimbo [17] is the first on the subject. For generic values of $\alpha, \beta, \gamma \delta$, PVI admits a 2-parameter class of solutions, with the following critical behaviors:.

$$
\begin{gathered}
y(x)=a x^{1-\sigma}\left(1+O\left(|x|^{\epsilon}\right)\right), \quad x \rightarrow 0, \\
y(x)=1-a^{(1)}(1-x)^{1-\sigma^{(1)}}\left(1+O\left(|1-x|^{\epsilon}\right)\right), \quad x \rightarrow 1, \\
y(x)=a^{(\infty)} x^{\sigma^{(\infty)}}\left(1+O\left(|x|^{-\epsilon}\right)\right), \quad x \rightarrow \infty,
\end{gathered}
$$

where $\epsilon$ is a small positive number, $a^{(i)}$ and $\sigma^{(i)}$ are complex numbers such that $a^{(i)} \neq 0$ and $0<\Re \sigma<1,0<\Re \sigma^{(1)}<1,0<\Re \sigma^{(\infty)}<1$. We remark that $x$ converges to the critical points inside a sector with vertex on the corresponding critical point. The connection problem is to finding the relation among the three pairs $(\sigma, a),\left(\sigma^{(1)}, a^{(1)}\right),\left(\sigma^{(\infty)}, a^{(\infty)}\right)$. In [17] the problem is solved by the isomonodromy deformation method. In particular, the exponents are determined by the relations:

$$
2 \cos (\pi \sigma)=\operatorname{tr}\left(M_{0} M_{x}\right), \quad 2 \cos \left(\pi \sigma^{(1)}\right)=\operatorname{tr}\left(M_{1} M_{x}\right), \quad 2 \cos \left(\pi \sigma^{(\infty)}\right)=\operatorname{tr}\left(M_{0} M_{1}\right) .
$$

Here $M_{0}, M_{x}, M_{1}$ are monodromy matrices to be introduced below.

The above class of solutions was enlarged in [26] and [11], to the values $\sigma \in \mathbf{C}, \sigma \notin(-\infty, 0] \cup$ $[1,+\infty$ ) (here we consider $x \rightarrow 0$ ). When $\Re \sigma \geq 1$ or $\Re \sigma \leq 0$, the critical behavior is like the above, but it holds for $x \rightarrow 0$ in a spiral-shaped domain in the universal covering of a punctured neighborhood of $x=0$, along a paths joining a point $x_{0}$ to $x=0$. Along special paths which approach the movable poles, these solution may have behavior $y(x) \sim \sin ^{-2}\left(\frac{i \sigma}{2} \ln x+\varphi(x, a)\right)$, where $\varphi(x, a)$ is a phase depending on the parameter $a$. The transformation $\sigma \mapsto \pm \sigma+2 N, N \in \mathbf{Z}$, leaves the identity $\operatorname{tr}\left(M_{0} M_{x}\right)=2 \cos (\pi \sigma)$ invariant. Its effect on the solutions is studied in [11]. As a result, one can reduce to the values $0 \leq \Re \sigma \leq 1, \sigma \neq 0,1$. The reader may find a synthetic description of these results in the review paper [12].

It is an open problem to determine the critical behavior, say at $x=0$, for $\sigma=0,1$. To be more precise, the problem is encountered when $\operatorname{tr}\left(M_{i} M_{j}\right)= \pm 2$. These are precisely the points of the space of monodromy data mentioned above, in correspondence of which we do not know the critical behavior. In addition, certain non-generic values of $\alpha, \beta, \gamma, \delta$ are not yet studied. The matching procedure is motivated by the need to explore these unknown cases.

As a result of the matching procedure, we obtain:

R1) A two-parameter family of solutions, of the type found by Jimbo [17]. Besides, we show that there are solutions with trigonometric behavior.

R2) One-parameter families of solutions, including a class of logarithmic solutions.

Together with the results of [26] and [11], R1) and R2) will cover all cases $\operatorname{tr}\left(M_{i} M_{j}\right) \neq-2$, namely $\sigma \neq 1$. By symmetry transformations, some of the cases $\operatorname{tr}\left(M_{i} M_{j}\right)=-2$ can be obtained from the above results (for example, the Chazy solutions [21]).

R3) The solutions which admit a Taylor expansion at $x=0$.

R4) We compute the corresponding monodromy data.

\footnotetext{
${ }^{1}$ For reasons of space, we limit ourselves to the computation of monodromy data, without explaining how the connection problem is practically solved once the monodromy data are computed, and how the analytic continuation is done. We refer the reader to [17], [8], [9], [11], [5]. The behaviors at $x=1$ and $x=\infty$, and the dependence of them on the monodromy data are deduced from the behavior at $x=0$ by symmetry transformations. PVI is invariant for the change of variables $y(x)=1-\tilde{y}(t), x=1-t$ and simultaneous permutation of $\theta_{0}, \theta_{1}$. This means that $y(x)$ solves PVI if and only if $\tilde{y}(t)$ solves PVI with permuted parameters and independent variable $t$. Similarly, PVI is invariant for $y(x)=1 / \tilde{y}(t), x=1 / t$ and simultaneous permutation of $\theta_{\infty}, \theta_{0}$. It is invariant for $y(x)=(\tilde{y}(t)-t) /(1-t)$, $x=t /(t-1)$ and simultaneous permutation of $\theta_{0}, \theta_{x}$. By composing the third, first and again third symmetries, we get $y(x)=\tilde{y}(t) / t, t=1 / x$ with the permutation of $\theta_{1}, \theta_{x}$. Therefore, the critical points $0,1, \infty$ are equivalent.
} 
In virtue of the symmetries of (PVI) (birational transformations of $(x, y(x))$ ), it can be shown that the solutions with Taylor expansion at $x=0$, obtained by the matching procedure, are the representatives of three equivalent classes, which include all the solutions admitting a Taylor expansion at a critical point. If we define $\sigma$ through the relation $\operatorname{tr}\left(M_{0} M_{x}\right)=2 \cos (\pi \sigma)$, the representatives of three equivalent classes correspond to values $\sigma=0, \sigma= \pm\left(\theta_{1} \pm / \mp \theta_{\infty}\right)$ and $\sigma=1$.

A further step in the study of PVI, is the problem of the systematic classification of all the solutions of (PVI) in terms of the monodromy data of the associated linear system. As we discussed above, the matching procedure is effective to produce new solutions, associated to monodromy data for which the connection problem has not yet been studied. Therefore, it is a tool to study the classification problem. This classification will be done in another paper.

A matching procedure, to obtain asymptotic behaviors and monodromy data in the framework of the isomonodromy deformation method, was suggested by Its and Novokshenov in [15], for the second and third Painlevé equations. The work by Jimbo [17] can be regarded as an implicit matching procedure. This method was further developed and used by Kapaev, Kitaev, Andreev, and Vardanyan. Here we cite the case of the fifth Painlevé equation, in [2]. An analogous matching scheme is used in [1], for a different problem (limit PVI $\rightarrow$ PV).

Acknowledgements (May 2006): The author wishes to thank Alexander Kitaev for introducing him to the matching procedure and for many discussions. This paper was written in RIMS, Kyoto University, supported by the Kyoto Mathematics COE fellowship.

\section{PART I: Matching Procedure and Results}

\section{Matching Procedure}

PVI is the isomonodromy deformation equation of a Fuchsian system of differential equations [18]:

$$
\frac{d \Psi}{d \lambda}=A(\lambda, x, \theta) \Psi, \quad A(\lambda, x, \theta):=\left[\frac{A_{0}(x, \theta)}{\lambda}+\frac{A_{x}(x, \theta)}{\lambda-x}+\frac{A_{1}(x, \theta)}{\lambda-1}\right], \quad \lambda \in \mathbf{C} .
$$

The $2 \times 2$ matrices $A_{i}(x, \theta)$ depend on $x$ in such a way that the monodromy of a fundamental solution $\Psi(\lambda, x)$ does not change for small deformations of $x$. They also depend on the parameters $\alpha, \beta, \gamma, \delta$ of PVI through more elementary parameters $\theta=\left(\theta_{0}, \theta_{x}, \theta_{1}, \theta_{\infty}\right)$ according to the following relations:

$$
\begin{gathered}
-A_{\infty}:=A_{0}+A_{1}+A_{x}=-\frac{\theta_{\infty}}{2} \sigma_{3}, \quad \theta_{\infty} \neq 0 . \quad \text { Eigenvalues }\left(A_{i}\right)= \pm \frac{1}{2} \theta_{i}, \quad i=0,1, x ; \\
\alpha=\frac{1}{2}\left(\theta_{\infty}-1\right)^{2}, \quad-\beta=\frac{1}{2} \theta_{0}^{2}, \quad \gamma=\frac{1}{2} \theta_{1}^{2}, \quad\left(\frac{1}{2}-\delta\right)=\frac{1}{2} \theta_{x}^{2}
\end{gathered}
$$

Here $\sigma_{3}$ is the Pauli matrix. The equations of monodromy-preserving deformation (Schlesinger equations), can be written in Hamiltonian form and reduce to PVI, being the transcendent $y(x)$ solution of $A(y(x), x, \theta)_{1,2}=0$. Namely:

$$
y(x)=\frac{x\left(A_{0}\right)_{12}}{x\left[\left(A_{0}\right)_{12}+\left(A_{1}\right)_{12}\right]-\left(A_{1}\right)_{12}},
$$

The matrices $A_{i}(x, \theta), i=0, x, 1$, depend on $y(x), \frac{d y(x)}{d x}$ and $\int y(x)$ through rational functions, which are given in [18]. In short, we will write $A_{i}=A_{i}(x)$.

The product of the monodromy matrices $M_{0}, M_{x}, M_{1}$ of a fundamental matrix solution $\Psi$ at $\lambda=0, x, 1$ respectively, is equal to the monodromy at $\lambda=\infty$. The order of the producs depends on the choice of a basis of loops. As a consequence, the following relation must hold:

$$
\begin{gathered}
\cos \left(\pi \theta_{0}\right) \operatorname{tr}\left(M_{1} M_{x}\right)+\cos \left(\pi \theta_{1}\right) \operatorname{tr}\left(M_{0} M_{x}\right)+\cos \left(\pi \theta_{x}\right) \operatorname{tr}\left(M_{1} M_{0}\right) \\
=2 \cos \left(\pi \theta_{\infty}\right)+4 \cos \left(\pi \theta_{1}\right) \cos \left(\pi \theta_{0}\right) \cos \left(\pi \theta_{x}\right) .
\end{gathered}
$$




\subsection{Leading Terms of $y(x)$ as a result of Matching}

We present the constructive procedure to obtain the leading terms of a solution $y(x)$, when $x \rightarrow 0$. This procedure has been used for the fifth Painlevé equation by F.V. Andreev and A.V. Kitaev in [2]. An analogous scheme is used in [1], for a different problem. In particular, in [1] the non fuchsian singularity in the $\Psi_{O U T}$-equation (to be introduced below) appears.

Since we are considering $x \rightarrow 0$, we divide the $\lambda$-plane into two domains. The "outside" domain is defined for $\lambda$ sufficiently big:

$$
|\lambda| \geq|x|^{\delta_{O U T}}, \quad \delta_{\text {OUT }}>0 .
$$

Therefore, (4) can be written as:

$$
\frac{d \Psi}{d \lambda}=\left[\frac{A_{0}+A_{x}}{\lambda}+\frac{A_{x}}{\lambda} \sum_{n=1}^{\infty}\left(\frac{x}{\lambda}\right)^{n}+\frac{A_{1}}{\lambda-1}\right] \Psi .
$$

The "inside" domain is defined for $\lambda$ comparable with $x$, namely:

$$
|\lambda| \leq|x|^{\delta_{I N}}, \quad \delta_{I N}>0 .
$$

Therefore, $\lambda \rightarrow 0$ as $x \rightarrow 0$, and we rewrite (4) as:

$$
\frac{d \Psi}{d \lambda}=\left[\frac{A_{0}}{\lambda}+\frac{A_{x}}{\lambda-x}-A_{1} \sum_{n=0}^{\infty} \lambda^{n}\right] \Psi .
$$

If the behavior of $A_{0}(x), A_{1}(x)$ and $A_{x}(x)$ is sufficiently good, we expect that the higher order terms in the series of (8) and (10) are small corrections which can be neglected when $x \rightarrow 0$. If this is the case, (8) and (10) reduce respectively to:

$$
\begin{gathered}
\frac{d \Psi_{\text {OUT }}}{d \lambda}=\left[\frac{A_{0}+A_{x}}{\lambda}+\frac{A_{x}}{\lambda} \sum_{n=1}^{N_{\text {OUT }}}\left(\frac{x}{\lambda}\right)^{n}+\frac{A_{1}}{\lambda-1}\right] \Psi_{\text {OUT }}, \\
\frac{d \Psi_{I N}}{d \lambda}=\left[\frac{A_{0}}{\lambda}+\frac{A_{x}}{\lambda-x}-A_{1} \sum_{n=0}^{N_{I N}} \lambda^{n}\right] \Psi_{I N},
\end{gathered}
$$

where $N_{I N}, N_{O U T}$ are suitable integers. The simplest reduction is to Fuchsian systems:

$$
\begin{gathered}
\frac{d \Psi_{O U T}}{d \lambda}=\left[\frac{A_{0}+A_{x}}{\lambda}+\frac{A_{1}}{\lambda-1}\right] \Psi_{O U T}, \\
\frac{d \Psi_{I N}}{d \lambda}=\left[\frac{A_{0}}{\lambda}+\frac{A_{x}}{\lambda-x}\right] \Psi_{I N} .
\end{gathered}
$$

It is a new feature of this paper that we will use reduced non-fuchsian systems. In the literature, the fuchsian reduction has been privileged, but we show that in some relevant cases it cannot be used, being the non-fuchsian reduction necessary.

Generally speaking, we can parameterize the elements of $A_{0}+A_{x}$ and $A_{1}$ of (13) in terms of $\theta_{1}$, the eigenvalues of $A_{0}+A_{x}$ and the eigenvalues $\theta_{\infty}$ of $A_{0}+A_{x}+A_{1}$. We also need an additional unknown function of $x$. In the same way, we can explicitly parameterize the elements of $A_{0}$ and $A_{x}$ in (14) in terms of $\theta_{0}, \theta_{x}$, the eigenvalues of $A_{0}+A_{x}$ and another additional unknown function of $x$. When the reductions (11) and (12) are non-fuchsian, particular care must be payed. This will be explained case by case in the paper. Our purpose is to find the leading term of the unknown functions when $x \rightarrow 0$, in order to determine the critical behavior of $A_{0}(x), A_{1}(x), A_{x}(x)$ and (6).

The leading term can be obtained as a result of two facts:

i) Systems (11) and (12) are isomonodromic. This imposes constraints on the form of the unknown functions. Typically, one of them must be constant.

ii) Two fundamental matrix solutions $\Psi_{O U T}(\lambda, x), \Psi_{I N}(\lambda, x)$ must match in the region of overlap, provided this is not empty:

$$
\Psi_{O U T}(\lambda, x) \sim \Psi_{I N}(\lambda, x), \quad|x|^{\delta_{O U T}} \leq|\lambda| \leq|x|^{\delta_{I N}}, \quad x \rightarrow 0
$$


This relation is to be intended in the sense that the leading terms of the local behavior of $\Psi_{\text {OUT }}$ and $\Psi_{I N}$ for $x \rightarrow 0$ must be equal. This determines a simple relation between the two functions of $x$ appearing in $A_{0}, A_{x}, A_{1}, A_{0}+A_{x}$. (15) also implies that $\delta_{I N} \leq \delta_{O U T}$.

Practically, to fulfill point ii), we will match a fundamental solution of (11) for $\lambda \rightarrow 0$, with a fundamental solution of (12) when $\mu:=\lambda / x \rightarrow \infty$, namely with a solution of:

$$
\frac{d \Psi_{I N}}{d \mu}=\left[\frac{A_{0}}{\mu}+\frac{A_{x}}{\mu-1}-x A_{1} \sum_{n=0}^{N_{I N}} x^{n} \mu^{n}\right] \Psi_{I N}, \quad \mu:=\frac{\lambda}{x} .
$$

To summarize, matching two fundamental solutions of the reduced isomonodromic systems (11) and (12), we obtain the leading term(s), for $x \rightarrow 0$, of the entries of the matrices of the original system (4). The procedure is algorithmic, no a priori assumption about the behavior being necessary.

This method is sometimes called coalescence of singularities, because the singularity $\lambda=0$ and $\lambda=x$ coalesce to produce system (11), while the singularity $\mu=\frac{1}{x}$ and $\mu=\infty$ coalesce to produce system (16). Coalescence of singularities was first used by M. Jimbo in [17] to compute the monodromy matrices of (4) for a class of solutions of (PVI) with leading term $y(x) \sim a x^{1-\sigma}$, $0<\Re \sigma<1$.

\subsection{Computation of the Monodromy Data}

Let $\Psi$ be a fundamental matrix solution of (4), and let $M_{0}, M_{x}, M_{1}, M_{\infty}$ be its monodromy matrices at $\lambda=0, x, 1, \infty$ respectively $\left(M_{\infty}\right.$ is the product of $M_{0}, M_{x}, M_{1}$, the order depending on the choice of a basis of loops). As a consequence of isomonodromicity, there exists a fundamental solution $\Psi_{O U T}$ of (11) such that

$$
M_{1}^{\text {OUT }}=M_{1}, \quad M_{\infty}^{\text {OUT }}=M_{\infty},
$$

where $M_{1}^{\text {OUT }}$ and $M_{\infty}^{\text {OUT }}$ are the monodromy matrices of $\Psi_{\text {OUT }}$ at $\lambda=1, \infty$. Moreover, $M_{0}^{\text {OUT }}=$ $M_{0} M_{x}$ or $M_{x} M_{0}$, depending on the order of loops. A detailed proof of these facts can be found in [9]. There also exists a fundamental solution $\Psi_{I N}$ of (12) such that:

$$
M_{0}^{I N}=M_{0}, \quad M_{x}^{I N}=M_{x},
$$

where $M_{0}^{I N}$ and $M_{x}^{I N}$ are the monodromy matrices of $\Psi_{I N}$ at $\lambda=0, x$.

The method of coalescence of singularities is useful when the monodromy of the reduced systems (11), (12) can be explicitly computed. This is the case when the reduction is fuchsian (namely (13), (14)), because fuchsian systems with three singular points are equivalent to a Gauss hypergeometric equation (see Appendix 1). For the non-fuchsian reduction, in general we can compute the monodromy when (11), (12) are solvable in terms of special or elementary functions. This will be discussed case by case in the paper.

In order for this procedure to work, not only $\Psi_{O U T}$ and $\Psi_{I N}$ must match with each other, as in subsection 2.1, but also $\Psi_{O U T}$ must match with a fundamental matrix solution $\Psi$ of (4) in a domain of the $\lambda$ plane, and $\Psi_{I N}$ must match with the same $\Psi$ in another domain of the $\lambda$ plane.

The standard choice of $\Psi$ is as follows:

$$
\Psi(\lambda)=\left\{\begin{array}{cc}
{\left[I+O\left(\frac{1}{\lambda}\right)\right] \lambda^{-\frac{\theta_{\infty}}{2} \sigma_{3}} \lambda^{R_{\infty}},} & \lambda \rightarrow \infty ; \\
\psi_{0}(x)[I+O(\lambda)] \lambda^{\frac{\theta_{0}}{2} \sigma_{3}} \lambda^{R_{0}} C_{0}, & \lambda \rightarrow 0 ; \\
\psi_{x}(x)[I+O(\lambda-x)](\lambda-x)^{\frac{\theta_{x}}{2} \sigma_{3}}(\lambda-x)^{R_{x}} C_{x}, & \lambda \rightarrow x ; \\
\psi_{1}(x)[I+O(\lambda-1)](\lambda-1)^{\frac{\theta_{1}}{2} \sigma_{3}}(\lambda-1)^{R_{1}} C_{1}, & \lambda \rightarrow 1 ;
\end{array}\right.
$$

Here $\psi_{0}(x), \psi_{x}(x), \psi_{1}(x)$ are the diagonalizing matrices of $A_{0}(x), A_{1}(x), A_{x}(x)$ respectively. They are defined by multiplication to the right by arbitrary diagonal matrices, possibly depending on $x$. 
$C_{\kappa}, \kappa=\infty, 0, x, 1$, are invertible connection matrices, independent of $x$ [18]. Each $R_{\kappa}, \kappa=\infty, 0, x, 1$, is also independent of $x$, and:

$$
R_{\kappa}=0 \text { if } \theta_{\kappa} \notin \mathbf{Z}, \quad R_{\kappa}= \begin{cases}\left(\begin{array}{ll}
0 & * \\
0 & 0
\end{array}\right), & \text { if } \theta_{\kappa}>0 \text { integer } \\
\left(\begin{array}{ll}
0 & 0 \\
* & 0
\end{array}\right), & \text { if } \theta_{\kappa}<0 \text { integer }\end{cases}
$$

If $\theta_{i}=0, i=0, x, 1$, then $R_{i}$ is to be considered the Jordan form $\left(\begin{array}{ll}0 & 1 \\ 0 & 0\end{array}\right)$ of $A_{i}$. If $\theta_{\infty}=0, R_{\infty}=0$. Note that for the loop $\lambda \mapsto \lambda e^{2 \pi i},|\lambda|>\max \{1,|x|\}$, we immediately compute the monodromy at infinity:

$$
M_{\infty}=\exp \left\{-i \pi \theta_{\infty}\right\} \exp \left\{2 \pi i R_{\infty}\right\}
$$

Let $\Psi_{O U T}$ and $\Psi_{I N}$ be the solutions of (11) and (12) matching as in (15). We explain how they are matched with (17).

(*) Matching $\Psi \leftrightarrow \Psi_{O U T}$ :

$\lambda=\infty$ is a fuchsian singularity of (11), with residue $-A_{\infty} / \lambda$. Therefore, we can always find a fundamental matrix solution with behavior:

$$
\Psi_{\text {OUT }}^{\text {Match }}=\left[I+O\left(\frac{1}{\lambda}\right)\right] \lambda^{-\frac{\theta_{\infty}}{2} \sigma_{3}} \lambda^{R_{\infty}}, \quad \lambda \rightarrow \infty .
$$

This solution matches with $\Psi$. Also $\lambda=1$ is a fuchsian singularity of (11). Therefore, we have:

$$
\Psi_{\text {OUT }}^{\text {Match }}=\psi_{1}^{\text {OUT }}(x)[I+O(\lambda-1)](\lambda-1)^{\frac{\theta_{1}}{2} \sigma_{3}}(\lambda-1)^{R_{1}} C_{1}^{\text {OUT }}, \quad \lambda \rightarrow 1 ;
$$

Here $C_{1}^{\text {OUT }}$ is a suitable connection matrix. $\psi_{1}^{O U T}(x)$ is the matrix that diagonalizes the leading terms of $A_{1}(x)$. Therefore, $\psi_{1}(x) \sim \psi_{1}^{\text {OUT }}(x)$ for $x \rightarrow 0$. As a consequence of isomonodromicity, $R_{1}$ is the same of $\Psi$.

As a consequence of the matching $\Psi \leftrightarrow \Psi_{O U T}^{M a t c h}$, the monodromy of $\Psi$ at $\lambda=1$ is:

$$
M_{1}=C_{1}^{-1} \exp \left\{i \pi \theta_{1} \sigma_{3}\right\} \exp \left\{2 \pi i R_{1}\right\} C_{1}, \quad \text { with } C_{1} \equiv C_{1}^{\text {OUT }} \text {. }
$$

We finally need an invertible connection matrix $C_{O U T}$ to connect $\Psi_{O U T}^{M a t c h}$ with the solution $\Psi_{O U T}$ appearing in (15). Namely, $\Psi_{O U T}^{M a t c h}=\Psi_{O U T} C_{O U T}$.

(*) Matching $\Psi \leftrightarrow \Psi_{I N}$ :

As a consequence of the matching $\Psi \leftrightarrow \Psi_{\text {OUT }}^{M a t c h}$, we have to choose the IN-solution which matches with $\Psi_{O U T}^{M a t c h}$. This is $\Psi_{I N}^{M a t c h}:=\Psi_{I N} C_{O U T}$.

Now, $\lambda=0, x$ are fuchsian singularities of (12). Therefore:

$$
\Psi_{I N}^{M a t c h}=\left\{\begin{array}{cl}
\psi_{0}^{I N}(x)[I+O(\lambda)] \lambda^{\frac{\theta_{0}}{2} \sigma_{3}} \lambda^{R_{0}} C_{0}^{I N}, & \lambda \rightarrow 0 ; \\
\psi_{x}^{I N}(x)[I+O(\lambda-x)](\lambda-x)^{\frac{\theta_{x}}{2} \sigma_{3}}(\lambda-x)^{R_{x}} C_{x}^{I N}, & \lambda \rightarrow x ;
\end{array}\right.
$$

The above hold for fixed small $x \neq 0$. Here $C_{0}^{I N}$ and $C_{x}^{I N}$ are suitable connection matrices. $\psi_{0}^{I N}(x)$ and $\psi_{x}(x)^{I N}$ are diagonalizing matrices of the leading terms of $A_{0}(x)$ and $A_{x}(x)$. For $x \rightarrow 0$ they match with $\psi_{0}(x)$ and $\psi_{x}(x)$ of $\Psi$ in (17). On the other hand, as a consequence of isomonodromicity, the matrices $R_{0}$ and $R_{x}$ are the same of $\Psi$.

By virtue of the matching $\Psi \leftrightarrow \Psi_{I N}^{M a t c h}$, the connection matrices $C_{0}$ and $C_{x}$ coincide with the $x$-independent connection matrices $C_{0}^{I N}, C_{x}^{I N}$ respectively. As a result, we obtain the monodromy matrices for $\Psi$ :

$$
\begin{array}{ll}
M_{0}=C_{0}^{-1} \exp \left\{i \pi \theta_{0} \sigma_{3}\right\} \exp \left\{2 \pi i R_{0}\right\} C_{0}, & C_{0} \equiv C_{0}^{I N}, \\
M_{x}=C_{x}{ }^{-1} \exp \left\{i \pi \theta_{x} \sigma_{3}\right\} \exp \left\{2 \pi i R_{x}\right\} C_{x}, & C_{x} \equiv C_{x}^{I N} .
\end{array}
$$

Our reduction is useful if the connection matrices $C_{1}^{O U T}, C_{0}^{I N}, C_{x}^{I N}$ can be computed explicitly. This is possible for the fuchsian reduced systems (13), (14). For non-fuchsian reduced systems, we will discuss the computability case by case. 


\section{Results}

In the following, it is understood that $x \rightarrow 0$ inside a sector. Namely, $\arg (x)$ is bounded.

\subsection{Critical Behaviors: Result R3).}

The novelty of this paper is that the matching procedure is applied to non-fuchsian systems (11) and (12). As a result, we obtain all the solutions that admit a Taylor expansion

$$
y(x)=b_{0}+b_{1} x+b_{2} x^{2}+\ldots=\sum_{n=0}^{\infty} b_{n} x^{n}, \quad x \rightarrow 0 .
$$

Precisely, we obtain the representative solutions of three equivalence classes, the equivalence relation being the birational transformations [24] of Appendix 3 and formula (22). Our result is the following.

Theorem 1 The solutions of (PVI) with Taylor expansion at $x=0$ are divided into four equivalent classes (one being that of singular solutions $y=0,1, x$ ). The representatives can be chosen as follows:

1) Singular solution $y=1$.

2) $\theta_{\infty} \neq 1, \theta_{1}-\theta_{\infty} \notin \mathbf{Z}$ [representative of $\theta_{1} \pm \theta_{\infty} \notin \mathbf{Z}$ ]:

$$
y(x)=\frac{\theta_{1}-\theta_{\infty}+1}{1-\theta_{\infty}}+\frac{\theta_{1}\left[\left(\theta_{1}-\theta_{\infty}\right)\left(\theta_{1}-\theta_{\infty}+2\right)+\theta_{x}^{2}-\theta_{0}^{2}\right]}{2\left(\theta_{\infty}-1\right)\left(\theta_{\infty}-\theta_{1}\right)\left(\theta_{\infty}-\theta_{1}-2\right)} x+\sum_{n=3}^{\infty} b_{n}\left(\theta_{1}, \theta_{\infty}, \theta_{0}, \theta_{x}\right) x^{n} .
$$

The coefficients are certain rational functions of $\theta_{0}, \theta_{\infty}, \theta_{0}, \theta_{x}$.

3) $\theta_{1}=\theta_{\infty} \neq 1, \theta_{0}= \pm \theta_{x} \quad\left[\right.$ representative of $\left.\theta_{1} \pm \theta_{\infty} \in \mathbf{Z}, \theta_{x} \pm \theta_{0} \in \mathbf{Z}\right]$ :

$$
y(x)=\frac{1}{1-\theta_{\infty}}+a x+\sum_{n=2}^{\infty} b_{n}\left(a ; \theta_{0}, \theta_{\infty}\right) x^{n} .
$$

The coefficients are certain rational functions of $\theta_{0}, \theta_{\infty}$ and a parameter $a \in \mathbf{C}$.

4) $\theta_{\infty}=1, \theta_{1}=0$ [representative of $\left.\theta_{1} \pm \theta_{\infty} \in \mathbf{Z}, \theta_{\infty} \in \mathbf{Z} \backslash\{0\}\right]$ :

$$
y(x)=a+\frac{1-a}{2}\left(1+\theta_{0}^{2}-\theta_{x}^{2}\right) x+\sum_{n=2}^{\infty} b_{n}\left(a ; \theta_{0} ; \theta_{x}\right) x^{n} .
$$

The coefficients are certain rational functions of $\theta_{0}, \theta_{x}$ and a parameter $a \in \mathbf{C}$.

The monodromy data associated to the above solutions is given in theorem 3 .

The symmetry $\theta_{1} \mapsto-\theta_{1}$, which leaves (PVI) invariant, transforms (18) into:

$$
y(x)=\frac{\theta_{1}+\theta_{\infty}-1}{\theta_{\infty}-1}+\frac{\theta_{1}\left[\left(\theta_{1}+\theta_{\infty}\right)\left(\theta_{1}+\theta_{\infty}-2\right)+\theta_{x}^{2}-\theta_{0}^{2}\right]}{2\left(1-\theta_{\infty}\right)\left(\theta_{\infty}+\theta_{1}\right)\left(\theta_{\infty}+\theta_{1}-2\right)} x+\sum_{n=3}^{\infty} b_{n}\left(-\theta_{1}, \theta_{\infty}, \theta_{0}, \theta_{x}\right) x^{n} .
$$

Here $\theta_{\infty} \neq 1, \theta_{1}+\theta_{\infty} \notin \mathbf{Z}$. The coefficients $b_{n}$ are the same of (18).

The convergence of the Taylor series can be proved by a Briot-Bouquet like argument. This will not be done here, for reasons of space. The reader can find the general procedure in [14] and an application to the fifth Painlevé equation in [20]

\section{Comments:}

1) Characterization of solutions $y(x)=\sum_{n=0}^{\infty} b_{n} x^{n}, b_{0} \neq 0$.

(a) There always exists one solution (18) when $\theta_{1}-\theta_{\infty} \notin \mathbf{Z}$; there always exists one solution (21) when $\theta_{1}+\theta_{\infty} \notin \mathbf{Z}$. The coefficients $b_{n}$ depend rationally on $\theta_{\kappa}, \kappa=0, x, 1, \infty$. (b) There is a one-parameter family of solutions equivalent to (19), when $\theta_{1} \pm \theta_{\infty} \in \mathbf{Z}$ and $\theta_{0} \pm \theta_{x}$ has a particular 
integer value. The coefficients $b_{n}$ depend rationally on a complex parameter $a$ and $\theta_{\infty}, \theta_{0}$. (c) Finally, there is a one-parameter family of solutions equivalent to (20), when $\theta_{1} \pm \theta_{\infty} \in \mathbf{Z}$, and $\theta_{\infty}$ has a particular integer value; the coefficients $b_{n}$ depend rationally on a complex parameter $a$ and $\theta_{0}, \theta_{x}$. The singular solutions $y=0,1, x$ are possibly obtained by birational transformations of (18), (19), (20).

The coefficients $b_{n}$ can always be computed recursively by direct substitution into (PVI). We will clarify these facts by some examples in Appendix 4.

2) Characterization of solutions $y(x)=\sum_{n=1}^{\infty} b_{n} x^{n}, b_{1} \neq 0$.

These solutions are obtained from those of theorem 1 by the symmetry.

$$
\theta_{x} \mapsto \theta_{1}, \quad \theta_{0} \mapsto \theta_{\infty}-1, \quad \theta_{1} \mapsto \theta_{x}, \quad \theta_{\infty} \mapsto \theta_{0}+1 ; \quad y(x) \mapsto \frac{x}{y(x)}
$$

The solutions obtained from the singular solution $y=1$ and (18), (19), (20) are respectively:

1) Singular solution $y(x)=x$.

2) $\theta_{0} \neq 0, \theta_{0} \pm \theta_{x} \notin \mathbf{Z}:$

$$
y(x)=\frac{\theta_{0}}{\theta_{0} \pm \theta_{x}} x \pm \frac{\theta_{0} \theta_{x}\left[\left(\theta_{0} \pm \theta_{x}\right)^{2}+\theta_{1}^{2}-\theta_{\infty}^{2}+2 \theta_{\infty}-2\right]}{2\left(\theta_{0} \pm \theta_{x}\right)^{2}\left[\left(\theta_{0} \pm \theta_{x}\right)^{2}-1\right]} x^{2}+\sum_{n=3}^{\infty} b_{n}\left(\theta_{0}, \theta_{x}, \theta_{1}, \theta_{\infty}\right) x^{n} .
$$

3) $\theta_{0}+\theta_{x}=1, \theta_{0} \neq 0, \theta_{1}= \pm\left(\theta_{\infty}-1\right)$ :

$$
y(x)=\theta_{0} x+a x^{2}+\sum_{n=3}^{\infty} b_{n}\left(a ; \theta_{0}, \theta_{\infty}\right) x^{n} .
$$

4) $\theta_{x}=\theta_{0}=0$.

$$
y(x)=a x+\frac{a(a-1)}{2}\left(\theta_{1}^{2}-\left(\theta_{\infty}-1\right)^{2}-1\right) x^{2}+\sum_{n=3}^{\infty} b_{n}\left(a ; \theta_{1}, \theta_{\infty}\right) x^{n} .
$$

(a) (PVI) has always one or both solutions (23) when $\theta_{0} \pm \theta_{x} \notin \mathbf{Z}$. Also when $\theta_{0}+\theta_{x}$ (or $\theta_{0}-\theta_{x}$ ) is integer, (PVI) has a solution (23) corresponding to $\theta_{0}-\theta_{x}$ not integer (or $\theta_{0}+\theta_{x}$ not integer). (b) When $\theta_{0}+\theta_{x}$ or $\theta_{0}-\theta_{x}$ is integer, (PVI) has a 1-parameter family of solutions equivalent (by birational transformations) to (24); this family exists provided that $\theta_{1} \pm \theta_{\infty}$ has a particular integer value. (c) When $\theta_{0}+\theta_{x}$ or $\theta_{0}-\theta_{x}$ is integer and $\theta_{0}$ has a particular integer value, there is a one parameter family of solutions equivalent to (25).

3) (PVI) has a one-parameter family of solutions of the type:

$$
y(x)=y_{0}(x)+y_{1}(x) a x^{\omega}+y_{2}(x)\left(a x^{\omega}\right)^{2}+\ldots=\sum_{N=0}^{\infty} y_{N}(x)\left(a x^{\omega}\right)^{N}, \quad x \rightarrow 0 ;
$$

where the parameter is $a \in \mathbf{C}$, and the $y_{N}(x)$ 's are Taylor series:

$$
y_{N}(x)=\sum_{k=0}^{\infty} b_{k, N}\left(\theta_{1}, \theta_{\infty}, \theta_{0}, \theta_{x}\right) x^{k}, \quad x \rightarrow 0 .
$$

Either $y_{0}(x)$ is (21) and $\omega= \pm\left(\theta_{1}+\theta_{\infty}-1\right)$, or $y_{0}(x)$ is (18) and $\omega= \pm\left(\theta_{\infty}-\theta_{1}-1\right)$. The conditions $|\Re \omega|<1, \omega \neq 0$ hold. The coefficients $b_{k, N}\left(\theta_{1}, \theta_{\infty}, \theta_{0}, \theta_{x}\right)$ are certain rational functions that can be recursively determined by direct substitution into (PVI). These solutions are the immages of solutions (28) and (29) respectively, through the symmetry (22). Solutions (28) and (29) are a sub-case of theorem 2, obtained by the matching procedure.

Taylor solutions (18), (21) are a special case of (26), when the parameter is zero. Solutions (19) and (20) - and their images by symmetry - are one parameters families of type (26), in non generic cases when $\omega \in \mathbf{Z}$. 
Further study of one-parameter solutions, including non-generic cases when $\theta_{\nu}$ and/or some sum of two $\theta_{\nu}$ 's are integer (including logarithmic one parameter families), will be presented in another paper devoted to the general classification problem.

4) Solutions (18) and the equivalent solutions (21), (23) were also derived in [19] by substitution of a Taylor expansion in (PVI). The corresponding monodromy was computed by coalescence of singularities of a Heun's type (scalar) equation.

\subsection{Critical Behaviors: Results R1), R2).}

We now consider cases when (4) can be reduced to the fuchsian systems (13) and (14). Let $\sigma$ be a complex number defined, up to sign, by:

$$
\operatorname{tr}\left(M_{0} M_{x}\right)=2 \cos (\pi \sigma), \quad|\Re \sigma| \leq 1 .
$$

Actually, $\pm \sigma / 2$ are the eigenvalues of $\lim _{x \rightarrow 0}\left(A_{0}+A_{x}\right)$. The matching procedure yields the following result.

Theorem 2 Let $r \in \mathbf{C}$ and $\sigma$ be as above, with the restriction $|\Re \sigma|<1$. (PVI) has a family of solutions depending on the two parameters $r, \sigma$. The leading terms of the critical behavior for $x \rightarrow 0$ may be parametrized as follows:

For $\sigma \neq 0$ :

$$
y(x) \sim \begin{cases}\frac{1}{r} \frac{\left[\sigma^{2}-\left(\theta_{0}+\theta_{x}\right)^{2}\right]\left[\left(\theta_{0}-\theta_{x}\right)^{2}-\sigma^{2}\right]}{16 \sigma^{3}} x^{1-\sigma}, & \text { if } \Re \sigma>0 ; \\ -\frac{r}{\sigma} x^{1+\sigma}, & \text { if } \Re \sigma<0 ; \\ x\left\{i A \sin (i \sigma \ln x+\phi)+\frac{\theta_{0}^{2}-\theta_{x}^{2}+\sigma^{2}}{2 \sigma^{2}}\right\}, & \text { if } \Re \sigma=0 .\end{cases}
$$

In the above formulae, $r \neq 0$ and

$$
\phi:=i \ln \frac{2 r}{\sigma A}, \quad A:=\left[\frac{\theta_{0}^{2}}{\sigma^{2}}-\left(\frac{\theta_{0}^{2}-\theta_{x}^{2}+\sigma^{2}}{2 \sigma^{2}}\right)^{2}\right]^{\frac{1}{2}}
$$

For special values of $\sigma \neq 0$ :

$$
\begin{aligned}
& y(x) \sim \frac{\theta_{0}}{\theta_{0}+\theta_{x}} x \mp \frac{r}{\theta_{0}+\theta_{x}} x^{1+\sigma}, \quad \sigma= \pm\left(\theta_{0}+\theta_{x}\right) \neq 0, \\
& y(x) \sim \frac{\theta_{0}}{\theta_{0}-\theta_{x}} x \mp \frac{r}{\theta_{0}-\theta_{x}} x^{1+\sigma}, \quad \sigma= \pm\left(\theta_{0}-\theta_{x}\right) \neq 0 .
\end{aligned}
$$

For $\sigma=0$ :

$$
y(x) \sim\left\{\begin{array}{cc}
x\left\{\frac{\theta_{x}^{2}-\theta_{0}^{2}}{4}\left[\ln x+\frac{4 r+2 \theta_{0}}{\theta_{0}^{2}-\theta_{x}^{2}}\right]^{2}+\frac{\theta_{0}^{2}}{\theta_{0}^{2}-\theta_{x}^{2}}\right\}, & \theta_{0} \neq \pm \theta_{x} \\
x\left(r \pm \theta_{0} \ln x\right), & \theta_{0}= \pm \theta_{x}
\end{array}\right.
$$

\section{Comments:}

1) $r$ can be computed as a function of the monodromy data. See (37) and comments there. The sign (branch) of the two quare roots appearing in $\phi$ and $A$ is the same. $x \rightarrow 0$ in a sector of width less then $2 \pi$.

\section{2) Sub-cases of theorem 2 .}

i) When $\sigma \neq 0$, the result of the theorem includes the sub-cases (28) and (29). If $r=0, \theta_{0} \neq 0$, $\theta_{0} \pm \theta_{x} \notin \mathbf{Z}$, direct substitution into (PVI) gives the two Taylor expansions (23). 
If $r \neq 0,(28)$ and (29) are a 1-parameter family, with the restriction $|\Re \sigma|<1$. The symmetry (22) transforms them into the solutions (26), the leading terms being respectively:

$$
\begin{array}{ll}
y(x) \sim \frac{\theta_{\infty}+\theta_{1}-1}{\theta_{\infty}-1}\left(1 \pm \frac{r}{\theta_{\infty}-1} x^{\omega}\right), & \omega= \pm\left(\theta_{\infty}+\theta_{1}-1\right) \neq 0, \\
y(x) \sim \frac{\theta_{\infty}-\theta_{1}-1}{\theta_{\infty}-1}\left(1 \pm \frac{r}{\theta_{\infty}-1} x^{\omega}\right), & \omega= \pm\left(\theta_{\infty}-\theta_{1}-1\right) \neq 0,
\end{array}
$$

with the restriction $|\Re \omega|<1$.

ii) The case $\sigma=0$ includes the sub-case $y(x) \sim r x$, which occurs for $\theta_{0}=\theta_{x}, \theta_{0}=0$. By direct substitution in (PVI) we obtain a series:

$$
y(x)=r x+\sum_{n=3}^{\infty} b_{n}\left(r, \theta_{1}, \theta_{\infty}\right) x^{n}, \quad \theta_{0}=\theta_{x}=0, \quad r \neq 0,1 .
$$

This is again solution (25). Note that for $r=0,1$ we have the singular solutions $y=0, y=1$. Note also that the special sub-sub-case $\theta_{0}=\theta_{x}=\theta_{1}=0$ has applications in the theory of semi-simple Frobenius manifolds of dimension three [7] [10].

3) The first two behaviors (27) were derived in [17]. The existence of such solutions was proved by assuming that the matrices $A_{0}, A_{x}, A_{1}$ have a certain critical behavior for $x \rightarrow 0$, and proving that such matrices solve the Schlesinger equations. Then, the monodromy data were computed by coalescence of singularity. These solutions where further studied in [8], [9], [11], [5]. We show that these solutions can be obtained without any assumption by the matching procedure, together with the solutions (30) and the first of (27), which do not appear in [17].

4) The class of solutions (27) was enlarged in [26] and [11], to the values $\sigma \in \mathbf{C}, \sigma \notin(-\infty, 0] \cup[1,+\infty)$. When $\Re \sigma \geq 1$ or $\Re \sigma \leq 0$, the critical behavior is like the first of (27), and it holds for $x \rightarrow 0$ in a spiral-shaped domain in the universal covering of a punctured neighborhood of $x=0$, along a paths joining a point $x_{0}$ to $x=0$. Along special paths which approach the movable poles, these solution may have behavior $y(x) \sim \sin ^{-2}\left(\frac{i \sigma}{2} \ln x+\varphi(x, r)\right)$, where $\varphi(x, r)$ is a phase depending on the parameter $r$. The transformation $\sigma \mapsto \pm \sigma+2 N, N \in \mathbf{Z}$, leaves the identity $\operatorname{tr}\left(M_{0} M_{x}\right)=2 \cos (\pi \sigma)$ invariant. Its effect on the solutions is studied in [11]. As a result, one can reduce to the values $0 \leq \Re \sigma \leq 1, \sigma \neq 0,1$. We cannot enter into more details here. The reader may find a synthetic description of these results in the review paper [12].

5) Solutions with expansion:

$$
y(x)=x\left(A_{1}+B_{1} \ln x+C_{1} \ln ^{2} x+D_{1} \ln ^{3} x+\ldots\right)+x^{2}\left(A_{2}+B_{2} \ln x+\ldots\right)+\ldots, \quad x \rightarrow 0 .
$$

are all included it theorems 1 and 2. Actually, only the following cases are possible:

$$
y(x)=\left\{\begin{array}{c}
\frac{\theta_{0}}{\theta_{0} \pm \theta_{x}} x+O\left(x^{2}\right) \quad \text { Taylor expansion] } \\
x\left(\frac{\theta_{0}^{2}-B_{1}^{2}}{\theta_{0}^{2}-\theta_{x}^{2}}+B_{1} \ln x+\frac{\theta_{x}^{2}-\theta_{0}^{2}}{4} \ln ^{2} x\right)+x^{2}(\ldots)+\ldots, \\
x\left(A_{1} \pm \theta_{0} \ln x\right)+x^{2}(\ldots)+\ldots, \quad \text { and } \theta_{0}= \pm \theta_{x} .
\end{array}\right.
$$

$A_{1}$ and $B_{1}$ are parameters. We see that the higher orders in (30) are $O\left(x^{2} \ln ^{m} x\right)$, for some integer $m>0$.

6) The symmetry (22) applied to solutions (30) gives:

$$
y(x) \sim 4\left(\left(\theta_{1}^{2}-\left(\theta_{\infty}-1\right)^{2}\right)\left[\ln x-\frac{4 r+2\left(\theta_{\infty}-1\right)}{\theta_{1}^{2}-\left(\theta_{\infty}-1\right)^{2}}\right]^{2}-\frac{4\left(\theta_{\infty}-1\right)^{2}}{\theta_{1}^{2}-\left(\theta_{\infty}-1\right)^{2}}\right)^{-1},
$$

namely:

$$
y(x)=\frac{4}{\left[\theta_{1}^{2}-\left(\theta_{\infty}-1\right)^{2}\right] \ln ^{2} x}\left[1+\frac{8 r+4\left(\theta_{\infty}-1\right)}{\theta_{1}^{2}-\left(\theta_{\infty}-1\right)^{2}} \frac{1}{\ln x}+O\left(\frac{1}{\ln ^{2} x}\right)\right],
$$

and

$$
y(x)=\frac{ \pm 1}{\left(\theta_{\infty}-1\right) \ln x}\left[1 \mp \frac{r}{\left(\theta_{\infty}-1\right) \ln x}+O\left(\frac{1}{\ln ^{2} x}\right)\right], \quad \theta_{\infty} \mp \theta_{1}=1 .
$$


The higer orders $O\left(1 / \ln ^{2} x\right)$ include powers $x^{n}(\ln x)^{ \pm m}$. The so called Chazy solutions, studied in [21] for the special case $\theta_{0}=\theta_{x}=\theta_{1}=0, \theta_{\infty}=-1$, have the behavior (32).

7) When this paper was completed, I received a communication by the first author of [6]. In [6] it is proved that (PVI) has solutions with expansion at $x=\infty$, or $x=0$, of the form $y=c_{r} x^{r}+\sum_{s} c_{s} x^{s}$, $c_{r} \in \mathbf{C}$. The $c_{s}$ 's are either complex constants or polynomials in $\ln x . r$ and $s$ are integer or complex. If $r$ is complex, the restriction $\Re r \in(0,1)$ holds. The method used in [6] is a power geometry technique. The connection problem and the characterization of the associated monodromy data are not studied.

8) When this paper was already accepted for publication, I received a private communication about a recent work [25], on the asymptotics of the real solutions of (PVI). The asymptotic behaviors obtained in [25] are of the type of our theorem 2, namely (27) and the first behavior in (30). The tool used is a method of successive approximations. So, the results are local, and the connection problem is not studied. Moreover, some genericity conditions on the coefficients of PVI seem to be necessary (so, for example, the second solution in (30) cannot be obtained).

\subsection{Monodromy: Result R4).}

In this paper, we computed the monodromy for the Taylor-expanded solutions, which correspond to non-fuchsian reductions of system (4). Because of the symmetries of (PVI), we can limit ourselves to the monodromy data for the representative solutions (18), (19) and (20).

Theorem 3 a) Let $\theta_{\kappa} \notin \mathbf{Z}, \kappa=0,1, x, \infty$. A representation for the monodromy matrices of the solution (18) is:

$$
\begin{gathered}
M_{0}=C_{0 \infty} \exp \left\{i \pi \theta_{0} \sigma_{3}\right\} C_{0 \infty}^{-1}, \\
M_{x}=C_{0 \infty} C_{01}^{-1} \exp \left\{i \pi \theta_{x} \sigma_{3}\right\} C_{01} C_{0 \infty}^{-1} . \\
M_{1}=\exp \left\{-i \pi \theta_{1} \sigma_{3}\right\}, \quad M_{\infty}=\exp \left\{-i \pi \theta_{\infty} \sigma_{3}\right\} .
\end{gathered}
$$

The matrices $C_{0 \infty}$ and $C_{01}$ are:

$$
\begin{gathered}
C_{0 \infty}:=\left[\begin{array}{cc}
\frac{\Gamma\left(1+\frac{\theta_{1}}{2}-\frac{\theta_{\infty}}{2}\right) \Gamma\left(1+\theta_{0}\right) e^{i \frac{\pi}{2}\left[\theta_{0}+\theta_{x}+\theta_{\infty}-\theta_{1}\right]}}{\Gamma\left(\frac{\theta_{0}}{2}+\frac{\theta_{x}}{2}+\frac{\theta_{1}}{2}-\frac{\theta_{\infty}}{2}+1\right) \Gamma\left(\frac{\theta_{0}}{2}-\frac{\theta_{x}}{2}+\frac{\theta_{1}}{2}-\frac{\theta_{\infty}}{2}+1\right)} & \frac{\Gamma\left(1+\frac{\theta_{1}}{2}-\frac{\theta_{\infty}}{2}\right) \Gamma\left(1-\theta_{0}\right) e^{i \frac{\pi}{2}\left[\theta_{x}-\theta_{0}+\theta_{\infty}-\theta_{1}\right]}}{\Gamma\left(-\frac{\theta_{0}}{2}-\frac{\theta_{x}}{2}-\frac{\theta_{\infty}}{2}+\frac{\theta_{1}}{2}+1\right) \Gamma\left(\frac{\theta_{x}}{2}-\frac{\theta_{0}}{2}+\frac{\theta_{1}}{2}-\frac{\theta_{\infty}}{2}+1\right)} \\
-\frac{\Gamma\left(\frac{\theta_{\infty}}{2}-\frac{\theta_{1}}{2}-1\right) \Gamma\left(1+\theta_{0}\right) e^{i \frac{\pi}{2}\left[\theta_{0}+\theta_{x}+\theta_{1}-\theta_{\infty}\right]}}{\Gamma\left(\frac{\theta_{0}}{2}+\frac{\theta_{x}}{2}+\frac{\theta_{\infty}}{2}-\frac{\theta_{1}}{2}\right) \Gamma\left(\frac{\theta_{0}}{2}-\frac{\theta_{x}}{2}+\frac{\theta_{\infty}}{2}-\frac{\theta_{1}}{2}\right)} & -\frac{\Gamma\left(\frac{\theta_{\infty}}{2}-\frac{\theta_{1}}{2}-1\right) \Gamma\left(1-\theta_{0}\right) e^{i \frac{\pi}{2}\left[\theta_{x}-\theta_{0}+\theta_{1}-\theta_{\infty}\right]}}{\Gamma\left(-\frac{\theta_{0}}{2}-\frac{\theta_{x}}{2}-\frac{\theta_{1}}{2}+\frac{\theta_{\infty}}{2}\right) \Gamma\left(\frac{\theta_{x}}{2}-\frac{\theta_{0}}{2}+\frac{\theta_{\infty}}{2}-\frac{\theta_{1}}{2}\right)}
\end{array}\right], \\
C_{01}:=\left[\begin{array}{cc}
\frac{\Gamma\left(-\theta_{x}\right) \Gamma\left(1+\theta_{0}\right)}{\Gamma\left(\frac{\theta_{0}}{2}-\frac{\theta_{x}}{2}+\frac{\theta_{1}}{2}-\frac{\theta_{\infty}}{2}+1\right) \Gamma\left(\frac{\theta_{0}}{2}-\frac{\theta_{x}}{2}+\frac{\theta_{\infty}}{2}-\frac{\theta_{1}}{2}\right)} & \frac{\Gamma\left(-\theta_{x}\right) \Gamma\left(1-\theta_{0}\right)}{\Gamma\left(-\frac{\theta_{0}}{2}-\frac{\theta_{x}}{2}-\frac{\theta_{\infty}}{2}+\frac{\theta_{1}}{2}+1\right) \Gamma\left(-\frac{\theta_{0}}{2}-\frac{\theta_{x}}{2}-\frac{\theta_{1}}{2}+\frac{\theta_{\infty}}{2}\right)} \\
\frac{\Gamma\left(\theta_{x}\right) \Gamma\left(1+\theta_{0}\right)}{\Gamma\left(\frac{\theta_{0}}{2}+\frac{\theta_{x}}{2}+\frac{\theta_{\infty}}{2}-\frac{\theta_{1}}{2}\right) \Gamma\left(\frac{\theta_{0}}{2}+\frac{\theta_{x}}{2}+\frac{\theta_{1}}{2}-\frac{\theta_{\infty}}{2}+1\right)} & \left.\frac{\Gamma\left(\frac{\theta_{x}}{2}-\frac{\theta_{0}}{2}+\frac{\theta_{\infty}}{2}-\frac{\theta_{x}}{2}\right) \Gamma\left(1-\theta_{0}\right)}{2}-\frac{\theta_{0}}{2}+\frac{\theta_{1}}{2}-\frac{\theta_{\infty}}{2}+1\right)
\end{array}\right],
\end{gathered}
$$

The subgroup generated by $M_{0} M_{x}$ and $M_{1}$ is reducible. As for the solution (21), we just need to change $\theta_{1} \mapsto-\theta_{1}$.

b) It is convenient to re-parameterize the solution (19) by introducing a parameter s through the equality:

$$
a=\frac{\theta_{\infty}\left(2 s+\theta_{x}+1\right)}{2\left(\theta_{\infty}-1\right)} .
$$

Let $\theta_{x}, \theta_{\infty} \notin \mathbf{Z}$. Then, a representation for the monodromy group is:

$$
\begin{array}{cc}
M_{0}=G \exp \left\{i \pi \theta_{x} \sigma_{3}\right\} G^{-1}, & M_{1}=\exp \left\{-i \pi \theta_{\infty} \sigma_{3}\right\} \\
M_{x}=G \exp \left\{-i \pi \theta_{x} \sigma_{3}\right\} G^{-1}, & M_{\infty}=\exp \left\{-i \pi \theta_{\infty} \sigma_{3}\right\}
\end{array}
$$


In particular, $M_{1}=M_{\infty}, M_{0} M_{x}=I$. We can choose $G$ as follows:

$$
G=\left(\begin{array}{cc}
1 & 1 \\
\frac{s+\theta_{x}}{r} & \frac{s}{r}
\end{array}\right) .
$$

Conversely, we may express $s$ as a funcition of the monodromy data:

$$
s=\frac{\theta_{x}\left[2 \cos \left(\pi\left(\theta_{\infty}+\theta_{x}\right)\right)-\operatorname{tr}\left(M_{1} M_{0}\right)\right]}{2\left[\cos \left(\pi\left(\theta_{\infty}-\theta_{x}\right)\right)-\cos \left(\pi\left(\theta_{\infty}+\theta_{x}\right)\right)\right]} .
$$

c) We re-parameterize solution (20) introducing a new parameter $s$ defined by $a=:(1-s)^{-1}$. Let $\theta_{0}, \theta_{x} \notin \mathbf{Z}$. Then, a monodromy representation for the solutions (20) is:

$$
\begin{aligned}
& M_{0}=\left(C_{\infty 0}\right)^{-1} \exp \left\{i \pi \theta_{0} \sigma_{3}\right\} C_{\infty 0}, \quad M_{\infty}=\left(\begin{array}{cc}
-1 & 0 \\
2 \pi i(1-s) & -1
\end{array}\right) \\
& M_{x}=\left(C_{\infty 0}\right)^{-1}\left(C_{01}\right)^{-1} \exp \left\{i \pi \theta_{x} \sigma_{3}\right\} C_{01} C_{\infty 0}, \quad M_{1}=\left(\begin{array}{cc}
1 & 0 \\
2 \pi i s & 1
\end{array}\right) .
\end{aligned}
$$

where $C_{\infty 0}$ and $C_{01}$ are (35) and (36) given below. Conversely, we may express $s$ as a function of the monodromy data:

$$
s=\frac{\operatorname{tr}\left(M_{1} M_{0}\right)-2 \cos \left(\pi \theta_{0}\right)}{4 \pi \sin \left(\pi \theta_{0}\right)} \frac{\left(C_{\infty 0}\right)_{21}}{\left(C_{\infty 0}\right)_{22}} .
$$

The matrices $C_{\infty 0}$ and $C_{01}$ are:

$$
\begin{aligned}
& C_{\infty 0}=2\left(\begin{array}{cc}
0 & \frac{\Gamma\left(-\theta_{0}\right) e^{-i \pi\left\{\frac{\theta_{0}}{2}+\frac{\theta_{x}}{2}+\frac{3}{2}\right\}}}{\Gamma\left(-\frac{\theta_{0}}{2}-\frac{\theta_{x}}{2}+\frac{3}{2}\right) \Gamma\left(-\frac{\theta_{0}}{2}+\frac{\theta_{x}}{2}+\frac{3}{2}\right)} \\
-\frac{\Gamma\left(-\frac{\theta_{0}}{2}-\frac{\theta_{x}}{2}-\frac{1}{2}\right) \Gamma\left(-\frac{\theta_{0}}{2}+\frac{\theta_{x}}{2}-\frac{1}{2}\right)}{\Gamma\left(1-\theta_{0}\right) e^{-i \pi\left\{\frac{\theta_{0}}{2}-\frac{\theta_{x}}{2}-\frac{3}{2}\right\}}} & \frac{\Gamma\left(\theta_{0}\right) e^{-i \pi\left\{-\frac{\theta_{0}}{2}+\frac{\theta_{x}}{2}+\frac{3}{2}\right\}}}{\Gamma\left(\frac{\theta_{0}}{2}-\frac{\theta_{x}}{2}+\frac{3}{2}\right) \Gamma\left(\frac{\theta_{0}}{2}+\frac{\theta_{x}}{2}+\frac{3}{2}\right)}
\end{array}\right) . \\
& C_{01}=\left(\begin{array}{cc}
\frac{\Gamma\left(-\theta_{x}\right) \Gamma\left(1+\theta_{0}\right)}{\Gamma\left(\frac{\theta_{0}}{2}-\frac{\theta_{x}}{2}+\frac{3}{2}\right) \Gamma\left(\frac{\theta_{0}}{2}-\frac{\theta_{x}}{2}-\frac{1}{2}\right)} & \frac{\Gamma\left(-\theta_{x}\right) \Gamma\left(1-\theta_{0}\right)}{\Gamma\left(-\frac{\theta_{0}}{2}-\frac{\theta_{x}}{2}+\frac{3}{2}\right) \Gamma\left(-\frac{\theta_{0}}{2}-\frac{\theta_{x}}{2}-\frac{1}{2}\right)} \\
\frac{\Gamma\left(\theta_{x}\right) \Gamma\left(1+\theta_{0}\right)}{\Gamma\left(\frac{\theta_{0}}{2}+\frac{\theta_{x}}{2}+\frac{3}{2}\right) \Gamma\left(\frac{\theta_{0}}{2}+\frac{\theta_{x}}{2}+\frac{1}{2}\right)} & \frac{\Gamma\left(\theta_{x}\right) \Gamma\left(1-\theta_{0}\right)}{\Gamma\left(-\frac{\theta_{0}}{2}+\frac{\theta_{x}}{2}+\frac{3}{2}\right) \Gamma\left(-\frac{\theta_{0}}{2}+\frac{\theta_{x}}{2}-\frac{1}{2}\right)}
\end{array}\right)
\end{aligned}
$$

The conditions $\theta_{\kappa} \notin \mathbf{Z}$ can be eliminated, and the computations can be repeated without conceptual changes, but with different results.

In the above theorem, the subgroups generated by $M_{0} M_{x}$ and $M_{1}$ are reducible. This characterizes the monodromy associated to solutions which have a Taylor series at $x=0$. The same characterization at $x=1$ involves the subgroup generated by $M_{1} M_{x}$ and $M_{0}$. At $x=\infty$, it involves the subgroup generated by $M_{0} M_{1}$ and $M_{x} .{ }^{2}$ In another paper, we will consider again this characterization, together with the general problem of classification.

Let us define again $\sigma$ by $\operatorname{tr}\left(M_{0} M_{x}\right)=2 \cos \pi \sigma$. Then, in case a), $\sigma= \pm\left(\theta_{1}-\theta_{\infty}\right)$ [and $\pm\left(\theta_{1}+\theta_{\infty}\right)$ for the change $\left.\theta_{1} \mapsto-\theta_{1}\right]$. In case $\left.\mathrm{b}\right), \operatorname{tr}\left(M_{0} M_{x}\right)=2$ and $\sigma=0$. In case c), $\operatorname{tr}\left(M_{0} M_{x}\right)=-2, \sigma= \pm 1$. The matching procedure is effective to produce solutions corresponding to monodromy data for which the connection problem is so far not well studied, such as the case $\operatorname{tr}\left(M_{i} M_{j}\right)=-2$ (see [12]). ${ }^{3}$

Note: Also the 1-parameter solutions (28) (29) and the second solution in (30) are characterized by a reducible subgroup generated by $M_{0}, M_{x}$.

\footnotetext{
${ }^{2}$ In the appendix of [11], the reader may find explanantions about how to obtain results at $x=1, \infty$ from the results at $x=0$

${ }^{3}$ Here I remark that the formula (1.30), page 1293, of my paper [11] is wrong. The correct one is $\operatorname{tr}\left(M_{i} M_{j}\right) \notin$ $(-\infty,-2]$. In [11] the connection problem is solved for $\operatorname{tr}\left(M_{i} M_{j}\right) \neq \pm 2$. The case $\operatorname{tr}\left(M_{i} M_{j}\right)=2$ yields $(30)$. For the special choice of the parameters $\theta_{0}=\theta_{x}=\theta_{1}=0$, it was studied in [8] and [9] (no logarithmic terms appear in such a special case). The result (30) for the general (PVI), corresponding to $\operatorname{tr}\left(M_{0} M_{x}\right)=2$, appears in the present paper for the first time.
} 


\section{Comments.}

1) The monodromy group for the solutions (23) was derived also in [19], by confluence of singularities of scalar equations (including a Heun's type equation). The result is equivalent to that in point a) of the above theorem.

2) The computation of the monodromy group of the fuchsian systems (13) and (14) is quite clear [17] [8] [11] [5]. It allows to express the parameter $r$ of (27), (28), (29) and (30) as a function of the monodromy data. Though the computation for (28), (29) and (30) does not appear in the literature, the procedure is clear (see section 4.8), so we do not repeat it. We just report the result for (27), which can be found in [17] [11] [5]:

$$
r=\frac{\left(\theta_{0}-\theta_{x}+\sigma\right)\left(\theta_{0}+\theta_{x}-\sigma\right)\left(\theta_{\infty}+\theta_{1}-\sigma\right)}{4 \sigma\left(\theta_{\infty}+\theta_{1}+\sigma\right)} \frac{1}{\mathbf{F}}
$$

where

$$
\begin{aligned}
\mathbf{F}:= & \frac{\Gamma(1+\sigma)^{2} \Gamma\left(\frac{1}{2}\left(\theta_{0}+\theta_{x}-\sigma\right)+1\right) \Gamma\left(\frac{1}{2}\left(\theta_{x}-\theta_{0}-\sigma\right)+1\right)}{\Gamma(1-\sigma)^{2} \Gamma\left(\frac{1}{2}\left(\theta_{0}+\theta_{x}+\sigma\right)+1\right) \Gamma\left(\frac{1}{2}\left(\theta_{x}-\theta_{0}+\sigma\right)+1\right)} \times \\
& \times \frac{\Gamma\left(\frac{1}{2}\left(\theta_{\infty}+\theta_{1}-\sigma\right)+1\right) \Gamma\left(\frac{1}{2}\left(\theta_{1}-\theta_{\infty}-\sigma\right)+1\right)}{\Gamma\left(\frac{1}{2}\left(\theta_{\infty}+\theta_{1}+\sigma\right)+1\right) \Gamma\left(\frac{1}{2}\left(\theta_{1}-\theta_{\infty}+\sigma\right)+1\right)} \frac{V}{U}
\end{aligned}
$$

and:

$$
\begin{aligned}
& U:= {\left[\frac{i}{2} \sin (\pi \sigma) \operatorname{tr}\left(M_{1} M_{x}\right)-\cos \left(\pi \theta_{x}\right) \cos \left(\pi \theta_{\infty}\right)-\cos \left(\pi \theta_{0}\right) \cos \left(\pi \theta_{1}\right)\right] e^{i \pi \sigma}+} \\
&+\frac{i}{2} \sin (\pi \sigma) \operatorname{tr}\left(M_{0} M_{1}\right)+\cos \left(\pi \theta_{x}\right) \cos \left(\pi \theta_{1}\right)+\cos \left(\pi \theta_{\infty}\right) \cos \left(\pi \theta_{0}\right) \\
& V:=4 \sin \frac{\pi}{2}\left(\theta_{0}+\theta_{x}-\sigma\right) \sin \frac{\pi}{2}\left(\theta_{0}-\theta_{x}+\sigma\right) \sin \frac{\pi}{2}\left(\theta_{\infty}+\theta_{1}-\sigma\right) \sin \frac{\pi}{2}\left(\theta_{\infty}-\theta_{1}+\sigma\right) .
\end{aligned}
$$

The above formula was computed with the assumption that $\sigma \pm\left(\theta_{0}+\theta_{x}\right), \sigma \pm\left(\theta_{0}-\theta_{x}\right), \sigma \pm\left(\theta_{1}+\theta_{\infty}\right)$, $\sigma \pm\left(\theta_{1}-\theta_{\infty}\right)$ are not even integers. ${ }^{4}$

3) Reducible Monodromy. The monodromy groups in theorem 3 are not reducible, but they have a reducible subgroup. If the entire group itself is completely reducible, all the Painleveé transcendents are known. Solutions of (PVI) corresponding to a reducible monodromy were found in [13]). We summarize the results:

Proposition 1 All the solutions of (PVI) corresponding to a reducible monodromy group are equivalent by birational canonical transformations to the following one-parameter family of solutions, with $\theta_{\infty}+\theta_{1}+\theta_{0}+\theta_{x}=0$ :

$$
y(x)=\frac{\theta_{1}+\theta_{\infty}-1+x\left(1+\theta_{x}\right)}{\theta_{\infty}-1}-\frac{1}{\theta_{\infty}-1} \frac{x(1-x)}{u(x ; a)} \frac{d u(x ; a)}{d x},
$$

where $u(x ; a)=u_{1}(x)+a u_{2}(x) ; a \in \mathbf{C}, u_{1}(x)$ and $u_{2}(x)$ are linear independent solutions of the hyper-geometric equation:

$$
x(1-x) \frac{d^{2} u}{d x^{2}}+\left\{\left[2-\left(\theta_{\infty}+\theta_{1}\right)\right]-\left(4-\theta_{\infty}+\theta_{x}\right) x\right\} \frac{d u}{d x}-\left(2-\theta_{\infty}\right)\left(1+\theta_{x}\right) u=0
$$

The monodromy matrices are

$$
M_{0}=\left(\begin{array}{cc}
\frac{\theta_{0}}{2} & * \\
0 & -\frac{\theta_{0}}{2}
\end{array}\right), \quad M_{x}=\left(\begin{array}{cc}
\frac{\theta_{x}}{2} & * \\
0 & -\frac{\theta_{x}}{2}
\end{array}\right), \quad M_{1}=\left(\begin{array}{cc}
\frac{\theta_{1}}{2} & * \\
0 & -\frac{\theta_{1}}{2}
\end{array}\right) .
$$

The parameter $a$ does not appear in the monodromy.

\footnotetext{
${ }^{4}$ In [11] there is a missprint in formula (A.30), which must be re-calculated. In [17], in formula (1.8) at the bottom of page 1141, the last sign is $\pm \sigma$ instead of $\mp \sigma$.
} 
Remark: The rational solutions of (PVI) are a special case of the above proposition. They were studied in [22]. Up to canonical birational transformations, they are realized for $\theta_{\infty}+\theta_{1}+\theta_{0}+\theta_{x}=0$ and:

$$
\begin{gathered}
\theta_{0}=1: \quad y(x)=\frac{\theta_{\infty}+\theta_{1}}{\theta_{\infty}} \frac{x-1}{x\left(1+\theta_{1}\right)-\left(\theta_{1}+\theta_{\infty}\right)} \\
\theta_{0}=-2: \quad y(x)=\frac{\left(2-\left(\theta_{\infty}+\theta_{1}\right)+\theta_{1} x\right)^{2}-2+\theta_{\infty}+\theta_{1}-\theta_{1} x^{2}}{\left(1-\theta_{\infty}\right)\left(2-\left(\theta_{\infty}+\theta_{1}\right)+\theta_{1} x\right)} .
\end{gathered}
$$

The computation of the expansion at $x=0$ of (38) is just a consequence of the expansions of $u_{1}(x)$ and $u_{2}(x)$. The reader can find by himself a behavior $y \sim x\left(r(a) \pm \theta_{x} \ln (x)\right)$ for $\theta_{1}+\theta_{\infty}=\theta_{0}+\theta_{x}=0$, namely a sub-case of the second solution in (30). For $\theta_{1}+\theta_{\infty} \notin \mathbf{Z}$, we find behaviors of the type (26) (and (18), (23) for $a=0$ ).

\section{PART II - Derivation Results: Fuchsian Reduction}

\section{Fuchsian Case}

Let $x \rightarrow 0$. The reduction to the fuchsian systems (13) is possible if in the domain (7) we have:

$$
\left|\left(A_{0}+A_{x}\right)_{i j}\right| \gg\left|\left(A_{x}\right)_{i j} \frac{x}{\lambda}\right|, \quad \text { namely: }\left|\left(A_{0}+A_{x}\right)_{i j}\right| \gg\left|\left(A_{x}\right)_{i j} x^{1-\delta_{O U T}}\right| .
$$

Let us denote with $\hat{A}_{i}$ the leading term of the matrix $A_{i}, i=0, x, 1$. We can substitute (13) with:

$$
\frac{d \Psi_{\text {OUT }}}{d \lambda}=\left[\frac{\hat{A}_{0}+\hat{A}_{x}}{\lambda}+\frac{\hat{A}_{1}}{\lambda-1}\right] \Psi_{\text {OUT }}
$$

We suppose that $\theta_{\infty} \neq 0$. This is not a loss in generality, because $\theta_{\infty}=0$ is equivalent to $\theta_{\infty}=2$.

Lemma 1 If the approximation (13) is possible, then $\hat{A}_{0}+\hat{A}_{x}$ has eigenvalues $\pm \frac{\sigma}{2} \in \mathbf{C}$ independent of $x$, defined (up to sign and addition of an integer) by $\operatorname{tr}\left(M_{x} M_{0}\right)=2 \cos (\pi \sigma)$. Let $r_{1} \in \mathbf{C}, r_{1} \neq 0$. For $\theta_{\infty} \neq 0$, the leading terms are:

$$
\hat{A_{1}}=\left(\begin{array}{cc}
\frac{\sigma^{2}-\theta_{\infty}^{2}-\theta_{1}^{2}}{4 \theta_{\infty}} & -r_{1} \\
\frac{\left[\sigma^{2}-\left(\theta_{1}-\theta_{\infty}\right)^{2}\right]\left[\sigma^{2}-\left(\theta_{1}+\theta_{\infty}\right)^{2}\right]}{16 \theta_{\infty}^{2}} \frac{1}{r_{1}} & -\frac{\sigma^{2}-\theta_{\infty}^{2}-\theta_{1}^{2}}{4 \theta_{\infty}}
\end{array}\right),
$$

and

$$
\hat{A_{0}}+\hat{A_{x}}=\left(\begin{array}{cc}
\frac{\theta_{1}^{2}-\sigma^{2}-\theta_{\infty}^{2}}{4 \theta_{\infty}} & r_{1} \\
-\frac{\left[\sigma^{2}-\left(\theta_{1}-\theta_{\infty}\right)^{2}\right]\left[\sigma^{2}-\left(\theta_{1}+\theta_{\infty}\right)^{2}\right]}{16 \theta_{\infty}^{2}} \frac{1}{r_{1}} & -\frac{\theta_{1}^{2}-\sigma^{2}-\theta_{\infty}^{2}}{4 \theta_{\infty}}
\end{array}\right) .
$$

Proof: Observe that $\operatorname{tr}\left(\hat{A}_{0}+\hat{A}_{x}\right)=\operatorname{tr}\left(A_{0}+A_{x}\right)=0$, thus, for any $x, \hat{A}_{0}+\hat{A}_{x}$ has eigenvalues of opposite sign, that we denote $\pm \tilde{\sigma}(x) / 2$. Then, we recall that $x$ is a monodromy preserving deformation, therefore the monodromy matrices of (40) are independent of $x$. At $\lambda=0,1, \infty$ they are:

$$
M_{0}^{\text {OUT }}=\left\{\begin{array}{l}
M_{x} M_{0} \\
M_{0} M_{x}
\end{array}, \quad M_{1}^{\text {OUT }}=M_{1}, \quad M_{\infty}^{\text {OUT }}=M_{\infty} .\right.
$$

Thus, $\operatorname{det}\left(M_{0}^{\text {OUT }}\right)=1$, because $\operatorname{det}\left(M_{x}\right)=\operatorname{det}\left(M_{0}\right)=1$. Therefore, there exists a constant matrix $D$ and a complex constant number $\sigma$ such that:

$$
D^{-1} M_{0}^{\text {OUT }} D=\left\{\begin{array}{c}
\operatorname{diag}(\exp \{-i \pi \sigma\}, \exp \{i \pi \sigma\}), \\
\left(\begin{array}{cc} 
\pm 1 & * \\
0 & \pm 1
\end{array}\right), \text { or }\left(\begin{array}{cc} 
\pm 1 & 0 \\
* & \pm 1
\end{array}\right), \quad \sigma \in \mathbf{Z}
\end{array}\right.
$$


We conclude that $\tilde{\sigma}(x) \equiv \sigma$. We also have $\operatorname{tr}\left(M_{0}^{O U T}\right)=2 \cos (\pi \sigma)$.

Now consider the gauge:

$$
\Phi_{1}:=\lambda^{-\frac{\sigma}{2}}(\lambda-1)^{-\frac{\theta_{1}}{2}} \Psi_{\text {OUT }} . \quad \frac{d \Phi_{1}}{d \lambda}=\left[\frac{\hat{A}_{0}+\hat{A}_{x}-\frac{\sigma}{2}}{\lambda}+\frac{\hat{A}_{1}-\frac{\theta_{1}}{2}}{\lambda-1}\right] \Phi_{1}
$$

We can identify $\hat{A}_{0}+\hat{A}_{x}-\frac{\sigma}{2}$ and $\hat{A}_{1}-\frac{\theta_{1}}{2}$ with $B_{0}$ and $B_{1}$ of Proposition 2 in Appendix 1 , case (98), with $a=\frac{\theta_{\infty}}{2}+\frac{\theta_{1}}{2}+\frac{\sigma}{2}, b=-\frac{\theta_{\infty}}{2}+\frac{\theta_{1}}{2}+\frac{\sigma}{2}, c=\sigma$.

Remark: $r_{1}$ may be a function of $x$. If the monodromy of system (40) depends on $r_{1}$, then $r_{1}$ is a constant independent of $x$. This is the case here, but we do not prove it for reasons of space. See the references in Part I.

Lemma 1 (and Lemma 3 which follows) includes all cases (98)-(102) for system (43). Cases (99) - (102) are obtained substituting $\sigma=-\left(\theta_{\infty}+\theta_{1}\right), \theta_{\infty}-\theta_{1}, \theta_{\infty}+\theta_{1}, \theta_{1}-\theta_{\infty}$ respectively. For all the computations which follow, involving system (40) or (43), we note that the hypothesis $\theta_{\infty} \neq 0$ excludes cases (103), (104) and the Jordan cases (105)-(107).

The reduction to the fuchsian system (14) is possible for $x \rightarrow 0$ in the domain (9) if:

$$
\left|\frac{\left(A_{0}\right)_{i j}}{\lambda}+\frac{\left(A_{x}\right)_{i j}}{\lambda-x}\right| \gg\left|\left(A_{1}\right)_{i j}\right|, \quad \text { namely: }\left|\frac{\left(A_{0}+A_{x}\right)_{i j}}{x^{\delta_{I N}}}\right| \gg\left|\left(A_{1}\right)_{i j}\right| .
$$

We can rewrite (14) using just the leading terms of the matrices:

$$
\frac{d \Psi_{I N}}{d \lambda}=\left[\frac{\hat{A_{0}}}{\lambda}+\frac{\hat{A_{x}}}{\lambda-x}\right] \Psi_{I N}
$$

Then, we re-scale $\lambda$ and consider the following system:

$$
\frac{d \Psi_{I N}}{d \mu}=\left(\frac{\hat{A}_{0}}{\mu}+\frac{\hat{A}_{x}}{\mu-1}\right) \Psi_{I N}, \quad \mu:=\frac{\lambda}{x}
$$

We know that there exists a matrix $K_{0}(x)$ such that:

$$
K_{0}^{-1}(x)\left(\hat{A}_{0}+\hat{A}_{x}\right) K_{0}(x)=\left(\begin{array}{cc}
\frac{\sigma}{2} & 0 \\
0 & -\frac{\sigma}{2}
\end{array}\right), \quad \text { or } \quad\left(\begin{array}{ll}
0 & 1 \\
0 & 0
\end{array}\right) .
$$

Let $\hat{\hat{A}}_{i}:=K_{0}{ }^{-1} \hat{A}_{i} K_{0}, i=0, x$. By a gauge transformation, we get the system:

$$
\Psi_{I N}=: K_{0}(x) \Psi_{0}, \quad \frac{d \Psi_{0}}{d \mu}=\left[\frac{\hat{\hat{A}_{0}}}{\mu}+\frac{\hat{\hat{A}}}{\mu-1}\right] \Psi_{0},
$$

Lemma 2 Let $r \in \mathbf{C}, r \neq 0$. If $\sigma \neq 0$, we have:

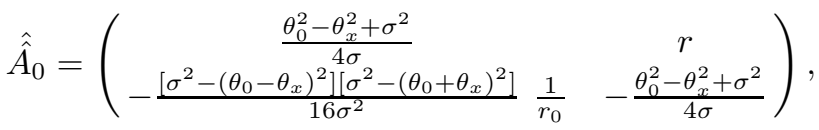

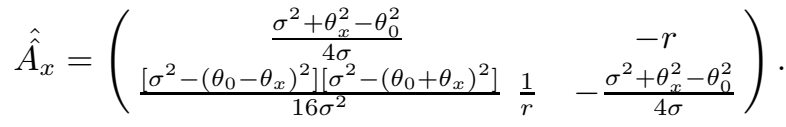

Proof: We do a gauge transformation:

$$
\Phi_{0}:=\mu^{-\frac{\theta_{0}}{2}}(\mu-1)^{-\frac{\theta_{x}}{2}} \Psi_{0}, \quad \frac{d \Phi_{0}}{d \mu}=\left[\frac{\hat{\hat{\hat{A}_{0}}}-\frac{\theta_{0}}{2}}{\mu}+\frac{\hat{\hat{A}}_{x}-\frac{\theta_{x}}{2}}{\mu-1}\right] \Phi_{0} .
$$


We identify $\hat{\hat{A}}_{0}-\frac{\theta_{0}}{2}, \hat{\hat{A}}_{x}-\frac{\theta_{x}}{2}$ with $B_{0}$ and $B_{1}$ in the Appendix 1, Proposition 2, case (98), with $a=\frac{\theta_{0}}{2}+\frac{\theta_{x}}{2}-\frac{\sigma}{2}, b=\frac{\theta_{0}}{2}+\frac{\theta_{x}}{2}+\frac{\sigma}{2}, c=\theta_{0}$.

Remark: If the monodromy of the system (46) depends on $r$, then $r$ is a constant independent of $x$. This is the case here.

The Lemma 2 (and lemma 4 which follows) includes also the cases (99)-(102) for the system (49). These cases correspond respectively to the values $\sigma=\theta_{0}+\theta_{x},-\theta_{0}-\theta_{x}, \theta_{x}-\theta_{0}, \theta_{0}-\theta_{x}$, with $\theta_{0} \neq \pm \theta_{x}$.

\subsection{Matching for $\sigma \notin \mathrm{Z}$ and proof of (27)}

We match $\Psi_{O U T}$ and $\Psi_{I N}$ in the intersection of the "outside" and "inside" domains, namely the region $|x|^{\delta_{\text {OUT }}} \leq|\lambda| \leq|x|^{\delta_{I N}}, x \rightarrow 0$. As a consequence, we obtain the leading term of $y(x)$.

Lemma 3 If $\sigma \notin \mathbf{Z}$ and $\theta_{\infty} \neq 0$, system (40) has a fundamental matrix solution $\Psi_{O U T}(\lambda)$ with the following behavior at $\lambda=0$ :

$$
\Psi_{\text {OUT }}=\sum_{n=0}^{\infty} G_{n} \lambda^{n}\left(\begin{array}{cc}
\lambda^{\frac{\sigma}{2}} & 0 \\
0 & \lambda^{-\frac{\sigma}{2}}
\end{array}\right), \quad G_{0}=\left(\begin{array}{cc}
1 & 1 \\
\frac{\left(\theta_{\infty}+\sigma\right)^{2}-\theta_{1}^{2}}{4 \theta_{\infty} r_{1}} & \frac{\left(\theta_{\infty}-\sigma\right)^{2}-\theta_{1}^{2}}{4 \theta_{\infty} r_{1}}
\end{array}\right) .
$$

$G_{n}$ are matrices which depend rationally on $\theta_{\infty}, \theta_{1}, \sigma, r_{1}$. The series is convergent for $|\lambda|<1$.

Proof: It is an immediate consequence of the standard theory of linear systems of fuchsian differential equations.

Lemma 4 If $\sigma \notin \mathbf{Z}$, system (46) has a fundamental matrix solution with the following behavior at $\mu=\infty$ :

$$
\Psi_{0}(\mu)=\left[I+\sum_{n=1}^{\infty} K_{n} \mu^{-n}\right]\left(\begin{array}{cc}
\mu^{\frac{\sigma}{2}} & 0 \\
0 & \mu^{-\frac{\sigma}{2}}
\end{array}\right),
$$

where $I$ is the identity matrix, $K_{n}$ are matrices which depend rationally on $\theta_{0}, \theta_{x}, \sigma, r$. The series is convergent for $|\mu|>1$.

Proof: It is a consequence of the standard theory of systems of fuchsian equations.

The matching relation $\Psi_{1}(\lambda) \sim K_{0}(x) \Psi_{0}(\lambda / x),|x|^{\delta_{\text {OUT }}} \leq|\lambda| \leq|x|^{\delta_{I N}}, x \rightarrow 0$, is:

$$
G_{0}\left(\begin{array}{cc}
\lambda^{\frac{\sigma}{2}} & 0 \\
0 & \lambda^{-\frac{\sigma}{2}}
\end{array}\right) \sim K_{0}(x)\left(\begin{array}{cc}
\lambda^{\frac{\sigma}{2}} & 0 \\
0 & \lambda^{-\frac{\sigma}{2}}
\end{array}\right)\left(\begin{array}{cc}
x^{-\frac{\sigma}{2}} & 0 \\
0 & x^{\frac{\sigma}{2}}
\end{array}\right) .
$$

This gives the result:

$$
K_{0}(x) \sim\left(\begin{array}{cc}
1 & 1 \\
\frac{\left(\theta_{\infty}+\sigma\right)^{2}-\theta_{1}^{2}}{4 \theta_{\infty} r_{1}} & \frac{\left(\theta_{\infty}-\sigma\right)^{2}-\theta_{1}^{2}}{4 \theta_{\infty} r_{1}}
\end{array}\right)\left(\begin{array}{cc}
x^{\frac{\sigma}{2}} & 0 \\
0 & x^{-\frac{\sigma}{2}}
\end{array}\right) .
$$

We compute the matrices $\hat{A_{0}}(x)=K_{0}(x) \hat{\hat{A}_{0}} K_{0}(x)^{-1}, \hat{A_{x}}(x)=K_{0}(x) \hat{\hat{A_{x}}} K_{0}(x)^{-1}$ making use of Lemma 2. We obtain:

$$
\begin{aligned}
& \hat{A_{0}}(x)=G_{0}\left(\begin{array}{cc}
\frac{\theta_{0}^{2}-\theta_{x}^{2}+\sigma^{2}}{4 \sigma} & r x^{\sigma} \\
-\frac{\left(\sigma+\theta_{x}-\theta_{0}\right)\left(\sigma+\theta_{x}+\theta_{0}\right)\left(\sigma-\theta_{x}+\theta_{0}\right)\left(\sigma-\theta_{x}-\theta_{0}\right)}{16 \sigma^{2} r} x^{-\sigma} & -\frac{\theta_{0}^{2}-\theta_{x}^{2}+\sigma^{2}}{4 \sigma}
\end{array}\right) G_{0}^{-1} \\
& \hat{A}_{x}(x)=G_{0}\left(\begin{array}{cc}
\frac{\sigma^{2}+\theta_{x}^{2}-\theta_{0}^{2}}{4 \sigma} & -r x^{\sigma} \\
\frac{\left(\sigma+\theta_{x}-\theta_{0}\right)\left(\sigma+\theta_{x}+\theta_{0}\right)\left(\sigma-\theta_{x}+\theta_{0}\right)\left(\sigma-\theta_{x}-\theta_{0}\right)}{16 \sigma^{2} r} x^{-\sigma} & -\frac{\sigma^{2}+\theta_{x}^{2}-\theta_{0}^{2}}{4 \sigma}
\end{array}\right) G_{0}{ }^{-1} .
\end{aligned}
$$

This result shows that the matrix elements of $\hat{A_{0}}$ and $\hat{A_{x}}$ diverge as $|x|^{-|\Re \sigma|}$ when $x \rightarrow 0$ inside a sector (i.e. for $|\arg (x)|$ bounded). In particular, we find $\left(\hat{A_{1}}\right)_{12}=-r_{1}$ and

$$
\left(\hat{A}_{0}\right)_{12}=\frac{r_{1}}{r} \frac{\left[\sigma^{2}-\left(\theta_{0}+\theta_{x}\right)^{2}\right]\left[\left(\theta_{0}-\theta_{x}\right)^{2}-\sigma^{2}\right]}{16 \sigma^{3}} x^{-\sigma}+r_{1} \frac{\theta_{0}^{2}-\theta_{x}^{2}+\sigma^{2}}{2 \sigma^{2}}-\frac{r r_{1}}{\sigma} x^{\sigma} .
$$


The above are enough to compute the leading term(s) of $y(x)$ from the formula:

$$
y(x)=\frac{x\left(A_{0}\right)_{12}}{x\left[\left(A_{0}\right)_{12}+\left(A_{1}\right)_{12}\right]-\left(A_{1}\right)_{12}}=-\frac{x\left(A_{0}\right)_{12}}{\left(A_{1}\right)_{12}}\left[1-x\left(1+\frac{\left(A_{0}\right)_{12}}{\left(A_{1}\right)_{12}}\right)\right]^{-1}
$$

Thus:

$$
y(x) \sim-x \frac{\left(\hat{A}_{0}\right)_{12}}{\left(\hat{A}_{1}\right)_{12}}=\left[\frac{1}{r} \frac{\left[\sigma^{2}-\left(\theta_{0}+\theta_{x}\right)^{2}\right]\left[\left(\theta_{0}-\theta_{x}\right)^{2}-\sigma^{2}\right]}{16 \sigma^{3}} x^{1-\sigma}+\frac{\theta_{0}^{2}-\theta_{x}^{2}+\sigma^{2}}{2 \sigma^{2}} x-\frac{r}{\sigma} x^{1+\sigma}\right] .
$$

We have ignored $1 /\left[1-x\left(1+\left(\hat{A_{0}}\right)_{12} /\left(\hat{A_{1}}\right)_{12}\right)\right]$ because condition (39) is equivalent to: $\left|x^{1-\delta_{1} \pm \sigma}\right| \rightarrow$

0 for $x \rightarrow 0$, which implies that $\left|x\left(\hat{A_{0}}\right)_{12} /\left(\hat{A_{1}}\right)_{12}\right| \sim\left|x^{1 \pm \sigma}\right| \rightarrow 0$. Therefore, $1 /[1-x(1+$ $\left.\left.\left(\hat{A}_{0}\right)_{12} /\left(\hat{A}_{1}\right)_{12}\right)\right]=1 /(1+O(x))=1+O(x)$.

If $\Re \sigma \neq 0$, the leading term of (51) is certainly correct, but some higher order corrections may be bigger than the next two terms of (51). If $\Re \sigma=0$, the three terms of (51) are of the same order, and their combination gives the trigonometric expression in theorem 2.

\subsection{Range of $\sigma$}

Conditions (39) and (44) must be verified. Let $C$ denote a non zero constant. We suppose that $x \rightarrow 0$ inside a sector with center on $x=0$. Then:

$$
\begin{aligned}
\text { Condition (44) is }|x|^{-\delta_{I N}} \gg C & \Longleftrightarrow \delta_{I N}>0 . \\
\text { Condition (39) is } C \gg|x|^{-|\Re \sigma|+1-\delta_{O U T}} & \Longleftrightarrow|\Re \sigma|<1-\delta_{\text {OUT }} .
\end{aligned}
$$

The last condition implies that $|\Re \sigma|<1$. We also conclude that $0<\delta_{I N} \leq \delta_{\text {OUT }}<1$.

\subsection{Leading term for $\sigma= \pm\left(\theta_{0}+\theta_{x}\right), \pm\left(\theta_{0}-\theta_{x}\right) \neq 0$. Proof of (28) and (29)}

Formula (51) holds for any $\sigma \neq 0$ such that $|\Re \sigma|<1$. However, we cannot naively substitute the value of $\sigma= \pm\left(\theta_{0}+\theta_{x}\right), \pm\left(\theta_{0}-\theta_{x}\right)$, for which the coefficient of $x^{1-\sigma}$ vanishes. This is because only the leading term is certainly correct, and it may be the term in $x^{1-\sigma}$. Therefore, here we briefly give the explicit derivation of (28) and (29), using cases (99)-(102) for system (49).

Case (99), $a=0$ : This is the case $\sigma=\theta_{0}+\theta_{x} \neq 0$. The matching procedure does not change. From (99) we compute:

$$
\hat{A_{0}}=G_{0}\left(\begin{array}{cc}
\frac{\theta_{0}}{2} & r x^{\sigma} \\
0 & -\frac{\theta_{0}}{2}
\end{array}\right) G_{0}^{-1}, \quad \hat{A_{x}}=G_{0}\left(\begin{array}{cc}
\frac{\theta_{x}}{2} & -r x^{\sigma} \\
0 & -\frac{\theta_{x}}{2}
\end{array}\right) G_{0}^{-1} .
$$

This implies that $\left(\hat{A}_{0}\right)_{12}=r_{1}\left(\frac{\theta_{0}}{\theta_{0}+\theta_{x}}-\frac{r}{\theta_{0}+\theta_{x}} x^{\sigma}\right)$, while $\left(\hat{A_{1}}\right)_{12}=-r_{1}$ as in the generic case. Therefore,

$$
y(x) \sim \frac{\theta_{0}}{\theta_{0}+\theta_{x}} x-\frac{r}{\theta_{0}+\theta_{x}} x^{\sigma+1} .
$$

It is interesting to note that for $\Re \sigma>0$ we have $\hat{y}(x) \sim \theta_{0} /\left(\theta_{0}+\theta_{x}\right) x$. Such a behavior is what one would naively expect from the generic behavior (51) when $\sigma=0$.

Case (100), $b=0$, is $\sigma=-\theta_{0}-\theta_{x} \neq 0$. Case (101), $a=c$, is $\sigma=\theta_{x}-\theta_{0}$. Case (102), $b=c$, is $\sigma=\theta_{0}-\theta_{x}$. Proceeding as above, we find (28) and (29).

Remark: If we substitute $y=b_{1} x+b_{2} x^{2}+b_{3} x^{3}+\ldots$ into (PVI) we find all the coefficients $b_{n}$ by identifying equal powers of $x$. The result is (23). We need to assume that $\theta_{0} \pm \theta_{x}$ is not integer or zero. 


\subsection{Matching for $\sigma=0$. Proof of (30)}

\subsection{Case $\theta_{0} \pm \theta_{x} \neq 0$}

Lemma 5 Let $r_{1} \in \mathbf{C}, r_{1} \neq 0$. The matrices of system (40) are:

$$
\hat{A_{1}}=\left(\begin{array}{cc}
-\frac{\theta_{\infty}^{2}+\theta_{1}^{2}}{4 \theta_{\infty}} & -r_{1} \\
\frac{\left[\theta_{1}^{2}-\theta_{\infty}^{2}\right]^{2}}{16 \theta_{\infty}^{2} r_{1}} & \frac{\theta_{\infty}^{2}+\theta_{1}^{2}}{4 \theta_{\infty}}
\end{array}\right), \quad \hat{A_{0}}+\hat{A_{x}}=\left(\begin{array}{cc}
\frac{\theta_{1}^{2}-\theta_{\infty}^{2}}{4 \theta_{\infty}} & r_{1} \\
-\frac{\left[\theta_{\infty}^{2}-\theta_{1}^{2}\right]^{2}}{16 \theta_{\infty}^{2} r_{1}} & \frac{\theta_{\infty}^{2}-\theta_{1}^{2}}{4 \theta_{\infty}}
\end{array}\right), \quad \forall r_{1} \neq 0 .
$$

A fundamental matrix solution can be chosen with the following behavior at $\lambda=0$ :

$$
\Psi_{\text {OUT }}(\lambda)=\left[G_{0}+O(\lambda)\right]\left(\begin{array}{cc}
1 & \log \lambda \\
0 & 1
\end{array}\right), \quad G_{0}=\left(\begin{array}{cc}
1 & 0 \\
\frac{\theta_{\infty}^{2}-\theta_{1}^{2}}{4 \theta_{\infty} r_{1}} & \frac{1}{r_{1}}
\end{array}\right) .
$$

Proof: The system (43) is:

$$
\frac{d \Phi_{1}}{d \lambda}=\left[\frac{\hat{A}_{0}+\hat{A}_{x}}{\lambda}+\frac{\hat{A}_{1}-\frac{\theta_{1}}{2}}{\lambda-1}\right] \Phi_{1}
$$

We identify $\hat{A}_{0}+\hat{A}_{x}$ and $\hat{A}_{1}-\frac{\theta_{1}}{2}$ with $B_{0}$ and $B_{1}$ of proposition 2 in Appendix 1, diagonalizable case (98)-(102) (we recall that (103)-(107) never occur when $\theta_{\infty} \neq 0$ ) with $a=\frac{\theta_{\infty}}{2}+\frac{\theta_{1}}{2}, b=-\frac{\theta_{\infty}}{2}+\frac{\theta_{1}}{2}$, $c=0$.

The behavior of a fundamental solution is a standard result in the theory of Fuchsian systems. The matrix $G_{0}$ is defined by $G_{0}^{-1}\left(\hat{A_{0}}+\hat{A_{x}}\right) G_{0}=\left(\begin{array}{ll}0 & 1 \\ 0 & 0\end{array}\right)$.

Lemma 6 Let $r \in \mathbf{C}$. The matrices of system (46) are:

$$
\hat{\hat{A}_{0}}=\left(\begin{array}{cc}
r+\frac{\theta_{0}}{2} & \frac{4 r\left(r+\theta_{0}\right)}{\theta_{x}^{2}-\theta_{0}^{2}} \\
\frac{\theta_{0}^{2}-\theta_{x}^{2}}{4} & -r-\frac{\theta_{0}}{2}
\end{array}\right), \quad \hat{\hat{A}_{x}}=\left(\begin{array}{cc}
-r-\frac{\theta_{0}}{2} & 1-\frac{4 r\left(r+\theta_{0}\right)}{\theta_{x}^{2}-\theta_{0}^{2}} \\
\frac{\theta_{x}^{2}-\theta_{0}^{2}}{4} & r+\frac{\theta_{0}}{2} .
\end{array}\right) .
$$

There exist a fundamental solution of (46) with the following behavior at $\mu=\infty$ :

$$
\Psi_{0}(\mu)=\left[I+O\left(\frac{1}{\mu}\right)\right]\left(\begin{array}{cc}
1 & \log \mu \\
0 & 1
\end{array}\right), \quad \mu \rightarrow \infty .
$$

Proof: To compute $\hat{\hat{A}_{0}}$ and $\hat{\hat{A}_{x}}$ for the generic case, we consider the case (105) in Proposition 2, applied to the system(49). The parameters are $a=\frac{\theta_{0}}{2}+\frac{\theta_{x}}{2}, c=\theta_{0}$. In particular,

$$
\hat{\hat{A}_{0}}-\frac{\theta_{0}}{2}+\hat{\hat{A}_{x}}-\frac{\theta_{x}}{2}=\left(\begin{array}{cc}
-\frac{\theta_{0}+\theta_{x}}{2} & 1 \\
0 & -\frac{\theta_{0}+\theta_{x}}{2}
\end{array}\right)
$$

Here the values of the parameters satisfy the conditions $a \neq 0$ and $a \neq c$, namely $\theta_{0} \pm \theta_{x} \neq 0$. From the matrices (105), we obtain $\hat{\hat{A}_{0}}=B_{0}+\theta_{0} / 2$ and $\hat{\hat{A}_{x}}=B_{1}+\theta_{x} / 2$. Keeping into account (53), by the standard theory of fuchsian systems we have:

$$
\Phi_{0}(\mu)=\left[I+O\left(\frac{1}{\mu}\right)\right] \mu^{-\frac{\theta_{0}+\theta_{x}}{2}}\left(\begin{array}{cc}
1 & \log \mu \\
0 & 1
\end{array}\right), \quad \mu \rightarrow \infty .
$$

This proves the behavior of $\Psi_{0}(\mu)$.

The matching condition $\Psi_{\text {OUT }}(\lambda) \sim K_{0}(x) \Psi_{0}(\lambda / x)$ becomes:

$$
K_{0}(x)\left(\begin{array}{cc}
1 & \log \left(\frac{\lambda}{x}\right) \\
0 & 1
\end{array}\right) \sim G_{0}\left(\begin{array}{cc}
1 & \log \lambda \\
0 & 1
\end{array}\right) \Longrightarrow K_{0}(x) \sim\left(\begin{array}{cc}
\theta_{\infty}^{2}-\theta_{1}^{2} & 0 \\
\frac{4 \theta_{\infty} r_{1}}{r_{1}} & \frac{1}{r_{1}}
\end{array}\right)\left(\begin{array}{cc}
1 & \log x \\
0 & 1
\end{array}\right) .
$$

From the above result, together with (52), we compute $\hat{A}_{0}=K_{0} \hat{\hat{A}}_{0} K_{0}{ }^{-1}, \hat{A}_{1}=K_{0} \hat{\hat{A}}_{1} K_{0}{ }^{-1}$. For example,

$$
\hat{A_{0}}=G_{0}\left(\begin{array}{cc}
r+\frac{\theta_{0}}{2}+\frac{\theta_{0}^{2}-\theta_{x}^{2}}{4} \log x & \frac{\theta_{x}^{2}-\theta_{0}^{2}}{4} \log ^{2} x-2\left(r+\frac{\theta_{0}}{2}\right) \log x+\frac{4 r\left(r+\theta_{0}\right)}{\theta_{x}^{2}-\theta_{0}^{2}} \\
\frac{\theta_{0}^{2}-\theta_{x}^{2}}{4} & \frac{\theta_{x}^{2}-\theta_{0}^{2}}{4} \log x-\left(r+\frac{\theta_{0}}{2}\right)
\end{array}\right) G_{0}^{-1} .
$$


A similar expression holds for $\hat{A_{x}}$. The reader can verify that the matching conditions (39), (44) are satisfied.

The leading terms of $y(x)$ are obtained from (50) with matrix entries $\left(\hat{A}_{1}\right)_{12}=-r_{1}$ and:

$$
\left(\hat{A}_{0}\right)_{12}=r_{1}\left[\frac{\theta_{x}^{2}-\theta_{0}^{2}}{4} \log ^{2} x-2\left(r+\frac{\theta_{0}}{2}\right) \log x+\frac{4 r\left(r+\theta_{0}\right)}{\theta_{x}^{2}-\theta_{0}^{2}}\right] .
$$

The result is:

$$
y(x) \sim x\left[\frac{\theta_{x}^{2}-\theta_{0}^{2}}{4} \log ^{2} x-2\left(r+\frac{\theta_{0}}{2}\right) \log x+\frac{4 r\left(r+\theta_{0}\right)}{\theta_{x}^{2}-\theta_{0}^{2}}\right] .
$$

\subsection{Case $\theta_{0} \pm \theta_{x}=0$}

We consider here the cases (106), (107) of Proposition 2 applied to the system (49).

Case (106) is the case $\sigma=0, \theta_{0}=-\theta_{x}$, with $a=0, c=\theta_{0}$ in the system (49). From Proposition 2 we immediately have:

$$
\hat{\hat{A}_{0}}=\left(\begin{array}{cc}
\frac{\theta_{0}}{2} & r \\
0 & -\frac{\theta_{0}}{2}
\end{array}\right), \quad \hat{\hat{A}_{x}}=\left(\begin{array}{cc}
\frac{\theta_{x}}{2} & 1-r \\
0 & -\frac{\theta_{x}}{2}
\end{array}\right) .
$$

The behavior of $\Psi_{0}$ and $\Psi_{O U T}$, and the matching are the same of subsection 4.5. We obtain the same $K_{0}(x)$. Therefore:

$$
\left(\hat{A_{0}}\right)_{12}=r_{1}\left(r-\theta_{0} \ln x\right), \quad\left(\hat{A_{1}}\right)_{12}=-r_{1} .
$$

This gives the leading terms:

$$
y(x) \sim x\left(r-\theta_{0} \ln x\right)=x\left(r+\theta_{x} \ln x\right) .
$$

In the same way, we treat the other cases. Case (106) with $a=c$, is the case $\sigma=0, \theta_{0}=\theta_{x}$. As above, we find $y(x) \sim x\left(r-\theta_{0} \ln x\right)=x\left(r-\theta_{x} \ln x\right)$. Case (107) with $a=0$, is the case $\sigma=0$, $\theta_{0}=-\theta_{x}$. We find $y(x) \sim x\left(r+\theta_{0} \ln x\right)=x\left(r-\theta_{x} \ln x\right)$. Case (107) with $a=c$, is the case $\sigma=0$, $\theta_{0}=\theta_{x}$. We find $y(x) \sim x\left(r+\theta_{0} \ln x\right)=x\left(r+\theta_{x} \ln x\right)$.

Both (54) and (55) contain more than one term, and in principle only the leading one is certainly correct. To prove that they are all correct, we observe that (54) and (55) can be obtained also by direct substitution of $y(x)=x\left(A_{1}+B_{1} \ln x+C_{1} \ln ^{2} x+D_{1} \ln ^{3} x+\ldots\right)+x^{2}\left(A_{2}+B_{2} \ln x+\ldots\right)+\ldots$ into (PVI). We can recursively determine the coefficients by identifying the same powers of $x$ and $\ln x$. As a result we obtain only the five cases (31), which include (54) and (55).

\subsection{No Naive Matching for $\sigma=1$}

The condition $|\Re \sigma|<1$ suggests that the matching above does not work in the case $\sigma=1$ (and $\sigma=-1$, being equivalent). Let us convince ourselves of this fact by repeating the procedure above. A fundamental matrix solution for (40) at $\lambda=0$ is non-generic:

$$
\Psi_{\text {OUT }}(\lambda)=\left(G_{0}+O(\lambda)\right)\left(\begin{array}{cc}
\lambda^{\frac{1}{2}} & 0 \\
0 & \lambda^{-\frac{1}{2}}
\end{array}\right)\left(\begin{array}{cc}
1 & \log \lambda \\
0 & 1
\end{array}\right) .
$$

where:

$$
G_{0}=\left(\begin{array}{cc}
1 & \frac{4}{\theta_{1}^{2}-\left(\theta_{\infty}-1\right)^{2}} \\
\frac{\left(\theta_{\infty}+1\right)^{2}-\theta_{1}^{2}}{4 \theta_{\infty} r_{1}} & -\frac{1}{\theta_{\infty} r_{1}}
\end{array}\right), \quad \forall r_{1} \neq 0 .
$$

A fundamental matrix solution of (46) at $\mu=\infty$ is non-generic:

$$
\Psi_{0}(\mu)=\left(I+O\left(\frac{1}{\mu}\right)\right)\left(\begin{array}{cc}
\mu^{\frac{1}{2}} & 0 \\
0 & \mu^{-\frac{1}{2}}
\end{array}\right)\left(\begin{array}{cc}
1 & 0 \\
R \log \mu & 1
\end{array}\right)
$$

where:

$$
R:=\left(\hat{\hat{A}}_{x}\right)_{21}=\frac{\left[\left(\theta_{0}+\theta_{x}\right)^{2}-1\right]\left[\left(\theta_{0}-\theta_{x}\right)^{2}-1\right]}{16 r}, \quad r \neq 0 .
$$


The matching relation:

$$
K_{0}(x)\left(\begin{array}{cc}
\left(\frac{\lambda}{x}\right)^{\frac{1}{2}} & 0 \\
R\left(\frac{\lambda}{x}\right)^{-\frac{1}{2}} \log \left(\frac{\lambda}{x}\right) & \left(\frac{\lambda}{x}\right)^{-\frac{1}{2}}
\end{array}\right) \sim\left(\begin{array}{cc}
1 & \frac{4}{\theta_{1}^{2}-\left(\theta_{\infty}-1\right)^{2}} \\
\frac{\left(\theta_{\infty}+1\right)^{2}-\theta_{1}^{2}}{4 \theta_{\infty} r_{1}} & -\frac{1}{\theta_{\infty} r_{1}}
\end{array}\right)\left(\begin{array}{cc}
\lambda^{\frac{1}{2}} & \lambda^{\frac{1}{2}} \log \lambda \\
0 & \lambda^{-\frac{1}{2}}
\end{array}\right),
$$

shows that we cannot eliminate $\lambda$ to obtain $K_{0}(x)$.

One case $\sigma=1$ is studied in Part III, making use of a non-fuchsian reductions of the system (4).

\subsection{Monodromy Data}

Systems (40) (46) are equivalent to Gauss hyper-geometric equations, as it is explained in Appendix 1 (make use of the systems (43) and (49) respectively). Therefore, the monodromy can be computed in a standard way, using the connection formulae for the hyper-geometric functions.

We obtain in this way the monodromy of $\Psi_{O U T}$ and $\Psi_{I N}$. As it is explained in section 2.2 , it may be necessary to do a transformation $\Psi_{O U T} \mapsto \Psi_{\text {OUT }}^{M a t c h}:=\Psi_{\text {OUT }} C_{O U T}$, in order to match the "out" and "in" solutions with a solution $\Psi$ of (4). In this way, the monodromy matrices $M_{0}, M_{x}$, $M_{1}$ of $\Psi$ can be obtained. They depend on $r$. We then compute the traces of $M_{i} M_{j}$ and extract $r$, which is thus obtained as a function of the monodromy data.

We do not repeat the computations here. One example is the computation of (37) in [17] and [8] [9] [11] [5].

\section{PART III - Derivations for Non-fuchsian Reduction}

\section{$5 \quad$ Case $\theta_{1}= \pm \theta_{\infty}, \theta_{x}= \pm \theta_{0}, \lim _{x \rightarrow 0}\left(A_{x}+A_{0}\right)=0$. Solution (19)}

We begin by observing that for $\theta_{x}= \pm \theta_{0}$, system (49) may fall in cases (103) and (104). If it is so, then $\hat{\hat{A}_{0}}+\hat{\hat{A_{x}}}=0$, and therefore $\hat{A_{0}}+\hat{A_{x}}=0$. More precisely, we start from the following hypotheses:

$$
\begin{gathered}
\lim _{x \rightarrow 0}\left(A_{0}(x)+A_{x}(x)\right)=0 \\
A:=\lim _{x \rightarrow 0} A_{x}(x)=\text { a constant matrix with eigenvalues } \pm \frac{\theta_{x}}{2}
\end{gathered}
$$

The first hypothesis means that we can write (the trace is zero):

$$
A_{0}+A_{x}=\left(\begin{array}{cc}
a(x) & b(x) r \\
c(x) \frac{1}{r} & -a(x)
\end{array}\right), \quad \lim _{x \rightarrow 0} a(x)=\lim _{x \rightarrow 0} b(x)=\lim _{x \rightarrow 0} c(x)=0,
$$

The second hypotheses implies that the general form of $A$ is:

$$
A=\left(\begin{array}{cc}
s+\frac{\theta_{x}}{2} & -r \\
\frac{\left(s+\theta_{x}\right) s}{r} & -s-\frac{\theta_{x}}{2}
\end{array}\right), \quad r, s \in \mathbf{C}, \quad r \neq 0 .
$$

We also write:

$$
A_{x}(x)-A=: \Delta_{x}(x), \quad A_{0}+A=: \Delta_{0}(x), \quad \Delta_{0}+\Delta_{x} \equiv A_{0}+A_{x} .
$$

$\Delta_{x}(x)$ and $\Delta_{0}(x)$ are vanishing. We suppose that the slowest vanishing behavior be of order $x^{\sigma_{0}}$, for some $\sigma_{0}>0$. Namely:

$$
a(x), b(x), c(x),\left(\Delta_{x}\right)_{i j}(x),\left(\Delta_{0}\right)_{i j}(x)=O\left(x^{\sigma_{0}}\right), \quad \sigma_{0}>0 .
$$

Finally, we have:

$$
A_{1}(x)=-\frac{\theta_{\infty}}{2} \sigma_{3}-\left(A_{0}+A_{x}\right) \longrightarrow-\frac{\theta_{\infty}}{2} \sigma_{3}, \quad x \rightarrow 0 .
$$




\subsection{Coalescence of Singularities}

\section{1) THE SYSTEM for $\Psi_{O U T}$.}

We consider system (11), in the domain $|\lambda| \geq|x|^{\delta_{\text {OUT }}}$. Let us determine the conditions to neglect a term $x^{n} A_{x} / \lambda^{n+1}$ - and all the terms following it - with respect to $\left(A_{0}+A_{x}\right) / \lambda$, when $x \rightarrow 0$, $\lambda \sim x^{\delta}, \delta \leq \delta_{\text {OUT }}$.

$$
\text { We can neglect } \frac{x^{n} A_{x}}{\lambda^{n+1}} \Longleftrightarrow\left|\frac{x^{n}}{\lambda^{n}} A_{x}\right| \ll\left|\left(A_{0}+A_{x}\right)_{i j}\right|, \quad \forall i, j \in\{1,2\} \text {. }
$$

Since $\lim _{x \rightarrow 0}\left(A_{x}\right)_{i j}$ are non-zero constants, the above condition is: $|x|^{n-n \delta} \ll|x|^{\sigma_{0}}$, namely: $\delta<$ $1-\sigma_{0} / n$. We state this result as a lemma.

Lemma 7 Let $N_{O U T} \geq 2$ be an integer. We can approximate (11) with:

$$
\frac{d \Psi_{\text {OUT }}}{d \lambda}=\left[\frac{\left(A_{0}+A_{x}\right)}{\lambda}+\frac{A_{x}}{\lambda} \sum_{n=1}^{N_{\text {OUT }}-1}\left(\frac{x}{\lambda}\right)^{n}+\frac{A_{1}}{\lambda-1}\right] \Psi_{\text {OUT }} .
$$

if and only if:

$$
\delta_{\text {OUT }}<1-\frac{\sigma_{0}}{N_{\text {OUT }}} .
$$

Suppose that all term $x^{n} A_{x} / \lambda^{n+1}$, with $n \geq N_{O U T}$, have been neglected. We can also make the substitution $A_{1} \mapsto-\frac{\theta_{\infty}}{2} \sigma_{3}$ in $\frac{A_{1}}{\lambda-1}$, if and only if the error term $-\frac{A_{0}+A_{x}}{\lambda-1}$, is smaller than $\frac{x^{N O U T^{-1}} A_{x}}{\lambda^{N_{O U T}}}$. Namely, if and only if:

$$
\left|\left(A_{0}+A_{x}\right)_{i j}\right| \ll\left|\frac{x^{N_{\text {OUT }}-1}\left(A_{x}\right)_{i j}}{\lambda^{N_{\text {OUT }}}}\right| .
$$

This is $|x|^{\sigma_{0}} \ll|x|^{N_{\text {OUT }}-1-N_{\text {OUT }} \delta_{\text {OUT }}}$, namely $\delta_{\text {OUT }}>1-\frac{1+\sigma_{0}}{N_{\text {OUT }}}$.

We can also do the substitution $A_{x} \mapsto A$, provided that $\delta_{\text {OUT }}<1$. This is because we can neglecting terms $\frac{x^{n} \Delta_{x}}{\lambda^{n+1}}$ with respect to $\frac{A_{0}+A_{x}}{\lambda}$, where both $\Delta_{x}$ and $A_{0}+A_{x}$ are $O\left(x^{\sigma_{0}}\right), \lambda \sim x^{\delta}$, $\delta<1$. We summarize the result in the following lemma.

Lemma 8 Let $N_{O U T} \geq 2$ be integer. We can approximate (11) with:

$$
\frac{d \Psi_{\text {OUT }}}{d \lambda}=\left[\frac{A_{0}+A_{x}}{\lambda}+\frac{A}{\lambda} \sum_{n=1}^{N_{O U T}-1}\left(\frac{x}{\lambda}\right)^{n}-\frac{\theta_{\infty}}{2} \frac{\sigma_{3}}{\lambda-1}\right] \Psi_{O U T} .
$$

if and only if:

$$
1-\frac{1+\sigma_{0}}{N_{O U T}}<\delta_{\text {OUT }}<1-\frac{\sigma_{0}}{N_{O U T}}
$$

In particular, this means that $\delta_{\text {OUT }}<1$.

\section{Example:}

If $\sigma_{0}=1$ and $N_{I N}=2$ we have:

$$
\frac{d \Psi_{\text {OUT }}}{d \lambda}=\left[\frac{x A}{\lambda^{2}}+\frac{A_{0}+A_{x}}{\lambda}-\frac{\theta_{\infty}}{2} \frac{\sigma_{3}}{\lambda-1}\right] \Psi_{\text {OUT }}, \quad 0<\delta_{\text {OUT }}<\frac{1}{2} .
$$

If $\sigma_{0}=1$ and $N_{I N}=3$ we have:

$$
\frac{d \Psi_{\text {OUT }}}{d \lambda}=\left[\frac{x^{2} A}{\lambda^{3}}+\frac{x A}{\lambda^{2}}+\frac{A_{0}+A_{x}}{\lambda}-\frac{\theta_{\infty}}{2} \frac{\sigma_{3}}{\lambda-1}\right] \Psi_{\text {OUT }}, \quad \frac{1}{3}<\delta_{\text {OUT }}<\frac{2}{3} .
$$

\section{2) THE SYSTEM for $\Psi_{I N}$.}


We consider system (12) in the domain $|\lambda| \leq|x|^{\delta_{I N}}$. We investigate the condition necessary and sufficient to neglect a term $\lambda^{n} A_{1}$ (and all its next terms) with respect to $\frac{A_{0}}{\lambda}+\frac{A_{x}}{\lambda-x}$. It is convenient to write:

$$
\frac{A_{0}}{\lambda}+\frac{A_{x}}{\lambda-x}=\frac{A_{0}+A_{x}}{\lambda-x}-\frac{x A_{0}}{\lambda(\lambda-x)} .
$$

Suppose that $\lambda \sim x^{\delta}, \delta \geq \delta_{I N}$.

We neglect $\quad A_{1} \lambda^{n} \Longleftrightarrow\left\{\begin{array}{l}\left|\frac{x A_{0}}{\lambda(\lambda-x)}\right| \gg\left|A_{1} \lambda^{n}\right|, \text { namely: }|x|^{1-2 \delta} \gg|x|^{n \delta} \Leftrightarrow \delta>\frac{1}{n+2} ; \\ \left|\frac{A_{0}+A_{x}}{\lambda-x}\right| \gg\left|A_{1} \lambda^{n}\right|, \text { namely: }|x|^{\sigma_{0}-\delta} \gg|x|^{n \delta} \Leftrightarrow \delta>\frac{\sigma_{0}}{n+1} .\end{array}\right.$

Thus, we have the condition $\delta>\max \left\{\frac{\sigma_{0}}{n+1}, \frac{1}{n+2}\right\}$. We have proven the following:

Lemma 9 Let $N_{I N} \geq 1$ be an integer. We approximate (12) with:

$$
\frac{d \Psi_{I N}}{d \lambda}=\left[\frac{A_{0}}{\lambda}+\frac{A_{x}}{\lambda-x}-A_{1} \sum_{n=0}^{N_{I N}-1} \lambda^{n}\right] \Psi_{I N} .
$$

if and only if:

$$
\delta_{I N}>\max \left\{\frac{\sigma_{0}}{N_{I N}+1}, \frac{1}{N_{I N}+2}\right\} .
$$

We further make the substitution $A_{1} \mapsto-\frac{\theta_{\infty}}{2} \sigma_{3}$. This is possible if and only if two conditions are true: 1) $\left|\frac{x A_{0}}{\lambda(\lambda-x)}\right|$ and $\left|\frac{A_{0}+A_{x}}{\lambda-x}\right|$ are dominant w.r.t. the term $A_{0}+A_{x}$ appearing in $A_{1}=$ $-\frac{\theta_{\infty}}{2} \sigma_{3}-\left(A_{0}+A_{x}\right)$. 2) $\left|\lambda^{N_{I N}-1} A_{1}\right|$, i.e. $\left|\lambda^{N_{I N}-1} \sigma_{3}\right|$, is dominant w.r.t. the term $A_{0}+A_{x}$ in $A_{1}$. Esplicitely, the conditions are:

$$
\begin{gathered}
\left|\frac{A_{0}+A_{x}}{\lambda-x}\right| \gg\left|A_{0}+A_{x}\right| \text { (this is always true), } \\
\left|\frac{x A_{0}}{\lambda(\lambda-x)}\right| \gg\left|A_{0}+A_{x}\right| \Longleftrightarrow|x|^{1-2 \delta}>x^{\sigma_{0}} \text {, namely: } \delta>\frac{1-\sigma_{0}}{2},
\end{gathered}
$$

and

$$
\left|\lambda^{N_{I N}-1} \sigma_{3}\right| \gg\left|A_{0}+A_{x}\right| \Longleftrightarrow|x|^{\left(N_{I N}-1\right) \delta}>|x|^{\sigma_{0}} \text {, namely: } \delta<\frac{\sigma_{0}}{N_{I N}-1} .
$$

We have:

Lemma 10 Let $N_{I N} \geq 1$ be an integer. We approximate (12) with:

$$
\begin{aligned}
& \frac{d \Psi_{I N}}{d \lambda}=\left[\frac{A_{0}}{\lambda}+\frac{A_{x}}{\lambda-x}+\frac{\theta_{\infty}}{2} \sigma_{3} \sum_{n=0}^{N_{I N}-1} \lambda^{n}\right] \Psi_{I N} \\
= & {\left[\frac{A_{0}+A_{x}}{\lambda-x}-\frac{x A_{0}}{\lambda(\lambda-x)}+\frac{\theta_{\infty}}{2} \sigma_{3} \sum_{n=0}^{N_{I N}-1} \lambda^{n}\right] \Psi_{I N}, }
\end{aligned}
$$

if and only if:

$$
\max \left\{\frac{1-\sigma_{0}}{2}, \frac{\sigma_{0}}{N_{I N}+1}, \frac{1}{N_{I N}+2}\right\}<\delta_{I N}<\frac{\sigma_{0}}{N_{I N}-1} .
$$

As a final simplification, we substitute $A_{0}=-A+\Delta_{0} \mapsto-A$. This is possible if and only if:

$$
\left|\frac{x \Delta_{0}}{\lambda(\lambda-x)}\right| \ll\left|\frac{A_{0}+A_{x}}{\lambda-x}\right| \Longleftrightarrow|x|^{1+\sigma_{0}-2 \delta}<|x|^{\sigma_{0}-\delta} \text {, namely } \delta<1,
$$

and:

$$
\left|\frac{x \Delta_{0}}{\lambda(\lambda-x)}\right| \ll\left|\lambda^{N_{I N}-1} \frac{\theta_{\infty}}{2} \sigma_{3}\right| \Longleftrightarrow|x|^{1+\sigma_{0}-2 \delta} \ll x^{\left(N_{I N}-1\right) \delta} \text {, namely: } \delta<\frac{\sigma_{0}+1}{N_{I N}+1} .
$$

We have proven the following: 
Lemma 11 Let $N_{I N} \geq 1$ be an integer. We can approximate (12) with:

$$
\frac{d \Psi_{I N}}{d \lambda}=\left[\frac{A_{0}+A_{x}}{\lambda-x}+\frac{x A}{\lambda(\lambda-x)}+\frac{\theta_{\infty}}{2} \sigma_{3} \sum_{n=0}^{N_{I N}-1} \lambda^{n}\right] \Psi_{I N},
$$

if and only if:

$$
\max \left\{\frac{1-\sigma_{0}}{2}, \frac{\sigma_{0}}{N_{I N}+1}, \frac{1}{N_{I N}+2}\right\}<\delta_{I N}<\min \left\{\frac{\sigma_{0}}{N_{I N}-1}, \frac{\sigma_{0}+1}{N_{I N}+1}\right\} .
$$

\section{Examples:}

If $\sigma_{0}=1$ and $N_{I N}=1$, we have:

$$
\frac{d \Psi_{I N}}{d \lambda}=\left[\frac{A_{0}+A_{x}}{\lambda-x}+\frac{x A}{\lambda(\lambda-x)}+\frac{\theta_{\infty}}{2} \sigma_{3}\right] \Psi_{I N}, \quad \frac{1}{2}<\delta_{I N}<1 .
$$

If we keep $-A_{0}$ instead of $A$, with no change in the condition on $\delta_{I N}$, we can also rewrite:

$$
\frac{d \Psi_{I N}}{d \lambda}=\left[\frac{A_{0}}{\lambda}+\frac{A_{x}}{\lambda-x}+\frac{\theta_{\infty}}{2} \sigma_{3}\right] \Psi_{I N},
$$

If $\sigma_{0}=1$ and $N_{I N}=2$, we have:

$$
\frac{d \Psi_{I N}}{d \lambda}=\left[\frac{A_{0}+A_{x}}{\lambda-x}+\frac{x A}{\lambda(\lambda-x)}+\frac{\theta_{\infty}}{2} \sigma_{3}(1+\lambda)\right] \Psi_{I N}, \quad \frac{1}{3}<\delta_{I N}<\frac{2}{3} .
$$

Equivalently, we can write:

$$
\frac{d \Psi_{I N}}{d \lambda}=\left[\frac{A_{0}}{\lambda}+\frac{A_{x}}{\lambda-x}+\frac{\theta_{\infty}}{2} \sigma_{3}(1+\lambda)\right] \Psi_{I N}
$$

\subsection{Matching}

We do the matching in the overlapping region $|x|^{\delta_{\text {OUT }}} \leq|\lambda| \leq|x|^{\delta_{I N}}$. This imposes: $\delta_{I N} \leq \delta_{\text {OUT }}$. In order for the overlapping region not to be empty, we must choose suitable reductions of (11) and (12). If we expect $\sigma_{0}$ to be close to 1 , we try to match solutions $\Psi_{O U T}$ and $\Psi_{I N}$ satisfying one of the following sets of systems:

First choice:

$$
\begin{aligned}
\frac{d \Psi_{O U T}}{d \lambda} & =\left[\frac{x A}{\lambda^{2}}+\frac{A_{0}+A_{x}}{\lambda}-\frac{\theta_{\infty}}{2} \frac{\sigma_{3}}{\lambda-1}\right] \Psi_{O U T}, \\
\frac{d \Psi_{I N}}{d \lambda} & =\left[\frac{A_{0}}{\lambda}+\frac{A_{x}}{\lambda-x}+\frac{\theta_{\infty}}{2} \sigma_{3}(1+\lambda)\right] \Psi_{I N} .
\end{aligned}
$$

The condition to be satisfied for $\sigma_{0} \cong 1$ is: $\frac{\sigma_{0}}{3}<\delta_{I N} \leq \delta_{O U T}<1-\frac{\sigma_{0}}{2}$. For $\sigma_{0}=1$, this is:

$$
\frac{1}{3}<\delta_{I N} \leq \delta_{\text {OUT }}<\frac{1}{2}
$$

Second choice:

$$
\begin{gathered}
\frac{d \Psi_{\text {OUT }}}{d \lambda}=\left[\frac{x^{2} A}{\lambda^{3}}+\frac{x A}{\lambda^{2}}+\frac{A_{0}+A_{x}}{\lambda}-\frac{\theta_{\infty}}{2} \frac{\sigma_{3}}{\lambda-1}\right] \Psi_{\text {OUT }}, \\
\frac{d \Psi_{I N}}{d \lambda}=\left[\frac{A_{0}}{\lambda}+\frac{A_{x}}{\lambda-x}+\frac{\theta_{\infty}}{2} \sigma_{3}\right] \Psi_{I N} .
\end{gathered}
$$

For $\sigma_{0} \cong 1$, the condition to be satisfied is: $\frac{\sigma_{0}}{2}<\delta_{I N} \leq \delta_{O U T}<1-\frac{\sigma_{0}}{3}$. For $\sigma_{0}=1$, this is:

$$
\frac{1}{2}<\delta_{I N} \leq \delta_{\text {OUT }}<\frac{2}{3} .
$$

In both cases, the overlapping regions are not empty. The matching procedure will determine the leading terms (order $x^{\sigma_{0}}$ ) of the unknown matrix elements $a(x), b(x), c(x)$ of $A_{0}+A_{x}$. 


\subsection{Matching for the First Choice: $\frac{1}{3}<\delta_{I N} \leq \delta_{O U T}<\frac{1}{2}$.}

We rewrite the systems in a more convenient form:

$$
\begin{gathered}
\nu:=\frac{1}{\lambda}, \quad \mu:=\frac{\lambda}{x} ; \\
\frac{d \Psi_{\text {OUT }}}{d \nu}=\left[-x A-\frac{A_{0}+A_{x}}{\nu}-\frac{\theta_{\infty}}{2} \frac{\sigma_{3}}{\nu(\nu-1)}\right] \Psi_{\text {OUT }} \\
\frac{d \Psi_{I N}}{d \mu}=\left[x^{2} \frac{\theta_{\infty}}{2} \sigma_{3} \mu+x \frac{\theta_{\infty}}{2} \sigma_{3}+\frac{A_{0}}{\mu}+\frac{A_{x}}{\mu-1}\right] \Psi_{I N} \\
=\left[x^{2} \frac{\theta_{\infty}}{2} \sigma_{3} \mu+x \frac{\theta_{\infty}}{2} \sigma_{3}+\frac{A_{0}+A_{x}}{\mu}-\frac{A_{0}}{\mu(\mu-1)}\right] \Psi_{I N} .
\end{gathered}
$$

Then we substitute $A_{0} \mapsto-A$ in the last term.

In the matching region $|x|^{-\delta_{I N}} \leq|\nu| \leq|x|^{-\delta_{\text {OUT }}},|x|^{\delta_{\text {OUT }}-1} \leq \mu \leq|x|^{\delta_{I N}-1}$, we have $\nu \rightarrow \infty$, $\mu \rightarrow \infty$. The point at infinity is a non-fuchsian singularity. ${ }^{5}$ In order to find the local behavior at this point, it is convenient to put the leading term in diagonal form. Let $G$ be the invertible matrix such that:

$$
G^{-1} A G=-\frac{\theta_{x}}{2} \sigma_{3}, \quad \text { for example: } G=\left(\begin{array}{cc}
1 & \frac{r}{s} \\
\frac{s+\theta_{x}}{r} & 1
\end{array}\right)
$$

and put:

$$
\Psi_{\text {OUT }}=: G \tilde{\Psi}_{O U T}
$$

Then:

$$
\begin{gathered}
\frac{d \tilde{\Psi}_{\text {OUT }}}{d \nu}=\left[x \frac{\theta_{x}}{2} \sigma_{3}-\frac{G^{-1}\left(A_{0}+A_{x}\right) G}{\nu}-\frac{\theta_{\infty}}{2} G^{-1} \sigma_{3} G\left(\frac{1}{\nu^{2}}+\frac{1}{\nu^{3}}+\ldots\right)\right] \tilde{\Psi}_{\text {OUT }}, \quad \nu \rightarrow \infty ; \\
\frac{d \Psi_{I N}}{d \mu}=\left[x^{2} \frac{\theta_{\infty}}{2} \sigma_{3} \mu+x \frac{\theta_{\infty}}{2} \sigma_{3}+\frac{A_{0}+A_{x}}{\mu}+A\left(\frac{1}{\mu^{2}}+\frac{1}{\mu^{3}}+\ldots\right)\right] \Psi_{I N}, \quad \mu \rightarrow \infty .
\end{gathered}
$$

In order to write the local behavior of $\tilde{\Psi}_{O U T}$ and $\Psi_{I N}$ at infinity, we observe that the systems (60) and (61) respectively have the following forms:

$$
\begin{gathered}
\frac{d Y_{1}}{d z}=\left[\Omega+\frac{D_{1}}{z}+\frac{D_{2}}{z^{2}}+\frac{D_{3}}{z^{3}}+\ldots\right] Y_{1}, \\
\frac{d Y_{2}}{d z}=\left[x^{2} \Lambda z+x \Lambda z+\frac{E_{1}}{z}+\frac{E_{2}}{z^{2}}+\frac{E_{3}}{z^{3}}+\ldots\right] Y_{2},
\end{gathered}
$$

where $\Omega$ and $\Lambda$ are diagonal matrices with distinct eigenvalues. In our case:

$$
\Omega=x \frac{\theta_{x}}{2} \sigma_{3}, \quad \Lambda=\frac{\theta_{\infty}}{2} \sigma_{3} .
$$

The eigenvalues are distinct iff $\theta_{x} \neq 0, \theta_{\infty} \neq 0$.

\footnotetext{
${ }^{5}$ System (59) can be also written with $\frac{\theta_{\infty}}{2} \sigma_{3} \mapsto-A_{1}$ :

$$
\begin{gathered}
\frac{d \Psi_{O U T}}{d \nu}=\left[-x A-\frac{A_{0}+A_{x}}{\nu}+\frac{A_{1}}{\nu(\nu-1)}\right] \Psi_{O U T} \\
{\left[-x A-\frac{A_{0}+A_{x}+A_{1}}{\nu}+\frac{A_{1}}{\nu-1}\right] \Psi_{O U T}}
\end{gathered}
$$

After diagonalization, we get:

$$
\frac{d \tilde{\Psi}_{O U T}}{d \nu}=\left[x \frac{\theta_{x}}{2} \sigma_{3}+\frac{\theta_{\infty}}{2} \frac{G^{-1} \sigma_{3} G}{\nu}+\frac{G^{-1} A_{1} G}{\nu-1}\right] \tilde{\Psi}_{O U T} .
$$
}

This form is that of a system of isomonodromy deformation for the fifth Painlevé equation. 
The theory for such systems is developed in [3] (see also [4]). For any sector of angular width $\pi+\epsilon, \epsilon>0$ sufficiently small, there exists a unique solution of (62) with asymptotic expansion:

$$
\begin{gathered}
Y_{1}(z) \sim\left[I+\frac{G_{1}}{z}+\frac{G_{2}}{z^{2}}+\ldots\right] \exp \{\Omega z\} z^{\Omega_{1}}, \quad z \rightarrow \infty . \\
\Omega_{1}=\text { diagonal part of } D_{1} .
\end{gathered}
$$

For any sector of angular width $\frac{\pi}{2}+\epsilon, \epsilon>0$ sufficiently small, there exist a unique solution of (63) with asymptotic expansion:

$$
\begin{gathered}
Y_{2}(z) \sim\left[I+\frac{K_{1}}{z}+\frac{K_{2}}{z^{2}}+\ldots\right] \exp \left\{\frac{x^{2}}{2} \Lambda z^{2}+x \Lambda z\right\} z^{\Lambda_{1}}, \quad z \rightarrow \infty . \\
\Lambda_{1}=\text { diagonal part of } E_{1} .
\end{gathered}
$$

We can always find two solutions $Y_{1}(z)$ and $Y_{2}(z)$ as above, such that the sectors where the asymptotic expansions hold are overlapping. We refer the reader to [3] for the general description of irregular system with a Stokes phenomenon, and to the Appendix 2 for the computation of the matrices $G_{i}, K_{i}, i=1,2, \ldots$

The systems (60), (61) are isomonodromic. This imposes that $\Omega_{1}$ and $\Lambda_{1}$ must be independent of $x$. They are:

$$
\begin{gathered}
\Omega_{1}=\text { diagonal of }\left(-G^{-1}\left(A_{0}+A_{x}\right) G\right), \\
\Lambda_{1}=\text { diagonal of }\left(A_{0}+A_{x}\right)=\left(\begin{array}{cc}
a(x) & 0 \\
0 & -a(x)
\end{array}\right) .
\end{gathered}
$$

We compute:

$$
G^{-1}\left(A_{0}+A_{x}\right) G=\frac{1}{\theta_{x}}\left(\begin{array}{cc}
-\left(2 s+\theta_{x}\right) a-s\left(s+\theta_{x}\right) b+c & \left(-2 a-s b+\frac{c}{s}\right) r \\
\frac{s}{r}\left(2\left(s+\theta_{x}\right) a+\left(s+\theta_{x}\right)^{2} b-c\right) & \left(2 s+\theta_{x}\right) a+s\left(s+\theta_{x}\right) b-c
\end{array}\right)
$$

Since $a, b, c$ vanish, the condition of isomonodromicity implies that:

$$
\Omega_{1}=0, \quad \Lambda_{1}=0
$$

This means that the leading terms of $a(x), b(x), c(x)$ satisfy the conditions:

$$
a(x)=0, \quad c(x)=s\left(s+\theta_{x}\right) b(x) .
$$

The above conditions mean that if $b(x), c(x)=O\left(x^{\sigma_{0}}\right)$, then $a(x)$ is of higher order, i.e. it vanishes faster than $x^{\sigma_{0}}$. Note that with this choice of $a, b, c$ we get:

$$
G^{-1}\left(A_{0}+A_{x}\right) G=\left(\begin{array}{cc}
0 & b r \\
\frac{s\left(s+\theta_{x}\right)}{r} b & 0
\end{array}\right)
$$

We are ready to write the behavior of $\Psi_{O U T}$ :

$$
\begin{gathered}
\Psi_{\text {OUT }}=G\left[I+\frac{G_{1}}{\nu}+\frac{G_{2}}{\nu^{2}}+\ldots\right] \exp \left\{x \frac{\theta_{x}}{2} \sigma_{3} \nu\right\}, \quad \nu \rightarrow \infty \\
=G\left[I+G_{1} \lambda+G_{2} \lambda^{2}+\ldots\right] \exp \left\{\frac{\theta_{x}}{2} \sigma_{3} \frac{x}{\lambda}\right\}, \quad \lambda \rightarrow 0 .
\end{gathered}
$$

We use the formulae of Appendix 2 to determine $G_{1}$ :

$$
\left(G_{1}\right)_{i j}=2 \frac{\left[G^{-1}\left(A_{0}+A_{x}\right) G\right]_{i j}}{x \theta_{x}\left(\left(\sigma_{3}\right)_{i i}-\left(\sigma_{3}\right)_{j j}\right)}, \quad i \neq j
$$




$$
\left(G_{1}\right)_{i i}=\frac{\theta_{\infty}}{2}\left(G^{-1} \sigma_{3} G\right)_{i j}+2 \frac{\left[G^{-1}\left(A_{0}+A_{x}\right) G\right]_{i j}\left[G^{-1}\left(A_{0}+A_{x}\right) G\right]_{j i}}{x \theta_{x}\left(\left(\sigma_{3}\right)_{j j}-\left(\sigma_{3}\right)_{i i}\right)} .
$$

In the second term of the last formula $j=2$ if $i=1, j=1$ if $i=2$. We compute:

$$
G^{-1} \sigma_{3} G=\frac{1}{\theta_{x}}\left(\begin{array}{cc}
-\left(2 s+\theta_{x}\right) & -2 r \\
\frac{2 s\left(s+\theta_{x}\right)}{r} & 2 s+\theta_{x}
\end{array}\right) .
$$

Therefore:

$$
\begin{gathered}
\left(G_{1}\right)_{12}=\frac{r}{x \theta_{x}} b, \quad\left(G_{1}\right)_{21}=-\frac{s\left(s+\theta_{x}\right)}{x \theta_{x} r} b, \\
\left(G_{1}\right)_{11}=-\frac{\theta_{\infty}}{2 \theta_{x}}\left(2 s+\theta_{x}\right)+\frac{s\left(s+\theta_{x}\right)}{x \theta_{x}} b^{2}, \quad\left(G_{1}\right)_{22}=-\left(G_{1}\right)_{11}
\end{gathered}
$$

On the other hand, the local behavior of $\Psi_{I N}$ is:

$$
\begin{array}{cc}
\Psi_{I N}=\left[I+\frac{K_{1}}{\mu}+\frac{K_{2}}{\mu^{2}}+\ldots\right] \exp \left\{x^{2} \frac{\theta_{\infty}}{4} \sigma_{3} \mu^{2}+x \frac{\theta_{\infty}}{2} \sigma_{3} \mu\right\}, \quad \mu \rightarrow \infty \\
=\left[I+K_{1} \frac{x}{\mu}+K_{2} \frac{x^{2}}{\lambda^{2}}+\ldots\right] \exp \left\{\frac{\theta_{\infty}}{4} \sigma_{3} \lambda^{2}+\frac{\theta_{\infty}}{2} \sigma_{3} \lambda\right\}, \quad \lambda \rightarrow 0 .
\end{array}
$$

We determine $K_{1}$ from the formulas of Appendix 2 .

$$
K_{1}=\text { diagonal part of }(-A)=\left(\begin{array}{cc}
-\left(s+\frac{\theta_{x}}{2}\right) & 0 \\
0 & s+\frac{\theta_{x}}{2}
\end{array}\right)
$$

The matching conditition:

$$
\Psi_{\text {OUT }}(\lambda, x) \sim \Psi_{I N}(\lambda, x), \quad x \rightarrow 0, \quad|x|^{\delta_{O U T}} \leq|\lambda| \leq|x|^{\delta_{I N}} .
$$

is restricted to the overlapping sector where both expansions of $\Psi_{O U T}$ and $\Psi_{I N}$ hold. Noting that $\Psi_{\text {OUT }} \sim G$ and $\Psi_{I N} \sim I$, we choose the new solution $\Psi_{\text {OUT }} \mapsto \Psi_{\text {OUT }} G^{-1}$. Then, we expand the exponents:

$$
\Psi_{O U T}=\left[I+G G_{1} G^{-1} \lambda+G G_{2} G^{-1} \lambda^{2}+\ldots\right]\left[I+\frac{\theta_{x}}{2} G \sigma_{3} G^{-1} \frac{x}{\lambda}+\frac{\theta_{x}^{2}}{8} \frac{x^{2}}{\lambda^{2}}+\ldots\right] .
$$

The point here is quite delicate. We consider the relation of dominance among terms - and write the leading terms of the expansion - as they are in case the $G_{n}(x)$ 's are not divergent when $x \rightarrow 0$. Keeping into account that $\frac{\theta_{x}}{2} G \sigma_{3} G^{-1}=-A$, the dominant terms are:

$$
\Psi_{\text {OUT }}(\lambda, x)=I+G G_{1} G^{-1} \lambda-A \frac{x}{\lambda}+O\left(\lambda^{2}, \frac{x^{2}}{\lambda^{2}}, x\right)
$$

It is important to note that $\lambda$ is dominant w.r.t $\frac{x}{\lambda}$, because $\delta_{\text {OUT }}<\frac{1}{2}$; namely, $\lambda \sim x^{\delta}$ vanishes slower than $\frac{x}{\lambda} \sim x^{1-\delta}$, as $x \rightarrow 0$.

We expand the exponent in $\Psi_{I N}$, and keep only the first dominant terms (in the spirit of the observation on the dominance relations made above):

$$
\Psi_{I N}(\lambda, x)=I+\frac{\theta_{\infty}}{2} \sigma_{3} \lambda+K_{1} \frac{x}{\lambda}+O\left(\lambda^{2}, \frac{x^{2}}{\lambda^{2}}, x\right)
$$

$\Psi_{\text {OUT }}$ and $\Psi_{I N}$ match in the first term $I$. We impose the matching of the second term, namely the term in $\lambda$ :

$$
G G_{1}(x) G^{-1} \sim \frac{\theta_{\infty}}{2} \sigma_{3}, \quad x \rightarrow 0 .
$$


Namely:

$$
G_{1}(x) \sim \frac{\theta_{\infty}}{2} G^{-1} \sigma_{3} G, \quad x \rightarrow 0
$$

From the explicit form of $G_{1}$ and $G^{-1} \sigma_{3} G$ given above, we conclude that the matching is satisfied if and only if:

$$
b(x) \sim-x \theta_{\infty}, \quad \text { and } \sigma_{0}=1 .
$$

The error in $b(x)$ is of higher order w.r.t. $x$. The determination of the leading behavior of $A_{0}+A_{x}$ is complete, because $c(x) \sim s\left(s+\theta_{x}\right) b(x), x \rightarrow 0$. Namely:

$$
c(x) \sim-x\left\{s\left(s+\theta_{x}\right) \theta_{\infty}\right\}, \quad a(x)=o(x) .
$$

With such a choice of $b(x)$, one can verify that the terms in $\Psi_{O U T}$ and $\Psi_{I N}$ which follow the second (i.e. which follow the term in $\lambda$ ) are actually of higher order in $x$. Nevertheless, $\Psi_{O U T}$ and $\Psi_{I N}$ match only in the first and second term, being already the off-diagonal entries of the third term not matching (i.e. $-A$ and $K_{1}=$ diagonal part of $-A$ respectively.)

\subsection{Matching for the Second Choice: $\frac{1}{2}<\delta_{I N} \leq \delta_{O U T}<\frac{2}{3}$.}

We rewrite the systems in the convenient form:

$$
\begin{gathered}
\nu:=\frac{1}{\lambda}, \quad \mu:=\frac{\lambda}{x} \\
\frac{d \Psi_{\text {OUT }}}{d \nu}=\left[-x^{2} A \nu-x A-\frac{A_{0}+A_{x}}{\nu}-\frac{\theta_{\infty}}{2} \frac{\sigma_{3}}{\nu(\nu-1)}\right] \Psi_{O U T}, \\
\frac{d \Psi_{I N}}{d \mu}=\left[x \frac{\theta_{\infty}}{2} \sigma_{3}+\frac{A_{0}}{\mu}+\frac{A_{x}}{\mu-1}\right] \Psi_{I N}=\left[x \frac{\theta_{\infty}}{2} \sigma_{3}+\frac{A_{0}+A_{x}}{\mu}-\frac{A_{0}}{\mu(\mu-1)}\right] \Psi_{I N} .
\end{gathered}
$$

Then, substitute $A_{0} \mapsto-A$ in the last term

We rewrite the systems at infinity ${ }^{6}$ :

$$
\begin{gathered}
\frac{d \tilde{\Psi}_{\text {OUT }}}{d \nu}=\left[x^{2} \frac{\theta_{x}}{2} \sigma_{3} \nu+x \frac{\theta_{x}}{2} \sigma_{3}-\frac{G^{-1}\left(A_{0}+A_{x}\right) G}{\nu}-\frac{\theta_{\infty}}{2} G^{-1} \sigma_{3} G\left(\frac{1}{\nu^{2}}+\frac{1}{\nu^{3}}+\ldots\right)\right] \tilde{\Psi}_{O U T} \\
\frac{d \Psi_{I N}}{d \mu}=\left[x \frac{\theta_{\infty}}{2} \sigma_{3}+\frac{A_{0}+A_{x}}{\mu}+A\left(\frac{1}{\mu^{2}}+\frac{1}{\mu^{3}}+\ldots\right)\right] \Psi_{I N}, \quad \nu, \mu \rightarrow \infty
\end{gathered}
$$

This time the system of $\tilde{\Psi}_{\text {OUT }}$ is in the form (63), while the system of $\Psi_{I N}$ is in the form (62), where:

$$
\begin{gathered}
\Omega=x \frac{\theta_{\infty}}{2} \sigma_{3}, \quad \Omega_{1}=\text { diagonal part of }\left(A_{0}+A_{x}\right), \\
\Lambda=\frac{\theta_{x}}{2} \sigma_{3}, \quad \Lambda_{1}=\text { diagonal part of }\left(-G^{-1}\left(A_{0}+A_{x}\right) G\right) .
\end{gathered}
$$

We impose that $\Omega_{1}$ and $\Lambda_{1}$ do not depend on $x$, and we get the conditions $a=0, c=s\left(s+\theta_{x}\right) b$. Then, we choose the following solutions:

$$
\begin{gathered}
\Psi_{\text {OUT }}=G\left[I+\frac{K_{1}}{\nu}+\ldots\right] \exp \left\{x^{2} \frac{\theta_{x}}{4} \sigma_{3} \nu^{2}+x \frac{\theta_{x}}{2} \sigma_{3} \nu\right\} G^{-1} \\
=I+\frac{\theta_{x}}{2} G \sigma_{3} G^{-1} \frac{x}{\lambda}+G K_{1} G^{-1} \lambda+O\left(\frac{x^{2}}{\lambda^{2}}, x, \lambda^{2}\right), \quad \nu \rightarrow \infty . \\
\Psi_{I N}=\left[I+\frac{G_{1}}{\mu}+\ldots\right] \exp \left\{x \frac{\theta_{\infty}}{2} \sigma_{3} \mu\right\}=I+G_{1} \frac{x}{\lambda}+\frac{\theta_{\infty}}{2} \sigma_{3} \lambda+O\left(\frac{x^{2}}{\lambda^{2}}, x, \lambda^{2}\right), \quad \mu \rightarrow \infty .
\end{gathered}
$$

\footnotetext{
${ }^{6}$ The system (67) is in the form of a system of isomonodromy deformation for the fifth Painleve equation.
} 
The relation of dominance among terms are considered as if the $G_{n}$ 's and $K_{n}$ 's do not diverge as $x \rightarrow 0$. The matching conditition:

$$
\Psi_{\text {OUT }}(\lambda, x) \sim \Psi_{I N}(\lambda, x), \quad x \rightarrow 0, \quad|x|^{\delta_{\text {OUT }}} \leq|\lambda| \leq|x|^{\delta_{I N}} .
$$

is restricted to the overlapping sector where both expansions of $\Psi_{O U T}$ and $\Psi_{I N}$ hold. We note that $\frac{x}{\lambda}$ vanishes slower than $\lambda$, because $\delta_{I N}>\frac{1}{2}$ (namely, $x / \lambda \sim x^{1-\delta}, \lambda \sim x^{\delta}, \delta>1 / 2$ ). $\Psi_{I N}$ and $\Psi_{\text {OUT }}$ automatically match in the first term $I$. We impose the matching of the second leading term, i.e. the term in $\frac{x}{\lambda}$ :

$$
G_{1}(x) \sim \frac{\theta_{x}}{2} G \sigma_{3} G^{-1} \equiv-A, \quad x \rightarrow 0 .
$$

As for $G_{1}$, the formulae in Appendix 2 give:

$$
\begin{gathered}
\left(G_{1}\right)_{i j}=-2 \frac{\left(A_{0}+A_{x}\right)_{i j}}{x \theta_{\infty}\left[\left(\sigma_{3}\right)_{i i}-\left(\sigma_{3}\right)_{j j}\right]}, \quad i \neq j . \\
\left(G_{1}\right)_{i i}=-(A)_{i i}+2 \frac{\left(A_{0}+A_{x}\right)_{i j}\left(A_{0}+A_{x}\right)_{j i}}{x \theta_{\infty}\left[\left(\sigma_{3}\right)_{j j}-\left(\sigma_{3}\right)_{i i}\right]} .
\end{gathered}
$$

In the last formula, $j=2$ if $i=1, j=1$ if $i=2$. Explicitly:

$$
\begin{gathered}
\left(G_{1}\right)_{12}=-\frac{r}{x \theta_{\infty}} b, \quad\left(G_{1}\right)_{21}=\frac{s\left(s+\theta_{x}\right)}{x \theta_{\infty} r} b, \\
\left(G_{1}\right)_{11}=-(A)_{11}-\frac{s\left(s+\theta_{x}\right)}{x \theta_{\infty}} b^{2}, \quad\left(G_{1}\right)_{22}=-(A)_{22}+\frac{s\left(s+\theta_{x}\right)}{x \theta_{\infty}} b^{2} .
\end{gathered}
$$

Therefore (69) $\Longleftrightarrow b(x) \sim-x \theta_{\infty}$, and $\sigma_{0}=1$. As it must be, we get the same result of the matching for the first choice.

\subsection{Critical Matching: $\frac{1}{2}-\epsilon<\delta_{I N} \leq \delta_{O U T}<\frac{1}{2}+\epsilon$.}

In between the first and the second choice - which hold respectively for $\frac{1}{3}<\delta_{I N} \leq \delta_{\text {OUT }}<\frac{1}{2}$ and $\frac{1}{2}<\delta_{I N} \leq \delta_{\text {OUT }}<\frac{2}{3}$ - we can also consider the following approximations of system (4):

$$
\begin{gathered}
\frac{d \Psi_{\text {OUT }}}{d \lambda}=\left[\frac{x A}{\lambda^{2}}+\frac{A_{0}+A_{x}}{\lambda}-\frac{\theta_{\infty}}{2} \frac{\sigma_{3}}{\lambda-1}\right] \Psi_{O U T}, \\
\frac{d \Psi_{I N}}{d \lambda}=\left[\frac{A_{0}}{\lambda}+\frac{A_{x}}{\lambda-x}+\frac{\theta_{\infty}}{2} \sigma_{3}\right] \Psi_{I N} .
\end{gathered}
$$

Rigorously speaking, the two systems cannot be considered simultaneously when $\sigma_{0}=1$. But we can consider $\sigma_{0}=1$ as a "limit" value - or "critical" value - for the matching of the two above systems in the region specified by $\frac{1}{2}-\epsilon<\delta_{I N} \leq \delta_{O U T}<\frac{1}{2}+\epsilon$, where $\epsilon>0$ is sufficiently small. We write again $\Psi_{O U T}=: G \tilde{\Psi}_{O U T}$. Then:

$$
\begin{gathered}
\frac{d \tilde{\Psi}_{\text {OUT }}}{d \nu}=\left[x \frac{\theta_{x}}{2} \sigma_{3}-\frac{G^{-1}\left(A_{0}+A_{x}\right) G}{\nu}-\frac{\theta_{\infty}}{2} G^{-1} \sigma_{3} G\left(\frac{1}{\nu^{2}}+\frac{1}{\nu^{3}}+\ldots\right)\right] \tilde{\Psi}_{\text {OUT }}, \quad \nu \rightarrow \infty \\
\frac{d \Psi_{I N}}{d \mu}=\left[x \frac{\theta_{\infty}}{2} \sigma_{3}+\frac{A_{0}+A_{x}}{\mu}+A\left(\frac{1}{\mu^{2}}+\frac{1}{\mu^{3}}+\ldots\right)\right] \Psi_{I N}, \quad \mu \rightarrow \infty .
\end{gathered}
$$

When we impose isomonodromicity conditions, the diagonal parts of $A_{0}+A_{x}$ and $G^{-1}\left(A_{0}+A_{x}\right) G$ must be independent of $x$. This gives again $a=0, c=s\left(s+\theta_{x}\right) b$. Then, we choose the fundamental solutions:

$$
\begin{aligned}
\Psi_{\text {OUT }}=G\left[I+\frac{G_{1}^{O U T}}{\nu}\right. & +\ldots] \quad \exp \left\{x \frac{\theta_{x}}{2} \sigma_{3} \nu\right\} G^{-1}= \\
& =I+G G_{1}^{O U T} G^{-1} \lambda+\frac{\theta_{x}}{2} G \sigma_{3} G^{-1} \frac{x}{\lambda}+O\left(\lambda^{2}, x, \frac{x^{2}}{\lambda^{2}}\right)
\end{aligned}
$$




$$
\Psi_{I N}=\left[I+G_{1}^{I N} \frac{x}{\lambda}+\ldots\right] \exp \left\{x \frac{\theta_{\infty}}{2} \mu\right\}=I+\frac{\theta_{\infty}}{2} \sigma_{3} \lambda+G_{1}^{I N} \frac{x}{\lambda}+O\left(\lambda^{2}, x, \frac{x^{2}}{\lambda^{2}}\right)
$$

We match them for $\lambda \sim x^{\delta}$ and $\delta \in(1 / 2-\epsilon, 1 / 2+\epsilon)$, in the overlapping sector where the above expansions hold. Here both $\lambda \sim x^{\delta}$ and $x / \lambda \sim x^{1-\delta}$ are the dominant terms. The matching conditions are $G G_{1}^{O U T} G^{-1} \sim \frac{\theta_{\infty}}{2} \sigma_{3}$ and $G_{1}^{I N} \sim \frac{\theta_{x}}{2} G \sigma_{3} G^{-1}$. Namely:

$$
G_{1}^{O U T}(x) \sim \frac{\theta_{\infty}}{2} G^{-1} \sigma_{3} G, \quad G_{1}^{I N}(x) \sim-A, \quad x \rightarrow 0
$$

The matrix $\frac{\theta_{\infty}}{2} G^{-1} \sigma_{3} G$ can be derived from (64). The matrix $G_{1}^{O U T}$ is (65), the matrix $G_{1}^{I N}$ is (70). Condition (71) is inclusive of both (66) and (69). Therefore, (71) $\Longleftrightarrow b(x) \sim-x \theta_{\infty}, x \rightarrow 0$. This is again the expected result.

\subsection{Higher Order Terms}

The final result obtained above is:

$$
\begin{gathered}
A_{0}+A_{x}=\left(\begin{array}{cc}
0 & -r \theta_{\infty} x \\
-\frac{\left(s+\theta_{x}\right) s \theta_{\infty}}{r} x & 0
\end{array}\right)+o(x), \quad A_{1}=-\frac{\theta_{\infty}}{2} \sigma_{3}-\left(A_{0}+A_{x}\right), \\
A_{x}=\left(\begin{array}{cc}
s+\frac{\theta_{x}}{2} & -r \\
\frac{\left(s+\theta_{x}\right) s}{r} & -s-\frac{\theta_{0}}{2}
\end{array}\right)+o(1), \quad A_{0}=-\left(\begin{array}{cc}
s+\frac{\theta_{x}}{2} & -r \\
\frac{\left(s+\theta_{x}\right) s}{r} & -s-\frac{\theta_{0}}{2}
\end{array}\right)+o(1)
\end{gathered}
$$

Let us substitute the above results into (6). We obtain the first term with no error:

$$
y(x) \sim \frac{1}{1-\theta_{\infty}}, \quad x \rightarrow 0 .
$$

Here, $r$ and $s$ do not appear. Nevertheless, if we substitute in (PVI) the series $y=\frac{1}{1-\theta_{\infty}}+\sum_{n=1}^{\infty} b_{n} x^{n}$, we can compute recursively all the terms, for $\theta_{0}= \pm \theta_{x}$ and $\theta_{1}= \pm \theta_{\infty}$. We find a series:

$$
y(x)=\frac{1}{1-\theta_{\infty}}+a x+\sum_{n=0}^{\infty} b_{n}\left(a ; \theta_{\infty}, \theta_{0}\right) x^{n}, \quad x \rightarrow 0,
$$

where $a$ is an arbitrary parameter. This parameter is actually a function of $s$, as we prove now. The convergence of the Taylor expansion can be proved by a Briot-Bouquet like argument. This will not be done here. The reader can find a similar proof in [19] and the general procedure in [14].

\subsubsection{Determination of $a=a(s)$}

The system (4) is isomonodromic. This determines the structure $A_{x}, A_{0} A_{1}$ as can be found in [18], Appendix C, formulae (C.47), (C.49), (C.51), (C.52), (C.55). If we substitute (74) in the formulae, we get a Taylor expansion for the matrix elements, in terms of the parameter $a$. The leading terms have exactly the structure of (72) and (73). We can identify the leading terms to express $a$ as a function of $s$ and $r$. The computations are quite long, so we give the result. When we write the leading terms as a function of $a$ and impose that they coincide with (72) and (73), we find:

$$
a=\frac{\theta_{\infty}\left(2 s+\theta_{x}+1\right)}{2\left(\theta_{\infty}-1\right)} \in \mathbf{C}
$$

The higher order terms are Taylor expansions. Explicitly, the first terms are:

$$
\begin{gathered}
A_{1}=-\frac{\theta_{\infty}}{2} \sigma_{3}-\left(A_{0}+A_{x}\right)= \\
=\left(\begin{array}{cc}
-\frac{\theta_{\infty}}{2}+\left(s+\theta_{x}\right) s \theta_{\infty} x^{2} & r \theta_{\infty}\left\{x-\frac{\left(\theta_{\infty}+1\right)\left(2 s+\theta_{x}-1\right)}{2} x^{2}\right\} \\
\frac{s \theta_{\infty}}{r}\left\{\left(\theta_{x}+s\right) x-\frac{\left(\theta_{\infty}-1\right)\left(\theta_{x}+s\right)\left(2 s+\theta_{x}+1\right) s}{2} x^{2}\right\} & \frac{\theta_{\infty}}{2}-\left(\theta_{x}+s\right) s \theta_{\infty} x^{2}
\end{array}\right)+O\left(x^{3}\right)
\end{gathered}
$$




$$
\begin{gathered}
A_{x}=\left(\begin{array}{cc}
\left(s+\frac{\theta_{x}}{2}\right)-2\left(s+\theta_{x}\right) s \theta_{\infty} x & -r\left\{1-\left(2 s+\theta_{x}-1\right) \theta_{\infty} x\right\} \\
\frac{s\left(s+\theta_{x}\right)}{r}\left\{1-\left(2 s+\theta_{x}+1\right) \theta_{\infty} x\right\} & -\left(s+\frac{\theta_{x}}{2}\right)+2\left(s+\theta_{x}\right) s \theta_{\infty} x
\end{array}\right)+O\left(x^{2}\right), \\
A_{0}=\left(\begin{array}{cc}
-\left(s+\frac{\theta_{x}}{2}\right)+2\left(s+\theta_{x}\right) s \theta_{\infty} x & r\left\{1-\left(2 s+\theta_{x}\right) \theta_{\infty} x\right\} \\
-\frac{s\left(s+\theta_{x}\right)}{r}\left\{1-\left(2 s+\theta_{x}\right) \theta_{\infty} x\right\} & \left(s+\frac{\theta_{x}}{2}\right)-2\left(s+\theta_{x}\right) s \theta_{\infty} x
\end{array}\right)+O\left(x^{2}\right) .
\end{gathered}
$$

The above expansions are enough to obtain first two leading terms of (6):

$$
y(x)=\frac{1}{1-\theta_{\infty}}+\frac{\theta_{\infty}\left(2 s+\theta_{x}+1\right)}{2\left(\theta_{\infty}-1\right)} x+O\left(x^{2}\right),
$$

Note that $r$ simplifies. This is the solution (19).

\subsection{Monodromy Data}

We assume that the matching has been completed as above, and in particular $\sigma_{0}=1$. Thus, the system (4) can be approximated by:

$$
\frac{d \Psi_{\text {OUT }}}{d \lambda}=\left[\frac{A_{0}+A_{x}}{\lambda}+\frac{x A_{x}}{\lambda^{2}}-\frac{\theta_{\infty}}{2} \frac{\sigma_{3}}{\lambda-1}\right] \Psi_{\text {OUT }}, \quad \text { for }|\lambda| \geq|x|^{\delta}, \quad \delta<\frac{1}{2} ;
$$

or

$$
\frac{d \Psi_{I N}}{d \lambda}=\left[\frac{A_{0}}{\lambda}+\frac{A_{x}}{\lambda}+\frac{\theta_{\infty}}{2} \sigma_{3}\right] \Psi_{I N}, \quad \text { for } \quad|\lambda| \leq|x|^{\delta}, \quad \delta>\frac{1}{2}
$$

The first two leading terms are :

$$
\begin{gathered}
\Psi_{\text {OUT }}=I+\frac{\theta_{\infty}}{2} \sigma_{3} \lambda+O\left(\frac{x}{\lambda}\right), \quad \lambda \sim x^{\delta} \rightarrow 0, \quad \delta<\frac{1}{2} ; \\
\Psi_{I N}=I-A \frac{x}{\lambda}+O(\lambda), \quad \lambda \sim x^{\delta} \rightarrow 0, \quad \delta>\frac{1}{2} .
\end{gathered}
$$

The above solutions match in the first three terms in the "critical" region $\lambda \sim x^{\delta}, \delta \simeq \frac{1}{2}$ (the region is restricted to a sector). Namely:

$$
\Psi_{\text {OUT }} \sim \Psi_{I N} \sim I+\frac{\theta_{\infty}}{2} \sigma_{3} \lambda-A \frac{x}{\lambda}+O\left(x, \frac{x^{2}}{\lambda^{2}}, \lambda^{2}\right), \quad \lambda \sim x^{\delta}, \quad \delta \simeq \frac{1}{2}
$$

Now, for $\delta<\frac{1}{2}$, we have:

$$
\frac{A_{0}+A_{x}}{\lambda} \sim x^{1-\delta} \rightarrow 0, \quad \frac{x A_{x}}{\lambda^{2}} \sim x^{1-2 \delta} \rightarrow 0 .
$$

Thus, (78) can be further reduced to:

$$
\frac{d \hat{\Psi}_{O U T}}{d \lambda}=-\frac{\theta_{\infty}}{2} \frac{\sigma_{3}}{\lambda-1} \hat{\Psi}_{O U T}
$$

In system (79) we rewrite:

$$
\frac{A_{0}}{\lambda}+\frac{A_{x}}{\lambda-x}=\frac{A_{0}+A_{x}}{\lambda-x}-\frac{x A_{0}}{\lambda(\lambda-x)}
$$

Then, we observe that, for $\delta>\frac{1}{2}$, we have the behaviors:

$$
\frac{A_{0}+A_{x}}{\lambda-x} \sim x^{1-\delta} \rightarrow 0, \quad \frac{x A_{0}}{\lambda(\lambda-x)} \sim-\frac{x A}{\lambda(\lambda-x)} \sim x^{1-2 \delta} \rightarrow \infty, \quad \delta>\frac{1}{2}
$$


Thus, as $x \rightarrow 0$, the system (79) can be further reduced to:

$$
\frac{d \hat{\Psi}_{I N}}{d \lambda}=\frac{x A}{\lambda(\lambda-x)} \hat{\Psi}_{I N} .
$$

System (83) has the same monodromy of (4) at $\lambda=1, \infty$. System (84) has the same monodromy of (4) at $\lambda=0, x$.

MATCHING $\Psi \leftrightarrow \hat{\Psi}_{O U T}$ :

We choose $\hat{\Psi}_{\text {OUT }}$ such that it matches with $\Psi$ at $\lambda=\infty$. The behavior of this last is:

$$
\Psi(\lambda, x)=\left[I+O\left(\frac{1}{\lambda}\right)\right] \lambda^{-\frac{\theta_{\infty}}{2} \sigma_{3}}, \quad \lambda \rightarrow \infty,
$$

for $\theta_{\infty} \notin \mathbf{Z}$. The solution of (83) with the same behavior is:

$$
\hat{\Psi}_{\text {OUT }}(\lambda):=(\lambda-1)^{-\frac{\theta_{\infty}}{2} \sigma_{3}}
$$

As a consequence, the monodromy of $\Psi$ at $\lambda=1, \infty$ coincides with that of $\hat{\Psi}_{\text {OUT }}$. To compute it, we consider the loops $\lambda-1 \mapsto(\lambda-1) e^{2 \pi i}$ and $\lambda \mapsto \lambda e^{2 \pi i}$. The corresponding monodromy is:

$$
M_{1}=M_{\infty}=\exp \left\{i \pi \theta_{\infty} \sigma_{3}\right\}
$$

MATCHING $\hat{\Psi}_{O U T} \leftrightarrow \Psi_{O U T}$ :

We match $\hat{\Psi}_{\text {OUT }}$ with $\Psi_{\text {OUT }}$ for $x / \lambda \rightarrow 0$. Let us choose the branch $(\lambda-1)=(1-\lambda) e^{i \pi}$, $(1-\lambda)>0$ for $0<\lambda<1$. Solution (85) has expansion:

$$
\hat{\Psi}_{\text {OUT }}(\lambda)=e^{-i \frac{\pi}{2} \theta_{\infty} \sigma_{3}}\left[I+\frac{\theta_{\infty}}{2} \sigma_{3} \lambda+O\left(\lambda^{2}\right)\right], \quad \lambda \rightarrow 0
$$

Therefore, for $\lambda \rightarrow 0, \hat{\Psi}_{\text {OUT }}$ matches with $\Psi_{\text {OUT }} e^{-i \frac{\pi}{2} \theta_{\infty} \sigma_{3}}$, where $\Psi_{\text {OUT }}$ is $(80)$.

MATCHING $\Psi_{O U T} \leftrightarrow \Psi_{I N}$ : This is (82).

MATCHING $\Psi_{I N} \leftrightarrow \Psi$ : The above matchings imply that $\Psi_{I N} e^{-i \frac{\pi}{2} \theta_{\infty} \sigma_{3}}$ matches with $\Psi$ (where $\Psi_{I N}$ is (81)).

MATCHING $\Psi_{I N} \leftrightarrow \hat{\Psi}_{I N}$ :

In order to determine the monodromy of system (4) at $\lambda=0, x$, we need to find a fundamental solution $\hat{\Psi}_{I N}$ of (84), that matches with $\Psi_{I N} e^{-i \frac{\pi}{2} \sigma_{3}}$ for $x / \lambda \rightarrow 0$, where $\Psi_{I N}$ is (81). A fundamental solution satisfying these requirements is:

$$
\hat{\Psi}_{I N}(\lambda, x):=G\left[\left(1-\frac{x}{\lambda}\right)^{-\frac{\theta_{x}}{2} \sigma_{3}}\right] G^{-1} e^{-i \frac{\pi}{2} \theta_{\infty} \sigma_{3}} .
$$

Actually, this has the behavior:

$$
\hat{\Psi}_{I N}(\lambda, x)=\left[I+\frac{\theta_{x}}{2} G \sigma_{3} G^{-1} \frac{x}{\lambda}+O\left(\frac{x^{2}}{\lambda^{2}}\right)\right] e^{-i \frac{\pi}{2} \theta_{\infty} \sigma_{3}}, \quad \frac{x}{\lambda} \rightarrow 0,
$$

where $\frac{\theta_{x}}{2} G \sigma_{3} G^{-1} \equiv-A$. The first two terms match with $\Psi_{I N} e^{-i \frac{\pi}{2} \theta_{\infty} \sigma_{3}}$, as required.

As a consequence of the matching, the monodromy matrices of $\Psi$ and $\hat{\Psi}_{I N}$ at $\lambda=0, x$ coincide. To compute them, we write the local behaviors (for $x \neq 0$ fixed) of $\hat{\Psi}_{I N}$ :

$$
\hat{\Psi}_{I N}(\lambda, x)=\left\{\begin{array}{c}
{\left[G x^{\frac{\theta_{x}}{2} \sigma_{3}}\right][1+O(\lambda-x)](\lambda-x)^{-\frac{\theta_{x}}{2} \sigma_{3}}\left[G^{-1} e^{-i \frac{\pi}{2} \theta_{\infty} \sigma_{3}}\right], \quad \lambda \rightarrow x,} \\
{\left[G(-x)^{-\frac{\theta_{x}}{2} \sigma_{3}}\right][1+O(\lambda)] \lambda^{\frac{\theta_{x}}{2} \sigma_{3}}\left[G^{-1} e^{-i \frac{\pi}{2} \theta_{\infty} \sigma_{3}}\right], \quad \lambda \rightarrow 0 .}
\end{array}\right.
$$


It is not necessary to specify the branch of $( \pm x)^{-\frac{\theta_{x}}{2} \sigma_{3}}$. We resctict to the case $\theta_{0}$ and $\theta_{x} \notin \mathbf{Z}$, so that the matrices $R_{0}, R_{x}$ in (17) are zero and the matching of $\Psi$ with the above behaviors of $\hat{\Psi}_{I N}$ is realized.

We can compute the monodromy matrices for $\lambda \mapsto \lambda e^{2 \pi i}$ and $(\lambda-x) \mapsto(\lambda-x) e^{2 \pi i}$ respectively:

$$
\begin{gathered}
M_{0}=e^{i \frac{\pi}{2} \theta_{\infty} \sigma_{3}} G \exp \left\{i \pi \theta_{x} \sigma_{3}\right\} G^{-1} e^{-i \frac{\pi}{2} \theta_{\infty} \sigma_{3}}, \\
M_{x}=e^{i \frac{\pi}{2} \theta_{\infty} \sigma_{3}} G \exp \left\{-i \pi \theta_{x} \sigma_{3}\right\} G^{-1} e^{-i \frac{\pi}{2} \theta_{\infty} \sigma_{3}},
\end{gathered}
$$

Note that $M_{1}=M_{\infty}$ is invariant for $M_{1} \mapsto e^{-i \frac{\pi}{2} \theta_{\infty} \sigma_{3}} M_{1} e^{i \frac{\pi}{2} \theta_{\infty} \sigma_{3}}$. With this fact in mind, we obtain the result of theorem 3, point b). In particular, computing the trace of $M_{0} M_{1}$, we get:

$$
s=\frac{\theta_{x}\left[2 \cos \left(\pi\left(\theta_{\infty}+\theta_{x}\right)\right)-\operatorname{tr}\left(M_{1} M_{0}\right)\right]}{2\left[\cos \left(\pi\left(\theta_{\infty}-\theta_{x}\right)\right)-\cos \left(\pi\left(\theta_{\infty}+\theta_{x}\right)\right)\right]} .
$$

\section{Case $\sigma= \pm\left(\theta_{1}-\theta_{\infty}\right), \quad \pm\left(\theta_{1}+\theta_{\infty}\right)$. Solution (18).}

This case shows new features, namely $r$ and $r_{1}$ may be functions of $x$. For $\sigma= \pm\left(\theta_{1}-\theta_{\infty}\right), \pm\left(\theta_{1}+\theta_{\infty}\right)$ the matrices (41) (42) become:

$$
\begin{array}{ccc}
\sigma= \pm\left(\theta_{1}-\theta_{\infty}\right): & \hat{A}_{1}=\left(\begin{array}{cc}
-\frac{\theta_{1}}{2} & -r_{1} \\
0 & \frac{\theta_{1}}{2}
\end{array}\right), & \hat{A}_{0}+\hat{A}_{x}=\left(\begin{array}{cc}
\frac{\theta_{1}-\theta_{\infty}}{2} & r_{1} \\
0 & -\frac{\theta_{1}-\theta_{\infty}}{2}
\end{array}\right) . \\
\sigma= \pm\left(\theta_{1}+\theta_{\infty}\right): & \hat{A}_{1}=\left(\begin{array}{cc}
\frac{\theta_{1}}{2} & -r_{1} \\
0 & -\frac{\theta_{1}}{2}
\end{array}\right), & \hat{A}_{0}+\hat{A}_{x}=\left(\begin{array}{cc}
-\frac{\theta_{1}+\theta_{\infty}}{2} & r_{1} \\
0 & \frac{\theta_{1}+\theta_{\infty}}{2}
\end{array}\right) .
\end{array}
$$

The transpose matrices may be considered (namely, re-define $r_{1} \mapsto-\frac{\left[\sigma^{2}-\left(\theta_{1}-\theta_{\infty}\right)^{2}\right]\left[\sigma^{2}-\left(\theta_{1}+\theta_{\infty}\right)^{2}\right]}{16 \theta_{\infty}^{2} r_{1}}$ ).

The matrices $\hat{\hat{A}}_{0}$ and $\hat{\hat{A}}_{x}$ are again $(47)$ and (48). For definiteness, we will consider the upper triangular matrices $\hat{A}_{1}$ and $\hat{A}_{0}+\hat{A}_{x}$, and the choice $\sigma=\theta_{1}-\theta_{\infty}$. We distinguish three cases:

(I) $r_{1}$ is a non zero constant: In this case we just repeat the general matching procedure and find $y(x)$ as in (51). $r \neq 0$ is constant.

(II) $r_{1}=0$ and $r$ constant. This is a very easy case, because $\hat{A_{1}}=-\frac{\theta_{1}}{2} \sigma_{3}, \hat{A_{0}}+\hat{A_{x}}=\frac{\theta_{1}-\theta_{\infty}}{2} \sigma_{3}$. Therefore a fundamental solution of $(40)$ is:

$$
\Psi_{\text {OUT }}(\lambda)=(\lambda-1)^{-\frac{\theta_{1}}{2} \sigma_{3}} \lambda^{\frac{\theta_{1}-\theta_{\infty}}{2} \sigma_{3}}(-1)^{-\frac{\theta_{1}}{2} \sigma_{3}}=\left[I+\frac{\theta_{1}}{2} \sigma_{3}+O\left(\lambda^{2}\right)\right] \lambda^{\frac{\theta_{1}-\theta_{\infty}}{2} \sigma_{3}}, \quad \lambda \rightarrow 0 .
$$

The solution of (46), with $r$ constant, is the same of the general case. The matching is possible as in the general case, with $K_{0}=\left(\begin{array}{cc}x^{\frac{\theta_{1}-\theta_{\infty}}{2}} & 0 \\ 0 & x^{\frac{\theta_{\infty}-\theta_{1}}{2}}\end{array}\right)$. Therefore:

$$
\hat{A_{0}}=\left(\begin{array}{cc}
\frac{\theta_{0}^{2}-\theta_{x}^{2}+\sigma^{2}}{4 \sigma} & r x^{\theta_{1}-\theta_{\infty}} \\
-\frac{\left[\sigma^{2}-\left(\theta_{0}-\theta_{x}\right)^{2}\right]\left[\sigma^{2}-\left(\theta_{0}+\theta_{x}\right)^{2}\right]}{16 \sigma^{2} r} x^{\theta_{\infty}-\theta_{1}} & -\frac{\theta_{0}^{2}-\theta_{x}^{2}+\sigma^{2}}{4 \sigma}
\end{array}\right) .
$$

We obtain: $y(x) \sim \frac{x\left(\hat{A}_{0}\right)_{12}}{x\left[\left(\hat{A}_{0}\right)_{12}+\left(\hat{A}_{1}\right)_{12}\right]-\left(\hat{A}_{1}\right)_{12}}=\frac{x r x^{\theta_{1}-\theta} \infty}{x\left[x^{\theta_{1}-\theta \infty}+0\right]-0}=1$. This is the singular solution $y(x) \equiv 1$.

The case $r_{1}=r_{1}(x) \rightarrow 0$ may give a non-singular $y(x)$, provided that also $r=r(x) \rightarrow 0$. This will be proved below.

(III) Case $r_{1}=r_{1}(x)$.

A priori, we don't know if it is possible to have an $x$-dependent $r_{1}$. This is not actually possible if we approximate (4) with the fuchsian system (40) (i.e. (13)). Actually, (40) is a system with reducible monodromy (upper triangular):

$$
\frac{d \Psi_{\text {OUT }}}{d \lambda}=\left\{\left(\begin{array}{cc}
\frac{\theta_{1}-\theta_{\infty}}{2} & r_{1} \\
0 & -\frac{\theta_{1}-\theta_{\infty}}{2}
\end{array}\right) \frac{1}{\lambda}+\left(\begin{array}{cc}
-\frac{\theta_{1}}{2} & -r_{1} \\
0 & \frac{\theta_{1}}{2}
\end{array}\right) \frac{1}{\lambda-1}\right\} \Psi_{O U T} .
$$


There is a fundamental solution (obtained by variation of parameters):

$$
\Psi_{\text {OUT }}=\left[\begin{array}{cc}
(1-\lambda)^{-\frac{\theta_{1}}{2}} & 0 \\
0 & (1-\lambda)^{\frac{\theta_{1}}{2}}
\end{array}\right]\left[\begin{array}{cc}
1 & \frac{r_{1} F\left(1-\theta_{1}, \theta_{\infty}-\theta_{1}, \theta_{\infty}-\theta_{1}+1 ; \lambda\right)}{\theta_{\infty}-\theta_{1}} \\
0 & 1
\end{array}\right]\left[\begin{array}{cc}
\lambda^{\frac{\theta_{1}-\theta_{\infty}}{2}} & 0 \\
0 & \lambda^{-\frac{\theta_{1}-\theta_{\infty}}{2}}
\end{array}\right] .
$$

Here, $F(\ldots)$ denotes a Gauss hyper-geometric function. This solution has a diagonal monondromy matrix at $\lambda=0$ and an upper triangular monodromy matrix at $\lambda=1$, with $r_{1}$ in the $(1,2)$ matrix element. Therefore, $r_{1}$ must be independent of the monodromy preserving deformation $x$. We are sent back to the case (I) of constant $r_{1}$.

The only possibility for $r_{1}$ to depend on $x$ is that the matrices $\hat{A}_{0}, \hat{A}_{x}$ have a behavior, for $x \rightarrow 0$, such that the system (4) is approximated by a system (11) with singularity of the second kind at $\lambda=0$. Namely, system (4) must be approximated at least by:

$$
\frac{d \Psi_{\text {OUT }}}{d \lambda}=\left\{\frac{x A_{x}}{\lambda^{2}}+\frac{A_{0}+A_{x}}{\lambda}+\frac{A_{1}}{\lambda-1}\right\} \Psi_{\text {OUT }}
$$

Hypothesis: We consider the case:

$$
x A_{x}=\left(\begin{array}{cc}
0 & \rho(x) \\
0 & 0
\end{array}\right)+\text { higher orders } \quad x \rightarrow 0 .
$$

In the above hypothesis, (87) is:

$$
\frac{d \Psi_{\text {OUT }}}{d \lambda}=\left\{\left(\begin{array}{cc}
0 & \rho(x) \\
0 & 0
\end{array}\right) \frac{1}{\lambda^{2}}+\left(\begin{array}{cc}
\frac{\theta_{1}-\theta_{\infty}}{2} & r_{1} \\
0 & \frac{\theta_{1}-\theta_{\infty}}{2}
\end{array}\right) \frac{1}{\lambda}+\left(\begin{array}{cc}
-\frac{\theta_{1}}{2} & -r_{1} \\
0 & \frac{\theta_{1}}{2}
\end{array}\right) \frac{1}{\lambda-1}\right\} \Psi_{\text {OUT }}
$$

Let us write a $\Psi_{O U T}$ as a vector $\left(\begin{array}{l}\psi_{1} \\ \psi_{2}\end{array}\right)$. The system becomes:

$$
\frac{d \psi_{1}}{d \lambda}=\left(\frac{\theta_{1}-\theta_{\infty}}{2 \lambda}-\frac{\theta_{1}}{2(\lambda-1)}\right) \psi_{1}+\left(\frac{r_{1}}{\lambda}+\frac{\rho}{\lambda^{2}}-\frac{r_{1}}{\lambda-1}\right) \psi_{2}, \quad \frac{d \psi_{2}}{d \lambda}=\left(\frac{\theta_{\infty}-\theta_{1}}{2 \lambda}+\frac{\theta_{1}}{2(\lambda-1)}\right) \psi_{2} .
$$

This system is solvable by variation of parameters. Let $C_{1}$ and $C_{2}$ be integration constants. The general solution is:

$$
\begin{gathered}
\psi_{2}(\lambda)=C_{2} \lambda^{\frac{\theta_{\infty}-\theta_{1}}{2}}(\lambda-1)^{\frac{\theta_{1}}{2}}, \\
\psi_{1}(\lambda)=C_{1} \lambda^{\frac{\theta_{1}-\theta_{\infty}}{2}}(\lambda-1)^{-\frac{\theta_{1}}{2}}+C_{2} e^{i \pi \theta_{1}}\left[\frac{\rho}{\theta_{\infty}-\theta_{1}-1} \frac{F\left(1-\theta_{1}, \theta_{\infty}-\theta_{1}-1, \theta_{\infty}-\theta_{1}: \lambda\right)}{\lambda}+\right. \\
\left.+\frac{r_{1}-\rho}{\theta_{\infty}-\theta_{1}} F\left(1-\theta_{1}, \theta_{\infty}-\theta_{1}, \theta_{\infty}-\theta_{1}+1 ; \lambda\right)\right] \lambda^{\frac{\theta_{\infty}-\theta_{1}}{2}}(\lambda-1)^{-\frac{\theta_{1}}{2}} .
\end{gathered}
$$

Here $F(\ldots)$ denotes a Gauss Hyper-geometric equation. The choice of the branch is such that for $0<\lambda<1$, we have $0<1-\lambda=e^{-i \pi}(\lambda-1)$.

In order to write the local behavior for $\lambda \rightarrow 0$, we expand the hypergemoetric functions and $(\lambda-1)^{ \pm \theta_{1} / 2}=\left(e^{i \pi}(1-\lambda)\right)^{ \pm \theta_{1} / 2}$ :

$$
\begin{gathered}
\psi_{1}=C_{1} e^{-i \frac{\pi}{2} \theta_{1}} \lambda^{\frac{\theta_{1}-\theta_{\infty}}{2}} \\
\left(1+\frac{\theta_{1}}{2} \lambda+O\left(\lambda^{2}\right)\right)+C_{2} e^{i \frac{\pi}{2} \theta_{1}} \lambda^{\frac{\theta_{\infty}-\theta_{1}}{2}}\left[-\frac{\rho}{1+\theta_{1}-\theta_{\infty}} \frac{1}{\lambda}+\right. \\
\left.+\frac{\rho \theta_{1}\left(\theta_{1}-\theta_{\infty}+2\right)-2 r_{1}\left(\theta_{1}-\theta_{\infty}+1\right)}{2\left(\theta_{1}-\theta_{\infty}\right)\left(\theta_{1}-\theta_{\infty}+1\right)}+O(\lambda)\right] \\
\psi_{2}=C_{2} e^{i \frac{\pi}{2} \theta_{1}}\left(1-\frac{\theta_{1}}{2} \lambda+O\left(\lambda^{2}\right)\right) \lambda^{\frac{\theta_{\infty}-\theta_{1}}{2}}
\end{gathered}
$$


Therefore, we can take as a fundamental solution the following matrix (choose $C_{1}=e^{i \frac{\pi}{2} \theta_{1}}, C_{2}=$ $\left.e^{-i \frac{\pi}{2} \theta_{1}}\right)$ :

$$
\begin{aligned}
& \Psi_{\text {OUT }}=\left[\begin{array}{cc}
\left(1+\frac{\theta_{1}}{2} \lambda+\ldots\right) \lambda^{\frac{\theta_{1}-\theta_{\infty}}{2}} & {\left[-\frac{\rho}{1+\theta_{1}-\theta_{\infty}} \frac{1}{\lambda}+\frac{\rho \theta_{1}\left(\theta_{1}-\theta_{\infty}+2\right)-2 r_{1}\left(\theta_{1}-\theta_{\infty}+1\right)}{2\left(\theta_{1}-\theta_{\infty}\right)\left(\theta_{1}-\theta_{\infty}+1\right)}+\ldots\right] \lambda^{\frac{\theta_{\infty}-\theta_{1}}{2}}} \\
0 & \left(1-\frac{\theta_{1}}{2} \lambda+\ldots\right) \lambda^{\frac{\theta_{\infty}-\theta_{1}}{2}}
\end{array}\right] \\
& =\left\{\left(\begin{array}{cc}
0 & -\frac{\rho}{1+\theta_{1}-\theta_{\infty}} \\
0 & 0
\end{array}\right) \frac{1}{\lambda}+\left(\begin{array}{cc}
1 & \frac{\rho \theta_{1}\left(\theta_{1}-\theta_{\infty}+2\right)-2 r_{1}\left(\theta_{1}-\theta_{\infty}+1\right)}{2\left(\theta_{1}-\theta_{\infty}\right)\left(\theta_{1}-\theta_{\infty}+1\right)} \\
0 & 1
\end{array}\right)+O(\lambda)\right\} \lambda^{\frac{\theta_{1}-\theta_{\infty}}{2} \sigma_{3}}
\end{aligned}
$$

\subsection{Matching}

The above solution must be matched with the solution of system (46), with $\sigma=\theta_{1}-\theta_{\infty}$.

$$
\Psi_{0}\left(\frac{\lambda}{x}\right)=\left[I+K_{1} \frac{x}{\lambda}+O\left(\frac{x^{2}}{\lambda^{2}}\right)\right]\left(\begin{array}{cc}
\left(\frac{\lambda}{x}\right)^{\frac{\theta_{1}-\theta_{\infty}}{2}} & 0 \\
0 & \left(\frac{\lambda}{x}\right)^{\frac{\theta_{\infty}-\theta_{1}}{2}}
\end{array}\right) .
$$

From a standard computation we find:

$$
K_{1}=\left(\begin{array}{cc}
\frac{\theta_{0}^{2}-\left(\theta_{x}-\sigma\right)^{2}}{4 \sigma} & \frac{r}{\sigma+1} \\
\frac{\left(\theta_{0}^{2}-\left(\theta_{x}-\sigma\right)^{2}\right)\left(\theta_{0}^{2}-\left(\theta_{x}+\sigma\right)^{2}\right)}{16 \sigma^{2}(\sigma-1) r} & \frac{\left(\theta_{x}+\sigma\right)^{2}-\theta_{0}^{2}}{4 \sigma(\sigma+1)}
\end{array}\right), \quad r \neq 0 .
$$

Note that $r$ in (48) is constant, because the monodromy of (46) depends on $r$.

The matching relation $\Psi_{O U T}(\lambda) \sim K_{0}(x) \Psi_{0}(\lambda / x)$, reads:

$$
\begin{aligned}
\left\{\left(\begin{array}{cc}
0 & -\frac{\rho}{1+\theta_{1}-\theta_{\infty}} \\
0 & 0
\end{array}\right) \frac{1}{\lambda}+\left(\begin{array}{cc}
1 & \frac{\rho \theta_{1}\left(\theta_{1}-\theta_{\infty}+2\right)-2 r_{1}\left(\theta_{1}-\theta_{\infty}+1\right)}{2\left(\theta_{1}-\theta_{\infty}\right)\left(\theta_{1}-\theta_{\infty}+1\right)} \\
0 & 1
\end{array}\right)+O(\lambda)\right\} & \sim \\
& \sim K_{0}(x)\left[I+K_{1} \frac{x}{\lambda}+\ldots\right]\left(\begin{array}{cc}
x^{\frac{\theta_{\infty}-\theta_{1}}{2}} & 0 \\
0 & x^{\frac{\theta_{1}-\theta_{\infty}}{2}}
\end{array}\right) .
\end{aligned}
$$

Namely:

$$
\left\{\begin{array}{c}
\left(\begin{array}{cc}
1 & \frac{\rho \theta_{1}\left(\theta_{1}-\theta_{\infty}+2\right)-2 r_{1}\left(\theta_{1}-\theta_{\infty}+1\right)}{2\left(\theta_{1}-\theta_{\infty}\right)\left(\theta_{1}-\theta_{\infty}+1\right)} \\
0 & 1
\end{array}\right) \sim K_{0}(x)\left(\begin{array}{cc}
x^{\frac{\theta_{\infty}-\theta_{1}}{2}} & 0 \\
0 & x^{\frac{\theta_{1}-\theta_{\infty}}{2}}
\end{array}\right) \\
\left(\begin{array}{cc}
0 & -\frac{\rho}{1+\theta_{1}-\theta_{\infty}} \\
0 & 0
\end{array}\right) \sim x K_{0}(x) F_{1}^{(0)}\left(\begin{array}{cc}
x^{\frac{\theta_{\infty}-\theta_{1}}{2}} & x^{\frac{\theta_{1}-\theta_{\infty}}{2}} \\
0 &
\end{array}\right)
\end{array}\right.
$$

The first equation above is:

$$
K_{0}(x) \sim\left(\begin{array}{cc}
1 & \frac{\rho \theta_{1}\left(\theta_{1}-\theta_{\infty}+2\right)-2 r_{1}\left(\theta_{1}-\theta_{\infty}+1\right)}{2\left(\theta_{1}-\theta_{\infty}\right)\left(\theta_{1}-\theta_{\infty}+1\right)} \\
0 & 1
\end{array}\right)\left(\begin{array}{cc}
x^{\frac{\theta_{1}-\theta_{\infty}}{2}} & 0 \\
0 & x^{\frac{\theta_{\infty}-\theta_{1}}{2}}
\end{array}\right) .
$$

We substitute this in the second equation. For simplicity, denote $K_{i j}$ the matrix elements of $K_{1}$. We obtain:

$$
\left(\begin{array}{cc}
0 & -\frac{\rho}{1+\theta_{1}-\theta_{\infty}} \\
0 & 0
\end{array}\right) \sim\left(\begin{array}{cc}
x K_{11}+(*) K_{21} x^{\theta_{\infty}-\theta_{1}+1} & K_{12} x^{\theta_{1}-\theta_{\infty}+1}+(*) x K_{22} \\
K_{21} x^{\theta_{\infty}-\theta_{1}+1} & x K_{22}
\end{array}\right),
$$

where:

$$
(*):=\frac{\rho \theta_{1}\left(\theta_{1}-\theta_{\infty}+2\right)-2 r_{1}\left(\theta_{1}-\theta_{\infty}+1\right)}{2\left(\theta_{1}-\theta_{\infty}\right)\left(\theta_{1}-\theta_{\infty}+1\right)}
$$


The element $(2,1)$ must vanish. This occurs iff:

$$
x^{\theta_{\infty}-\theta_{1}+1} \rightarrow 0, \quad \text { for } x \rightarrow 0 ; \quad \Longleftrightarrow \Re\left(\theta_{\infty}-\theta_{1}\right)>-1 .
$$

We substitute this result in the element $(1,1)$ and then we impose that it vanishes:

$$
\frac{\rho \theta_{1}\left(\theta_{1}-\theta_{\infty}+2\right)-2 r_{1}\left(\theta_{1}-\theta_{\infty}+1\right)}{2\left(\theta_{1}-\theta_{\infty}\right)\left(\theta_{1}-\theta_{\infty}+1\right)} K_{21} x^{\theta_{\infty}-\theta_{1}+1} \rightarrow 0 .
$$

This implies that $\rho \theta_{1}\left(\theta_{1}-\theta_{\infty}+2\right)-2 r_{1}\left(\theta_{1}-\theta_{\infty}+1\right)=o\left(x^{\theta_{1}-\theta_{\infty}-1}\right)$.

From the element $(1,2)$ we have:

$$
-\frac{\rho}{1+\theta_{1}-\theta_{\infty}} \sim K_{12} x^{\theta_{1}-\theta_{\infty}+1}+o\left(x^{\theta_{1}-\theta_{\infty}}\right) K_{22} .
$$

This relation may be satisfied in two ways: the first is that $\rho=\rho(x)=o\left(x^{\theta_{1}-\theta_{\infty}}\right)$. The second is:

$$
\rho=\rho(x) \sim-\left(1+\theta_{1}-\theta_{\infty}\right) K_{12} x^{\theta_{1}-\theta_{\infty}+1}=-r x^{\theta_{1}-\theta_{\infty}+1} .
$$

In both cases $\rho$ is a function of $x$. We are going to prove that the monodromy of $\Psi_{\text {OUT }}$ is independent of $\rho(x)$ (namely, of $x$ ) if and only if:

$$
\rho=r_{1} \frac{\theta_{1}-\theta_{\infty}+1}{\theta_{1}}
$$

This fact rules out the first possibility, because (92) becomes:

$$
\frac{\rho(x)}{\theta_{\infty}-\theta_{1}-1} \sim K_{12} x^{\theta_{1}-\theta_{\infty}+1}+\text { constant } \times \rho(x) x,
$$

so the last term in the r.h.s. is a higher order correction and $\rho$ is given by (93). Before proving (94), we complete the matching procedure. Using (94), we find:

$$
K_{0}(x) \sim\left(\begin{array}{cc}
1 & g x^{\theta_{1}-\theta_{\infty}+1} \\
0 & 1
\end{array}\right)\left(\begin{array}{cc}
x^{\frac{\theta_{1}-\theta_{\infty}}{2}} & 0 \\
0 & x^{\frac{\theta_{\infty}-\theta_{1}}{2}}
\end{array}\right), \quad g:=-\frac{r}{\theta_{1}-\theta_{\infty}+1}
$$

We are ready to compute $\hat{A_{0}}=K_{0} \hat{\hat{A}}_{0} K_{0}{ }^{-1}$, where $\hat{\hat{A}_{0}}$ is $(47)$, for $\sigma=\theta_{1}-\theta_{\infty}$ :

$$
\hat{A_{0}}=\left(\begin{array}{cc}
\left(\hat{\hat{A}}_{0}\right)_{11}+g\left(\hat{\hat{A}}_{0}\right)_{21} x & \left(\frac{\left(\hat{\hat{A}}_{0}\right)_{12}}{x}-2 g\left(\hat{\hat{A}}_{0}\right)_{11}-g^{2}\left(\hat{\hat{A}}_{0}\right)_{21} x\right) x^{\theta_{1}-\theta_{\infty}+1} \\
\left(\left(\hat{\hat{A}_{0}}\right)_{21} x\right) \frac{1}{x^{\theta_{1}-\theta_{\infty}+1}} & -\left(\hat{\hat{A}_{0}}\right)_{11}-g\left(\hat{\hat{A}}_{0}\right)_{21} x
\end{array}\right) .
$$

The first term of each matrix element certainly contains no error.

We can now substitute $\left(\hat{A_{0}}\right)_{12}=\left(-\frac{r}{x}+O(1)\right) x^{\theta_{1}-\theta_{\infty}+1}$ and $\hat{A_{1}}=-r_{1}(x) \sim \frac{\theta_{1}}{\theta_{1}-\theta_{\infty}+1} r x^{\theta_{1}-\theta_{\infty}+1}$ into (6), and find:

$$
y(x) \sim \frac{\theta_{1}-\theta_{\infty}+1}{1-\theta_{\infty}}, \quad x \rightarrow 0 .
$$

Proof of (94). We compute the monodromy of the solution $\Psi_{\text {OUT }}$. At $\lambda=0$ this is given by the matrix $M_{0}^{O U T}=\exp \left\{i \pi\left(\theta_{1}-\theta_{\infty}\right) \sigma_{3}\right\}$, obtained by the expansion of $\Psi_{\text {OUT }}$ at $\lambda=0$ as we did above, with the choice $C_{1}=e^{i \frac{\pi}{2} \theta_{1}}=1 / C_{2}$.

Let us study the monodromy at $\lambda=1$. We need to expand (89) and (90) at $\lambda=1$. First of all, we use the contiguity relation:

$$
F\left(1-\theta_{1}, \theta_{\infty}-\theta_{1}, \theta_{\infty}-\theta_{1}+1 ; \lambda\right)=\frac{\theta_{1}-\theta_{\infty}}{\theta_{\infty}-1} \frac{1}{\lambda}\left[(1-\lambda)^{\theta_{1}}-F\left(1-\theta_{1}, \theta_{\infty}-\theta_{1}-1, \theta_{\infty}-\theta_{1} ; \lambda\right)\right] .
$$

This is used to rewrite $\psi_{1}$ :

$$
\psi_{1}=C_{1} \lambda^{\frac{\theta_{1}-\theta_{\infty}}{2}}(\lambda-1)^{-\frac{\theta_{1}}{2}}+C_{2} e^{i \pi \theta_{1}} \lambda^{\frac{\theta_{\infty}-\theta_{1}}{2}}(\lambda-1)^{-\frac{\theta_{1}}{2}}\left[\frac{\rho-r_{1}}{\theta_{\infty}-1} \frac{(1-\lambda)^{\theta_{1}}}{\lambda}+\right.
$$




$$
\left.+\left(\frac{\rho}{\theta_{\infty}-\theta_{1}-1}+\frac{r_{1}-\rho}{\theta_{\infty}-1}\right) \frac{1}{\lambda} F\left(1-\theta_{1}, \theta_{\infty}-\theta_{1}-1, \theta_{\infty}-\theta_{1} ; \lambda\right)\right]
$$

Then, we substitute in $\psi_{1}$ the following connection formula:

$$
\begin{array}{r}
F\left(1-\theta_{1}, \theta_{\infty}-\theta_{1}-1, \theta_{\infty}-\theta_{1} ; \lambda\right)=\frac{\Gamma\left(\theta_{1}\right) \Gamma\left(\theta_{\infty}-\theta_{1}\right)}{\Gamma\left(\theta_{\infty}-1\right)} F\left(1-\theta_{1}, \theta_{\infty}-\theta_{1}-1,1-\theta_{1} ; 1-\lambda\right)+ \\
+\frac{\Gamma\left(-\theta_{1}\right) \Gamma\left(\theta_{\infty}-\theta_{1}\right)}{\Gamma\left(1-\theta_{1}\right) \Gamma\left(\theta_{\infty}-\theta_{1}-1\right)}(1-\lambda)^{\theta_{1}} F\left(1, \theta_{\infty}-1,1+\theta_{1}: 1-\lambda\right), \quad \theta_{1}, \theta_{\infty} \notin \mathbf{Z} .
\end{array}
$$

Thus, $\psi_{1}$ has the following structure, when $\lambda \rightarrow 1$ :

$$
\begin{gathered}
\psi_{1}=C_{1} e^{-i \frac{\pi}{2} \theta_{1}} \lambda^{\frac{\theta_{1}-\theta_{\infty}}{2}}(1-\lambda)^{-\frac{\theta_{1}}{2}}+C_{2} e^{i \frac{\pi}{2} \theta_{1}} \lambda^{\frac{\theta_{\infty}-\theta_{1}}{2}}\left[(1-\lambda)^{\frac{\theta_{1}}{2}} \sum_{n=0}^{\infty} a_{n}(1-\lambda)^{n}+\right. \\
\left.+\left(\frac{\rho}{\theta_{\infty}-\theta_{1}-1}+\frac{r_{1}-\rho}{\theta_{\infty}-1}\right)(1-\lambda)^{-\frac{\theta_{1}}{2}} \sum_{n=0}^{\infty} b_{n}(1-\lambda)^{n}\right]
\end{gathered}
$$

where $a_{n}, b_{n} \neq 0$ are the coefficients that follow from the expansion $1 / \lambda$ and the hyper-geometric functions at $\lambda=1$. When $\lambda^{ \pm \frac{\theta_{\infty}-\theta_{1}}{2}}$ is also expanded at $\lambda=1$, we find that $\Psi_{\text {OUT }}$ has the following structure:

$$
\Psi_{\text {OUT }}=\left(\begin{array}{cc}
\left(\operatorname{series}_{1}\right)(1-\lambda)^{-\frac{\theta_{1}}{2}} & \left(\operatorname{series}_{2}\right)(1-\lambda)^{\frac{\theta_{1}}{2}}+ \\
0 & \left(\frac{\rho}{\theta_{\infty}-\theta_{1}-1}+\frac{r_{1}-\rho}{\theta_{\infty}-1}\right)\left(\operatorname{series}_{3}\right)(1-\lambda)^{-\frac{\theta_{1}}{2}} \\
\left(\operatorname{series}_{4}\right)(1-\lambda)^{\frac{\theta_{1}}{2}}
\end{array}\right) .
$$

Here "series" means a series of the form $\sum_{n=0}^{\infty} c_{n}(1-\lambda)^{n}$, with $c_{0} \neq 0$. We just give the dominant term, with the choice $C_{1}=e^{i \frac{\pi}{2} \theta_{1}}=1 / C_{2}$ :

$$
\begin{gathered}
\Psi_{O U T}=[I+O(1-\lambda)] \times \\
\times\left(\begin{array}{cc}
(1-\lambda)^{-\frac{\theta_{1}}{2}} & {\left[\frac{\rho-r_{1}}{\theta_{\infty}-1}+(* *) \frac{\Gamma\left(-\theta_{1}\right) \Gamma\left(\theta_{\infty}-\theta_{1}\right)}{\Gamma\left(1-\theta_{1}\right) \Gamma\left(\theta_{\infty}-\theta_{1}-1\right)}\right](1-\lambda)^{\frac{\theta_{1}}{2}}+(* *) \frac{\Gamma\left(\theta_{1}\right) \Gamma\left(\theta_{\infty}-\theta_{1}\right)}{\Gamma\left(\theta_{\infty}-1\right)}(1-\lambda)^{-\frac{\theta_{1}}{2}}} \\
0 & (1-\lambda)^{\frac{\theta_{1}}{2}}
\end{array}\right),
\end{gathered}
$$

where:

$$
(* *)=\frac{\rho}{\theta_{\infty}-\theta_{1}-1}+\frac{r_{1}-\rho}{\theta_{\infty}-1} .
$$

Let $v, w$ be non zero arbitrary numbers. We conclude that:

$$
\begin{aligned}
& \Psi_{\text {OUT }}=\left(\begin{array}{cc}
v & w \\
0 & w\left[\frac{\rho-r_{1}}{\theta_{\infty}-1}+(* *) \frac{\Gamma\left(-\theta_{1}\right) \Gamma\left(\theta_{\infty}-\theta_{1}\right)}{\Gamma\left(1-\theta_{1}\right) \Gamma\left(\theta_{\infty}-\theta_{1}-1\right)}\right]^{-1}
\end{array}\right)[I+O(1-\lambda)] \times \\
& \times\left(\begin{array}{cc}
(1-\lambda)^{-\frac{\theta_{1}}{2}} & 0 \\
0 & (1-\lambda)^{\frac{\theta_{1}}{2}}
\end{array}\right)\left(\begin{array}{cc}
\frac{1}{v} & \frac{1}{v}(* *) \frac{\Gamma\left(\theta_{1}\right) \Gamma\left(\theta_{\infty}-\theta_{1}\right)}{\Gamma\left(\theta_{\infty}-1\right)} \\
0 & \frac{1}{w}\left[\frac{\rho-r_{1}}{\theta_{\infty}-1}+(* *) \frac{\Gamma\left(-\theta_{1}\right) \Gamma\left(\theta_{\infty}-\theta_{1}\right)}{\Gamma\left(1-\theta_{1}\right) \Gamma\left(\theta_{\infty}-\theta_{1}-1\right)}\right]
\end{array}\right)
\end{aligned}
$$

The monodromy matrix is then:

$$
M_{1}^{\text {OUT }}=Q^{-1} e^{-i \pi \theta_{1} \sigma_{3}} Q, \quad Q:=\left(\begin{array}{cc}
\frac{1}{v} & \frac{1}{v}(* *) \frac{\Gamma\left(\theta_{1}\right) \Gamma\left(\theta_{\infty}-\theta_{1}\right)}{\Gamma\left(\theta_{\infty}-1\right)} \\
0 & \frac{1}{w}\left[\frac{\rho-r_{1}}{\theta_{\infty}-1}+(* *) \frac{\Gamma\left(-\theta_{1}\right) \Gamma\left(\theta_{\infty}-\theta_{1}\right)}{\Gamma\left(1-\theta_{1}\right) \Gamma\left(\theta_{\infty}-\theta_{1}-1\right)}\right]
\end{array}\right) .
$$

This is independent of $\rho(x)$ if and only if $(* *)=0$. This proves $(94)$.

(94) simplifies considerably the structure of (90):

$$
\psi_{1}=C_{1} \lambda^{\frac{\theta_{1}-\theta_{\infty}}{2}}(\lambda-1)^{-\frac{\theta_{1}}{2}}-C_{2} \frac{r_{1}}{\theta_{1}} \lambda^{\frac{\theta_{\infty}-\theta_{1}}{2}-1}(\lambda-1)^{\frac{\theta_{1}}{2}} .
$$


With the choice $C_{1}=\exp \left\{i \frac{\pi}{2} \theta_{1}\right\}=1 / C_{2}$, we finally have:

$$
\Psi_{\text {OUT }}=\left(\begin{array}{cc}
\lambda^{\frac{\theta_{1}-\theta_{\infty}}{2}}(1-\lambda)^{-\frac{\theta_{1}}{2}} & -\frac{r_{1}(x)}{\theta_{1}} \lambda^{\frac{\theta_{\infty}-\theta_{1}}{2}-1}(1-\lambda)^{\frac{\theta_{1}}{2}} \\
0 & \lambda^{\frac{\theta_{\infty}-\theta_{1}}{2}}(1-\lambda)^{\frac{\theta_{1}}{2}}
\end{array}\right) .
$$

Note: The monodromy at $\lambda=0,1$ is diagonal: $M_{0}^{O U T}=\exp \left\{i \pi\left(\theta_{1}-\theta_{\infty}\right) \sigma_{3}\right\}, M_{1}^{\text {OUT }}=\exp \left\{-i \pi \theta_{1} \sigma_{3}\right\}$. It is independent of $r_{1}$. In case (I) - namely, $r_{1}$ is a non-zero constant and the system for $\Psi_{1}$ is fuchsian - if $M_{0}^{O U T}$ is in diagonal form, then $M_{1}^{O U T}$ is upper triangular and depends on $r_{1}$.

\subsection{Higher Orders Terms}

We may repeat the same procedure of section 5.6. We write a Taylor expansion $y(x)=\left(\theta_{1}-\theta_{\infty}+\right.$ $1) /\left(1-\theta_{\infty}\right)+\sum_{n \geq 1} b_{n} x^{n}$, substitute it into (PVI) and determine recursively the $b_{n}$ 's. Then, we may substitute the result in the matrix elements of $A_{i}, i=0, x, 1$, according to the formulae of [18], and find the higher orders of the matrix elements. We just give the result.

$$
\begin{aligned}
& \left(A_{x}\right)_{11}=-\left(A_{x}\right)_{22}=\frac{\left(\theta_{\infty}-\theta_{1}\right)^{2}+\theta_{x}^{2}-\theta_{0}^{2}}{4\left(\theta_{1}-\theta_{\infty}\right)}+ \\
& +\frac{\theta_{1}}{8} \frac{\left[\left(\theta_{1}-\theta_{\infty}\right)^{2}-\left(\theta_{0}-\theta_{x}\right)^{2}\right]\left[\left(\theta_{1}-\theta_{\infty}\right)^{2}-\left(\theta_{0}+\theta_{x}\right)^{2}\right]}{\left(\theta_{1}-\theta_{\infty}\right)^{2}\left[\left(\theta_{1}-\theta_{\infty}\right)^{2}-1\right]} x+O\left(x^{2}\right) . \\
& \left(A_{x}\right)_{12}=-r\left\{\frac{1}{x}-\frac{\theta_{1}\left[\left(\theta_{1}-\theta_{\infty}+2\right)\left(\theta_{1}-\theta_{\infty}\right)+\theta_{0}^{2}-\theta_{x}^{2}\right]}{2\left(\theta_{1}-\theta_{\infty}\right)\left(\theta_{\infty}-\theta_{1}-1\right)}+O(x)\right\} x^{\theta_{1}-\theta_{\infty}+1} . \\
& \left(A_{x}\right)_{21}=\frac{1}{r} \frac{\left[\left(\theta_{1}-\theta_{\infty}\right)^{2}-\left(\theta_{0}-\theta_{x}\right)^{2}\right]\left[\left(\theta_{1}-\theta_{\infty}\right)^{2}-\left(\theta_{0}+\theta_{x}\right)^{2}\right]}{16\left(\theta_{\infty}-\theta_{1}\right)^{2}}\{x+ \\
& \left.-\frac{\theta_{1}\left[\left(\theta_{\infty}-\theta_{1}\right)\left(\theta_{\infty}-\theta_{1}+2\right)+\theta_{0}^{2}-\theta_{x}^{2}\right]}{2\left(\theta_{\infty}-\theta_{1}\right)^{3}\left(\theta_{\infty}-\theta_{1}+1\right)} x^{2}+O\left(x^{3}\right)\right\} \frac{1}{x^{\theta_{1}-\theta_{\infty}+1}} . \\
& \left(A_{0}\right)_{11}=-\left(A_{0}\right)_{22}=\frac{\left(\theta_{\infty}-\theta_{1}\right)^{2}+\theta_{0}^{2}-\theta_{x}^{2}}{4\left(\theta_{1}-\theta_{\infty}\right)}+ \\
& -\frac{\theta_{1}}{8} \frac{\left[\left(\theta_{1}-\theta_{\infty}\right)^{2}-\left(\theta_{0}-\theta_{x}\right)^{2}\right]\left[\left(\theta_{1}-\theta_{\infty}\right)^{2}-\left(\theta_{0}+\theta_{x}\right)^{2}\right]}{\left(\theta_{1}-\theta_{\infty}\right)^{2}\left[\left(\theta_{1}-\theta_{\infty}\right)^{2}-1\right]} x+O\left(x^{2}\right) . \\
& \left(A_{0}\right)_{12}=r\left\{\frac{1}{x}-\frac{\theta_{1}\left[\left(\theta_{1}-\theta_{\infty}\right)^{2}+\theta_{0}^{2}-\theta_{x}^{2}\right]}{2\left(\theta_{1}-\theta_{\infty}\right)\left(\theta_{\infty}-\theta_{1}-1\right)}+O(x)\right\} x^{\theta_{1}-\theta_{\infty}+1} . \\
& \left(A_{0}\right)_{21}=-\frac{1}{r} \frac{\left[\left(\theta_{1}-\theta_{\infty}\right)^{2}-\left(\theta_{0}-\theta_{x}\right)^{2}\right]\left[\left(\theta_{1}-\theta_{\infty}\right)^{2}-\left(\theta_{0}+\theta_{x}\right)^{2}\right]}{16\left(\theta_{\infty}-\theta_{1}\right)^{2}}\{x+ \\
& \left.-\frac{\theta_{1}\left[\left(\theta_{1}-\theta_{\infty}\right)^{2}+\theta_{0}^{2}-\theta_{x}^{2}\right]}{2\left(\theta_{\infty}-\theta_{1}\right)^{3}\left(\theta_{\infty}-\theta_{1}+1\right)} x^{2}+O\left(x^{3}\right)\right\} \frac{1}{x^{\theta_{1}-\theta_{\infty}+1}} . \\
& \left(A_{1}\right)_{11}=-\left(A_{1}\right)_{22}=-\frac{\theta_{1}}{2}-\frac{\theta_{1}\left[\left(\theta_{1}-\theta_{\infty}\right)^{2}-\left(\theta_{0}-\theta_{x}\right)^{2}\right]\left[\left(\theta_{1}-\theta_{\infty}\right)^{2}-\left(\theta_{0}+\theta_{x}\right)^{2}\right]}{16\left[\left(\theta_{\infty}-\theta_{1}\right)^{2}-1\right]\left(\theta_{\infty}-\theta_{1}\right)^{2}} x^{2}+O\left(x^{3}\right) . \\
& \left(A_{1}\right)_{12}=-r \frac{\theta_{1}}{\theta_{\infty}-\theta_{1}-1}\left\{1+\frac{\left(\theta_{1}+1\right)\left[\left(\theta_{\infty}-\theta_{1}\right)\left(\theta_{\infty}-\theta_{1}-2\right)+\theta_{0}^{2}-\theta_{x}^{2}\right]}{2\left(\theta_{\infty}-\theta_{1}\right)\left(\theta_{\infty}-\theta_{1}-2\right)} x+O\left(x^{2}\right)\right\} x^{\theta_{1}-\theta_{\infty}+1} . \\
& \left(A_{1}\right)_{21}=\left\{\frac{\theta_{1}\left[\left(\theta_{1}-\theta_{\infty}\right)^{2}-\left(\theta_{0}-\theta_{x}\right)^{2}\right]\left[\left(\theta_{1}-\theta_{\infty}\right)^{2}-\left(\theta_{0}+\theta_{x}\right)^{2}\right]}{16 r\left(\theta_{\infty}-\theta_{1}+1\right)\left(\theta_{\infty}-\theta_{1}\right)^{2}} x^{2}\right\} \frac{1}{x^{\theta_{1}-\theta_{\infty}+1}} .
\end{aligned}
$$

The above leading terms of $y(x)$ are related to the above formulae through (6). The truncation of $\left(A_{0}\right)_{12}$ and $\left(A_{1}\right)_{12}$ above is enough to reproduce the first two terms of solution (18):

$$
y(x)=\frac{\theta_{1}-\theta_{\infty}+1}{1-\theta_{\infty}}+\frac{\theta_{1}\left[\left(\theta_{1}-\theta_{\infty}\right)^{2}+\theta_{x}^{2}-\theta_{0}^{2}+2 \theta_{1}-2 \theta_{\infty}\right]}{2\left(1-\theta_{\infty}\right)\left(\theta_{\infty}-\theta_{1}\right)\left(\theta_{1}-\theta_{\infty}+2\right)} x+O\left(x^{2}\right) .
$$




\subsection{Transpose Case and General Result}

In the above computations, the condition $x^{\theta_{\infty}-\theta_{1}+1} \rightarrow 0$ was necessary to do the matching. We can repeat the matching procedure starting from the transpose matrices:

$$
\hat{A}_{1}=\left(\begin{array}{cc}
-\frac{\theta_{1}}{2} & 0 \\
-r_{1} & \frac{\theta_{1}}{2}
\end{array}\right), \quad \hat{A}_{0}+\hat{A}_{x}=\left(\begin{array}{cc}
\frac{\theta_{1}-\theta_{\infty}}{2} & 0 \\
r_{1} & -\frac{\theta_{1}-\theta_{\infty}}{2}
\end{array}\right), \quad x A_{x}=\left(\begin{array}{cc}
0 & 0 \\
\rho(x) & 0
\end{array}\right) .
$$

The procedure is exactly the same, with the necessary condition:

$$
x^{\theta_{1}-\theta_{\infty}+1} \rightarrow 0, \quad \text { when } x \rightarrow 0 .
$$

As a result we obtain again exactly the matrices $A_{0}, A_{x}, A_{1}$ above. The reader can convince himself without doing any computation, simply looking at the structure of the first terms of above matrices. For example, let us have a look at $A_{x}$. Denote the constant terms with letters $c_{1}, c_{2}, \ldots$, etc. We have:

$$
\begin{gathered}
A_{x}=\left(\begin{array}{cc}
c_{1}+\ldots & \left\{\frac{c_{2}}{x}+\ldots\right\} x^{\theta_{1}-\theta_{\infty}+1} \\
\left\{c_{3} x+\ldots\right\} \frac{1}{x^{\theta_{1}-\theta_{\infty}+1}} & -c_{1}+\ldots
\end{array}\right)= \\
=\left(\begin{array}{cc}
c_{1}+\ldots & \left\{c_{2} x+\ldots\right\} \frac{1}{x^{\theta_{\infty}-\theta_{1}+1}} \\
\left\{\frac{c_{3}}{x}+\ldots\right\} x^{\theta_{\infty}-\theta_{1}+1} & -c_{1}+\ldots
\end{array}\right) .
\end{gathered}
$$

The role of $\theta_{\infty}-\theta_{1}+1$ and $\theta_{1}-\theta_{\infty}+1$ is just exchanged. By continuity, the matrices $A_{0}, A_{x}, A_{1}$ computed above hold for any value of $\theta_{1}-\theta_{\infty} \notin \mathbf{Z}, \theta_{\infty} \neq 1$.

The matching procedure can be repeated in the same way in case $\sigma=-\left(\theta_{\infty}-\theta_{1}\right)$ and in the case $\sigma= \pm\left(\theta_{\infty}+\theta_{1}\right)$, which yields (21). For this last cases the results are obtained just by the substitution $\theta_{1} \mapsto-\theta_{1}$.

\subsection{Monodromy Data}

We compute the monodromy data for the case $\sigma=\theta_{1}-\theta_{\infty}$, all the other cases being analogous. In this case, the matching has been realized by:

$$
\begin{gathered}
\Psi_{O U T}(\lambda, x):=\left(\begin{array}{cc}
\lambda^{\frac{\theta_{1}-\theta_{\infty}}{2}}(1-\lambda)^{-\frac{\theta_{1}}{2}} & -\frac{r_{1}(x)}{\theta_{1}} \lambda^{\frac{\theta_{\infty}-\theta_{1}}{2}-1}(1-\lambda)^{\frac{\theta 1}{2}} \\
0 & \lambda^{\frac{\theta_{\infty}-\theta_{1}}{2}}(1-\lambda)^{\frac{\theta_{1}}{2}}
\end{array}\right) . \\
\Psi_{I N}(\lambda, x)=K_{0}(x) \Psi_{0}\left(\frac{\lambda}{x}\right), \quad \Psi_{0}(\mu)=\left[I+O\left(\frac{1}{\mu}\right)\right] \mu^{\frac{\theta_{1}-\theta_{\infty}}{2} \sigma_{3}}, \quad \mu \rightarrow \infty .
\end{gathered}
$$

Let $\Psi(\lambda)$ denote the solution of the system (4) of (PVI), such that: $\Psi(\lambda)=\left[I+O\left(\lambda^{-1}\right)\right] \lambda^{-\frac{\theta_{\infty}}{2} \sigma_{3}}$, $\lambda \rightarrow \infty, \theta_{\infty} \notin \mathbf{Z}$.

\section{MATCHING $\Psi \leftrightarrow \Psi_{O U T}$.}

With the choice $1-\lambda=e^{-i \pi}(\lambda-1)(1-\lambda>0$ for $0<\lambda<1)$ we have:

$$
\Psi_{\text {OUT }}=\left[I+O\left(\frac{1}{\lambda}\right)\right] \lambda^{-\frac{\theta_{\infty}}{2} \sigma_{3}} e^{i \frac{\pi}{2} \theta_{1} \sigma_{3}}, \quad \lambda \rightarrow \infty
$$

Therefore, the correct choice for $\Psi_{O U T}$, which matches with $\Psi$ is:

$$
\Psi_{\text {OUT }}^{\text {Match }}:=\Psi_{\text {OUT }} C_{\text {OUT }}, \quad C_{\text {OUT }}:=e^{-i \frac{\pi}{2} \theta_{1} \sigma_{3}} .
$$

As a consequence we obtain:

i) the monodromy of $\Psi$ at $\lambda=1, \infty$, which is equal to the monodromy of $\Psi_{O U T}^{M a t c h}$ :

$$
M_{1}=e^{-i \pi \theta_{1} \sigma_{3}}, \quad M_{\infty}=e^{-i \pi \theta_{\infty} \sigma_{3}} ;
$$


ii) the correct choice for $\Psi_{I N}^{M a t c h}:=K_{0}(x) \Psi_{0} C_{O U T}$.

\section{MATCHING $\Psi \leftrightarrow \Psi_{I N}^{M a t c h}$}

This is realized by construction. A consequence, we can compute the monodromy of $\Psi$ at $\lambda=0, x$. In order to do this, we need the local behavior of $\Psi_{I N}^{M a t c h}(\lambda, x)$ at $\lambda=0, x$. We start with $\Psi_{0}$, recalling that:

$$
\Psi_{0}(\mu)=\mu^{\frac{\theta_{0}}{2}}(\mu-1)^{\frac{\theta_{x}}{2}} \Phi_{0}(\mu), \quad \Phi_{0}(\mu)=\left(\begin{array}{cc}
\varphi_{1}(\mu) & \varphi_{2}(\mu) \\
\xi_{1}(\mu) & \xi_{2}(\mu)
\end{array}\right)
$$

Here, $\varphi_{1}, \varphi_{2}$ are two independent solutions of a Gauss hyper-geometric equation (see (108) in Appendix 1):

$$
\mu(1-\mu) \frac{d^{2} \varphi}{d \mu^{2}}+(1+c-(a+[b+1]+1) \mu) \frac{d \varphi}{d \mu}-a(b+1) \varphi=0,
$$

where $a:=\frac{\theta_{0}}{2}+\frac{\theta_{x}}{2}+\frac{\theta_{\infty}}{2}-\frac{\theta_{1}}{2}, b+1:=\frac{\theta_{0}}{2}+\frac{\theta_{x}}{2}+\frac{\theta_{1}}{2}-\frac{\theta_{\infty}}{2}+1, c+1:=\theta_{0}+1$. The functions $\xi_{1}$ and $\xi_{2}$ are obtained from $\varphi_{1}$ and $\varphi_{2}$ by:

$$
\xi=\frac{1}{r}\left[\mu(1-\mu) \frac{d \varphi}{d \mu}-a\left(\mu+\frac{b-c}{a-b}\right) \varphi\right]
$$

In order to have generic solutions (i.e. non-logarithmic solutions) of the hyper-geometric equation, we must require:

$$
\theta_{1}-\theta_{\infty}, \theta_{0}, \theta_{x} \notin \mathbf{Z}
$$

Then, we have the following sets of independent solutions at $\mu=0,1, \infty$ respectively (we denote by $F$ the Gauss hyper-geometric function):

$$
\begin{aligned}
& \left\{\begin{array}{c}
\varphi_{1}^{(0)}=F\left(\frac{\theta_{0}}{2}+\frac{\theta_{x}}{2}+\frac{\theta_{\infty}}{2}-\frac{\theta_{1}}{2}, \frac{\theta_{0}}{2}+\frac{\theta_{x}}{2}+\frac{\theta_{1}}{2}-\frac{\theta_{\infty}}{2}+1,1+\theta_{0} ; \mu\right), \\
\varphi_{2}^{(0)}=\mu^{-\theta_{0}} F\left(\frac{\theta_{x}}{2}-\frac{\theta_{0}}{2}+\frac{\theta_{\infty}}{2}-\frac{\theta_{1}}{2}, \frac{\theta_{x}}{2}-\frac{\theta_{0}}{2}+\frac{\theta_{1}}{2}-\frac{\theta_{\infty}}{2}+1,1-\theta_{0} ; \mu\right) .
\end{array}\right. \\
& \left\{\begin{array}{c}
\varphi_{1}^{(1)}=F\left(\frac{\theta_{0}}{2}+\frac{\theta_{x}}{2}+\frac{\theta_{\infty}}{2}-\frac{\theta_{1}}{2}, \frac{\theta_{0}}{2}+\frac{\theta_{x}}{2}+\frac{\theta_{1}}{2}-\frac{\theta_{\infty}}{2}+1,1+\theta_{x} ; 1-\mu\right), \\
\varphi_{2}^{(1)}=(1-\mu)^{-\theta_{x}} F\left(\frac{\theta_{0}}{2}-\frac{\theta_{x}}{2}+\frac{\theta_{\infty}}{2}-\frac{\theta_{1}}{2}, \frac{\theta_{0}}{2}-\frac{\theta_{x}}{2}+\frac{\theta_{1}}{2}-\frac{\theta_{\infty}}{2}+1,1-\theta_{x} ; 1-\mu\right) .
\end{array}\right. \\
& \left\{\begin{array}{c}
\varphi_{1}^{(\infty)}=\mu^{-\frac{\theta_{0}}{2}-\frac{\theta_{x}}{2}-\frac{\theta_{\infty}}{2}+\frac{\theta_{1}}{2}} F\left(\frac{\theta_{0}}{2}+\frac{\theta_{x}}{2}+\frac{\theta_{\infty}}{2}-\frac{\theta_{1}}{2}, \frac{\theta_{x}}{2}-\frac{\theta_{0}}{2}+\frac{\theta_{\infty}}{2}-\frac{\theta_{1}}{2}, \theta_{\infty}-\theta_{1} ; \frac{1}{\mu}\right), \\
\varphi_{2}^{(\infty)}=\mu^{-\frac{\theta_{0}}{2}-\frac{\theta_{x}}{2}-\frac{\theta_{1}}{2}+\frac{\theta_{\infty}}{2}-1} F\left(\frac{\theta_{0}}{2}+\frac{\theta_{x}}{2}+\frac{\theta_{1}}{2}-\frac{\theta_{\infty}}{2}+1, \frac{\theta_{x}}{2}-\frac{\theta_{0}}{2}+\frac{\theta_{1}}{2}-\frac{\theta_{\infty}}{2}+1,2+\theta_{1}-\theta_{\infty} ; \frac{1}{\mu}\right) .
\end{array}\right.
\end{aligned}
$$

The connection formulae can be found in any book on special functions:

$$
\begin{gathered}
{\left[\varphi_{1}^{(0)}, \varphi_{2}^{(0)}\right]=\left[\varphi_{1}^{(1)}, \varphi_{2}^{(1)}\right] C_{01}, \quad-\pi<\arg (1-\mu)<\pi .} \\
{\left[\varphi_{1}^{(0)}, \varphi_{2}^{(0)}\right]=\left[\varphi_{1}^{(\infty)}, \varphi_{2}^{(\infty)}\right] C_{0 \infty}, \quad 0<\arg \mu<2 \pi .}
\end{gathered}
$$

Here the connection matrices $C_{01}, C_{0 \infty}$ are (34) and (33) respectively.

From the Taylor expansion of the hyper-geometric functions in $\varphi_{i}^{(\infty)}$ and (96) we compute:

$$
\begin{gathered}
\mu^{\frac{\theta_{0}}{2}}(\mu-1)^{\frac{\theta_{x}}{2}} \varphi_{1}^{(\infty)}=\left[1+O\left(\frac{1}{\mu}\right)\right] \mu^{\frac{\theta_{1}-\theta_{\infty}}{2}}, \quad \mu^{\frac{\theta_{0}}{2}}(\mu-1)^{\frac{\theta_{x}}{2}} \varphi_{2}^{(\infty)}=\frac{1}{\mu}\left[1+O\left(\frac{1}{\mu}\right)\right] \mu^{\frac{\theta_{\infty}-\theta_{1}}{2}}, \\
\mu^{\frac{\theta_{0}}{2}}(\mu-1)^{\frac{\theta_{x}}{2}} \xi_{1}^{(\infty)}=\left[1+O\left(\frac{1}{\mu}\right)\right] \mu^{\frac{\theta_{1}-\theta_{\infty}}{2}}, \quad \mu^{\frac{\theta_{0}}{2}}(\mu-1)^{\frac{\theta_{x}}{2}} \xi_{2}^{(\infty)}=\frac{\theta_{1}-\theta_{\infty}+1}{r_{0}}\left[1+O\left(\frac{1}{\mu}\right)\right] \mu^{\frac{\theta_{\infty}-\theta_{1}}{2}},
\end{gathered}
$$


It follows that the matrix $\Psi_{0}(\mu)=\mu^{\frac{\theta_{0}}{2}}(\mu-1)^{\frac{\theta_{x}}{2}} \Phi_{0}(\mu)$ with the prescribed behavior $\left[I+O\left(\frac{1}{\mu}\right)\right] \mu^{\frac{\theta_{1}-\theta_{\infty}}{2} \sigma_{3}}$ at $\mu=\infty$ is:

$$
\Psi_{0}(\mu)=\mu^{\frac{\theta_{0}}{2}}(\mu-1)^{\frac{\theta_{x}}{2}}\left(\begin{array}{ll}
\varphi_{1}^{(\infty)} & \varphi_{2}^{(\infty)} \\
\xi_{1}^{(\infty)} & \xi_{2}^{(\infty)}
\end{array}\right)\left(\begin{array}{cc}
1 & 0 \\
0 & \frac{r}{\theta_{1}-\theta_{\infty}+1}
\end{array}\right)
$$

Let:

$$
C:=\left(\begin{array}{cc}
1 & 0 \\
0 & \frac{r}{\theta_{1}-\theta_{\infty}+1}
\end{array}\right) C_{O U T} \equiv\left(\begin{array}{cc}
1 & 0 \\
0 & \frac{r}{\theta_{1}-\theta_{\infty}+1}
\end{array}\right) e^{-i \frac{\pi}{2} \theta_{1} \sigma_{3}} .
$$

We conclude that:

$$
\begin{aligned}
\Psi_{I N}^{M a t c h}(\lambda, x) & =K_{0}(x)\left(\frac{\lambda}{x}\right)^{\frac{\theta_{0}}{2}}\left(\frac{\lambda}{x}-1\right)^{\frac{\theta_{x}}{2}}\left(\begin{array}{ll}
\varphi_{1}^{(\infty)}\left(\frac{\lambda}{x}\right) & \varphi_{2}^{(\infty)}\left(\frac{\lambda}{x}\right) \\
\xi_{1}^{(\infty)}\left(\frac{\lambda}{x}\right) & \xi_{2}^{(\infty)}\left(\frac{\lambda}{x}\right)
\end{array}\right) C, \\
& =K_{0}(x)\left(\frac{\lambda}{x}\right)^{\frac{\theta_{0}}{2}}\left(\frac{\lambda}{x}-1\right)^{\frac{\theta_{x}}{2}}\left(\begin{array}{cc}
\varphi_{1}^{(0)}\left(\frac{\lambda}{x}\right) & \varphi_{2}^{(0)}\left(\frac{\lambda}{x}\right) \\
\xi_{1}^{(0)}\left(\frac{\lambda}{x}\right) & \xi_{2}^{(0)}\left(\frac{\lambda}{x}\right)
\end{array}\right) C_{0 \infty}^{-1} C, \\
& =K_{0}(x)\left(\frac{\lambda}{x}\right)^{\frac{\theta_{0}}{2}}\left(\frac{\lambda}{x}-1\right)^{\frac{\theta_{x}}{2}}\left(\begin{array}{cc}
\varphi_{1}^{(1)}\left(\frac{\lambda}{x}\right) & \varphi_{2}^{(1)}\left(\frac{\lambda}{x}\right) \\
\xi_{1}^{(1)}\left(\frac{\lambda}{x}\right) & \xi_{2}^{(1)}\left(\frac{\lambda}{x}\right)
\end{array}\right) C_{01} C_{0 \infty}^{-1} C .
\end{aligned}
$$

The behaviors of the above matrix at $\lambda=x, 0$ is easily computed from:

$$
\begin{gathered}
\mu^{\frac{\theta_{0}}{2}}(\mu-1)^{\frac{\theta_{x}}{2}} \varphi_{1}^{(1)}= \\
=\mu^{\frac{\theta_{0}}{2}}(\mu-1)^{\frac{\theta_{x}}{2}} F(\ldots ; 1-\mu)=x^{-\frac{\theta_{0}}{2}-\frac{\theta_{x}}{2}} F\left(\ldots ; \frac{x-\lambda}{x}\right) \lambda^{\frac{\theta_{0}}{2}}(\lambda-x)^{\frac{\theta_{x}}{2}} \\
\mu^{\frac{\theta_{0}}{2}}(\mu-1)^{\frac{\theta_{x}}{2}} \varphi_{2}^{(1)}= \\
=\mu^{\frac{\theta_{0}}{2}}(\mu-1)^{\frac{\theta_{x}}{2}}(1-\mu)^{-\frac{\theta_{x}}{2}}=(-1)^{\theta_{x}} x^{-\frac{\theta_{0}}{2}+\frac{\theta_{x}}{2}} F\left(\ldots ; \frac{x-\lambda}{x}\right) \lambda^{\frac{\theta_{0}}{2}}(\lambda-x)^{-\frac{\theta_{x}}{2}}
\end{gathered}
$$

and:

$$
\begin{aligned}
& \mu^{\frac{\theta_{0}}{2}}(\mu-1)^{\frac{\theta_{x}}{2}} \varphi_{1}^{(0)}= \\
& \quad=\mu^{\frac{\theta_{0}}{2}}(\mu-1)^{\frac{\theta_{x}}{2}} F(\ldots ; \mu)=x^{-\frac{\theta_{0}}{2}-\frac{\theta_{x}}{2}} F\left(\ldots ; \frac{\lambda}{x}\right)(\lambda-x)^{\frac{\theta_{x}}{2}} \lambda^{\frac{\theta_{0}}{2}}, \\
& \mu^{\frac{\theta_{0}}{2}}(\mu-1)^{\frac{\theta_{x}}{2}} \varphi_{2}^{(0)}= \\
& =\mu^{-\frac{\theta_{0}}{2}}(\mu-1)^{\frac{\theta_{x}}{2}} F(\ldots ; \mu)=x^{\frac{\theta_{0}}{2}-\frac{\theta_{x}}{2}} F\left(\ldots ; \frac{\lambda}{x}\right)(\lambda-x)^{\frac{\theta_{x}}{2}} \lambda^{-\frac{\theta_{0}}{2}} .
\end{aligned}
$$

From these we compute:

$$
\begin{gathered}
K_{0}(x) \mu^{\frac{\theta_{0}}{2}}(\mu-1)^{\frac{\theta_{x}}{2}}\left(\begin{array}{ll}
\varphi_{1}^{(0)} & \varphi_{2}^{(0)} \\
\xi_{1}^{(0)} & \xi_{2}^{(0)}
\end{array}\right)=\psi_{0}^{I N}(x)(I+O(\lambda)) \lambda^{\frac{\theta_{0}}{2} \sigma_{3}}, \quad \lambda \rightarrow 0 \\
K_{0}(x) \mu^{\frac{\theta_{0}}{2}}(\mu-1)^{\frac{\theta_{x}}{2}}\left(\begin{array}{ll}
\varphi_{1}^{(1)} & \varphi_{2}^{(1)} \\
\xi_{1}^{(1)} & \xi_{2}^{(1)}
\end{array}\right)=\psi_{x}^{I N}(x)(I+O(\lambda-x))(\lambda-x)^{\frac{\theta_{x}}{2} \sigma_{3}}, \quad \lambda \rightarrow x .
\end{gathered}
$$

Here, we don't need to explicitly give the invertible matrices $\psi_{0}^{I N}(x)$ and $\psi_{x}^{I N}(x)$. From the above procedure, we find:

$$
M_{0}=C^{-1}\left(C_{0 \infty} \exp \left\{i \pi \theta_{0} \sigma_{3}\right\} C_{0 \infty}^{-1}\right) C, \quad M_{x}=C^{-1}\left[C_{0 \infty}\left(C_{01}^{-1} \exp \left\{i \pi \theta_{x} \sigma_{3}\right\} C_{01}\right) C_{0 \infty}^{-1}\right] C .
$$

We finally observe that $M_{1}$ and $M_{\infty}$ are diagonal, so they are invariant for the conjugation $M \mapsto$ $C M C^{-1}$. With this in mind, we get the result of theorem 3, point a).

We stress that $\hat{A}_{0}+\hat{A}_{x}$ and $\hat{A}_{1}$ are upper (or lower) triangular matrices, and the group generated by $M_{x} M_{0}$ and $M_{1}$ is reducible. 


\section{$7 \quad$ Case $\theta_{\infty}=1, \theta_{1}=0, \sigma= \pm 1$. Solution (20).}

In section 6 we imposed that $\theta_{\infty} \neq 1$ and $\theta_{\infty}-\theta_{1} \notin \mathbf{Z}$. Here we consider the case $\theta_{\infty}=1, \theta_{\infty}-\theta_{1}=1$. We have:

$$
\hat{A_{0}}+\hat{A_{x}}=\left(\begin{array}{cc}
-\frac{1}{2} & r_{1} \\
0 & \frac{1}{2}
\end{array}\right), \quad \hat{A_{1}}=\left(\begin{array}{cc}
0 & -r_{1} \\
0 & 0
\end{array}\right) .
$$

Also the transpose matrices are possible. For $|\lambda| \leq|x|^{\delta_{I N}}$, we use the reduction (46) (namely, (14)). The matrices $\hat{\hat{A}_{0}}, \hat{\hat{A_{x}}}$ are given by (47) and (48) with the substitution $\sigma=1$ or -1 . For definiteness, let us take $\sigma=1$ in the following.

For $|\lambda| \geq|x|^{\delta_{\text {OUT }}}$, we approximate (4) with: $\frac{d \Psi_{\text {OUT }}}{d \lambda}=\left[\frac{x A_{x}}{\lambda^{2}}+\frac{A_{0}+A_{x}}{\lambda}+\frac{A_{1}}{\lambda-1}\right] \Psi_{\text {OUT }}$. For definiteness, let us consider the case when $\hat{A}_{1}$ and $\hat{A}_{0}+\hat{A_{x}}$ are upper triangular. Again, we make the hypothesis that the leading terms in $x A_{x}$ define an upper triangular matrix:

$$
x A_{x}=:\left(\begin{array}{ll}
0 & \rho \\
0 & 0
\end{array}\right)+\text { higher orders. }
$$

Therefore, we will study:

$$
\frac{d \Psi_{\text {OUT }}}{d \lambda}=\left[\frac{1}{\lambda^{2}}\left(\begin{array}{cc}
0 & \rho \\
0 & 0
\end{array}\right)+\frac{1}{\lambda}\left(\begin{array}{cc}
-\frac{1}{2} & r_{1} \\
0 & \frac{1}{2}
\end{array}\right)+\frac{1}{\lambda-1}\left(\begin{array}{cc}
0 & -r_{1} \\
0 & 0
\end{array}\right)\right] \Psi_{O U T}
$$

This is a reducible system. To solve it, we write $\Psi_{\text {OUT }}$ in vector notation $\Psi_{\text {OUT }}=\left(\begin{array}{l}\psi_{1} \\ \psi_{2}\end{array}\right)$. The system becomes:

$$
\frac{d \psi_{1}}{d \lambda}=-\frac{1}{2 \lambda} \psi_{1}+\left(\frac{\rho}{\lambda^{2}}+\frac{r_{1}}{\lambda}-\frac{r_{1}}{\lambda-1}\right) \psi_{2}, \quad \frac{d \psi_{2}}{d \lambda}=\frac{1}{2 \lambda} \psi_{2} .
$$

The solution obtained by variation of parameters is:

$$
\psi_{1}=C_{1} \lambda^{-\frac{1}{2}}+C_{2} \lambda^{-\frac{1}{2}}\left(\rho \ln \lambda-r_{1} \ln (\lambda-1)\right), \quad \psi_{2}=C_{2} \lambda^{\frac{1}{2}}, \quad C_{1}, C_{2} \in \mathbf{C} .
$$

We can choose the following fundamental matrix:

$$
\Psi_{\text {OUT }}=\left(\begin{array}{cc}
\lambda^{-\frac{1}{2}} & \lambda^{-\frac{1}{2}}\left(\rho \ln \lambda-r_{1} \ln (\lambda-1)\right) \\
0 & \lambda^{\frac{1}{2}}
\end{array}\right)=\left(\begin{array}{cc}
\lambda^{-\frac{1}{2}} & 0 \\
0 & \lambda^{\frac{1}{2}}
\end{array}\right)\left(\begin{array}{cc}
1 & \rho \ln \lambda \\
0 & 1
\end{array}\right)\left(\begin{array}{cc}
1 & -r_{1} \ln (\lambda-1) \\
0 & 1
\end{array}\right) .
$$

Its monodromy relative to the loops $\lambda \mapsto \lambda e^{2 \pi i}$ and $(\lambda-1) \mapsto(\lambda-1) e^{2 \pi i}$ is respectively:

$$
\Psi_{\text {OUT }} \mapsto \Psi_{\text {OUT }}\left(\begin{array}{cc}
-1 & -2 \pi i \rho \\
0 & -1
\end{array}\right) . \quad \Psi_{\text {OUT }} \mapsto \Psi_{\text {OUT }}\left(\begin{array}{cc}
1 & -2 \pi i r_{1} \\
0 & 1
\end{array}\right) .
$$

Therefore, $\rho$ and $r_{1}$ must be independent of (the monodromy preserving deformation) $x$. We observe that any fundamental matrix solution of the form:

$$
\Psi_{\text {OUT }}\left(\begin{array}{cc}
1 & f(x) \\
0 & 1
\end{array}\right)
$$

has the same monodromy of $\Psi_{O U T}$, for any arbitrary function of $x$. This fact will be used soon, with the choice $f(x)=-\rho \ln x$.

With the choice of the branch of $\ln (\lambda-1)=\ln (1-\lambda)+i \pi$ (i.e. $(\lambda-1)=e^{i \pi}(1-\lambda), 1-\lambda>0$ for $0<\lambda<1$ ), it is convenient to redefine $\Psi_{\text {OUT }}$ by:

$$
\begin{aligned}
\Psi_{\text {OUT }}:= & \left(\begin{array}{cc}
\lambda^{-\frac{1}{2}} & 0 \\
0 & \lambda^{\frac{1}{2}}
\end{array}\right)\left(\begin{array}{cc}
1 & \rho \ln \lambda \\
0 & 1
\end{array}\right)\left(\begin{array}{cc}
1 & -r_{1} \ln (\lambda-1) \\
0 & 1
\end{array}\right)\left(\begin{array}{cc}
1 & i \pi r_{1} \\
0 & 1
\end{array}\right)= \\
= & \left(\begin{array}{cc}
1 & -\frac{r_{1}}{\lambda} \ln (1-\lambda) \\
0 & 1
\end{array}\right)\left(\begin{array}{cc}
\lambda^{-\frac{1}{2}} & 0 \\
0 & \lambda^{\frac{1}{2}}
\end{array}\right)\left(\begin{array}{cc}
1 & \rho \ln \lambda \\
0 & 1
\end{array}\right) .
\end{aligned}
$$

Therefore:

$$
\Psi_{\text {OUT }}=\left[\left(\begin{array}{cc}
1 & r_{1} \\
0 & 1
\end{array}\right)+\left(\begin{array}{cc}
0 & i \pi r_{1} \\
0 & 0
\end{array}\right) \sum_{n=1}^{\infty} \frac{\lambda^{n}}{n+1}\right]\left(\begin{array}{cc}
\lambda^{-\frac{1}{2}} & 0 \\
0 & \lambda^{\frac{1}{2}}
\end{array}\right)\left(\begin{array}{cc}
1 & \rho \ln \lambda \\
0 & 1
\end{array}\right), \quad \lambda \rightarrow 0
$$




\subsection{Matching}

The solution $\Psi_{0}\left(\frac{\lambda}{x}\right)$ has been introduced in section 4.7 .

$$
\Psi_{0}\left(\frac{\lambda}{x}\right)=\left[I+O\left(\frac{x}{\lambda}\right)\right]\left(\begin{array}{cc}
\left(\frac{\lambda}{x}\right)^{\frac{1}{2}} & 0 \\
0 & \left(\frac{\lambda}{x}\right)^{-\frac{1}{2}}
\end{array}\right)\left(\begin{array}{cc}
1 & 0 \\
R \ln \left(\frac{\lambda}{x}\right) & 1
\end{array}\right), \quad R:=\left(\hat{\hat{A_{x}}}\right)_{21} .
$$

Some adjustments are necessary. Let us consider the permutation matrix $P:=\left(\begin{array}{ll}0 & 1 \\ 1 & 0\end{array}\right)$ and redefine:

$$
\Psi_{0} \mapsto \Psi_{0} P\left(\begin{array}{cc}
1 & 0 \\
0 & R^{-1}
\end{array}\right), \quad \Psi_{\text {OUT }} \mapsto \Psi_{\text {OUT }}\left(\begin{array}{cc}
1 & -\rho \ln x \\
0 & 1
\end{array}\right)\left(\begin{array}{cc}
1 & 0 \\
0 & \rho^{-1}
\end{array}\right) .
$$

As we have already observed, this re-definition does not affect the monodromy of $\Psi_{\text {OUT }}$, which is independent of $x$. The matching relation $\Psi_{O U T}(\lambda) \sim K_{0}(x) \Psi_{0}(\lambda / x)$ becomes:

$$
\begin{aligned}
\left(\begin{array}{cc}
1 & r_{1} \\
0 & 1
\end{array}\right)\left(\begin{array}{cc}
1 & 0 \\
0 & \frac{1}{\rho}
\end{array}\right) & \left(\begin{array}{cc}
\lambda^{-\frac{1}{2}} & 0 \\
0 & \lambda^{\frac{1}{2}}
\end{array}\right)\left(\begin{array}{cc}
1 & \ln \frac{\lambda}{x} \\
0 & 1
\end{array}\right) \sim \\
& \sim K_{0}(x) P\left(\begin{array}{cc}
1 & 0 \\
0 & \frac{1}{R}
\end{array}\right)\left(\begin{array}{cc}
x^{\frac{1}{2}} & 0 \\
0 & x^{-\frac{1}{2}}
\end{array}\right)\left(\begin{array}{cc}
\lambda^{-\frac{1}{2}} & 0 \\
0 & \lambda^{\frac{1}{2}}
\end{array}\right)\left(\begin{array}{cc}
1 & \ln \frac{\lambda}{x} \\
0 & 1
\end{array}\right) .
\end{aligned}
$$

The matching is thus realized, with the choice:

$$
K_{0}(x) \sim\left(\begin{array}{cc}
1 & \frac{r_{1}}{\rho} \\
0 & \frac{1}{\rho}
\end{array}\right)\left(\begin{array}{cc}
x^{-\frac{1}{2}} & 0 \\
0 & R x^{\frac{1}{2}}
\end{array}\right) P \equiv\left(\begin{array}{cc}
\frac{r_{1}}{\rho} & 1 \\
\frac{1}{\rho} & 0
\end{array}\right)\left(\begin{array}{cc}
R x^{\frac{1}{2}} & 0 \\
0 & x^{-\frac{1}{2}}
\end{array}\right) .
$$

It follows that:

$$
\begin{gathered}
\hat{A}_{0}=\left(\begin{array}{cc}
\frac{\theta_{x}^{2}-\theta_{0}^{2}-1}{4}+\frac{r_{1}}{\rho} \frac{\left[1-\left(\theta_{0}-\theta_{x}\right)^{2}\right]\left[1-\left(\theta_{0}+\theta_{x}\right)^{2}\right]}{16} x & -\frac{\rho}{x}+r_{1} \frac{\theta_{0}^{2}-\theta_{x}^{2}+1}{2}-\frac{r_{1}^{2}}{\rho} \frac{\left.\left[1-\left(\theta_{0}-\theta_{x}\right)^{2}\right)\right]\left[1-\left(\theta_{0}+\theta_{x}\right)^{2}\right]}{16} x \\
\frac{1}{\rho} \frac{\left.\left[1-\left(\theta_{0}-\theta_{x}\right)^{2}\right)\right]\left[1-\left(\theta_{0}+\theta_{x}\right)^{2}\right]}{16} x & -\frac{\theta_{x}^{2}-\theta_{0}^{2}-1}{4}-\frac{r_{1}}{\rho} \frac{\left[1-\left(\theta_{0}-\theta_{x}\right)^{2}\right]\left[1-\left(\theta_{0}+\theta_{x}\right)^{2}\right]}{16} x
\end{array}\right), \\
\hat{A}_{x}=\left(\begin{array}{cc}
\frac{\theta_{0}^{2}-\theta_{x}^{2}-1}{4}-\frac{r_{1}}{\rho} \frac{\left[1-\left(\theta_{0}-\theta_{x}\right)^{2}\right]\left[1-\left(\theta_{0}+\theta_{x}\right)^{2}\right]}{16} x & \frac{\rho}{x}+r_{1} \frac{\theta_{x}^{2}-\theta_{0}^{2}+1}{2}+\frac{r_{1}^{2}}{\rho} \frac{\left[1-\left(\theta_{0}-\theta_{x}\right)^{2}\right]\left[1-\left(\theta_{0}+\theta_{x}\right)^{2}\right]}{16} x \\
-\frac{1}{\rho} \frac{\left[1-\left(\theta_{0}-\theta_{x}\right)^{2}\right]\left[1-\left(\theta_{0}+\theta_{x}\right)^{2}\right]}{16} x & -\frac{\theta_{0}^{2}-\theta_{x}^{2}-1}{4}+\frac{r_{1}}{\rho} \frac{\left[1-\left(\theta_{0}-\theta_{x}\right)^{2}\right]\left[1-\left(\theta_{0}+\theta_{x}\right)^{2}\right]}{16} x
\end{array}\right) .
\end{gathered}
$$

The leading term of each matrix element certainly contains no error. Note that $r$ has simplified. Actually, the constant $\rho$ plays the role of $r$.

We will not repeat again the discussion for the higher order terms. The matrix elements are Taylor expansions, corresponding to a Taylor expanded $y(x)$, the convergence of which is proved by a Briot-Bouquet argument. The first two leading terms of $A_{0}$ and $A_{x}$ above are actually correct. In particular, we need:

$$
\left(A_{0}\right)_{12}=-\frac{\rho}{x}+r_{1} \frac{\theta_{0}^{2}-\theta_{x}^{2}+1}{2}+O(x) .
$$

The leading terms of $A_{1}$ are:

$$
A_{1}=\left(\begin{array}{cc}
\frac{r_{1}}{\rho} \frac{\left[1-\left(\theta_{0}-\theta_{x}\right)^{2}\right]\left[1-\left(\theta_{0}+\theta_{x}\right)^{2}\right]}{32} x^{2}+O\left(x^{3}\right) & -r_{1}+r_{1} \frac{\theta_{0}^{2}-\theta_{x}^{2}-1}{2} x+O\left(x^{2}\right) \\
\frac{r_{1}}{\rho^{2}} \frac{\left[1-\left(\theta_{0}-\theta_{x}\right)^{2}\right]^{2}\left[1-\left(\theta_{0}+\theta_{x}\right)^{2}\right]^{2}}{1024} x^{4}+O\left(x^{5}\right) & -\frac{r_{1}}{\rho} \frac{\left[1-\left(\theta_{0}-\theta_{x}\right)^{2}\right]\left[1-\left(\theta_{0}+\theta_{x}\right)^{2}\right]}{32} x^{2}+O\left(x^{3}\right)
\end{array}\right) .
$$

The above truncations of $\left(A_{1}\right)_{12}$ and $\left(A_{0}\right)_{12}$ corresponding to the first two terms of the Taylor expansion of the solution (20), through (6):

$$
y(x)=a+\frac{1-a}{2}\left(1+\theta_{0}^{2}-\theta_{x}^{2}\right) x+O\left(x^{2}\right), \quad \text { where } a:=\left(1-\frac{r_{1}}{\rho}\right)^{-1} .
$$

Observe that $a$ depends on the monodromy datum $\frac{r_{1}}{\rho}$. 


\subsection{Monodromy}

The matching $\Psi_{I N} \leftrightarrow \Psi_{\text {OUT }}$ has been realized by:

$$
\Psi_{\text {OUT }}=\left(\begin{array}{cc}
\lambda^{-\frac{1}{2}} & 0 \\
0 & \lambda^{\frac{1}{2}}
\end{array}\right)\left(\begin{array}{cc}
1 & \rho \ln \lambda \\
0 & 1
\end{array}\right)\left(\begin{array}{cc}
1 & -r_{1} \ln (\lambda-1) \\
0 & 1
\end{array}\right)\left(\begin{array}{cc}
1 & -\ln x+i \pi \frac{r_{1}}{\rho} \\
0 & \frac{1}{\rho}
\end{array}\right)
$$

and:

$$
\begin{gathered}
\Psi_{I N}(\mu)=K_{0}(x) \Psi_{0}(\mu)\left(\begin{array}{cc}
0 & R^{-1} \\
1 & 0
\end{array}\right), \quad R=\frac{\left[\left(\theta_{0}+\theta_{x}\right)^{2}-1\right]\left[\left(\theta_{0}-\theta_{x}\right)^{2}-1\right]}{16 r} . \\
\Psi_{0}(\mu)=\left[I+O\left(\frac{1}{\mu}\right)\right]\left(\begin{array}{cc}
\mu^{\frac{1}{2}} & 0 \\
0 & \mu^{-\frac{1}{2}}
\end{array}\right)\left(\begin{array}{cc}
1 & 0 \\
R \ln \mu & 1
\end{array}\right), \quad \mu \rightarrow \infty .
\end{gathered}
$$

Namely:

$$
\begin{aligned}
\Psi_{I N} \sim K_{0}(x) P\left(\begin{array}{cc}
x^{\frac{1}{2}} & 0 \\
0 & R^{-1} x^{-\frac{1}{2}}
\end{array}\right) & \left(\begin{array}{cc}
\lambda^{-\frac{1}{2}} & 0 \\
0 & \lambda^{\frac{1}{2}}
\end{array}\right)\left(\begin{array}{cc}
1 & \ln \frac{\lambda}{x} \\
0 & 1
\end{array}\right), \\
= & \left(\begin{array}{cc}
1 & \frac{r_{1}}{\rho} \\
0 & \frac{1}{\rho}
\end{array}\right)\left(\begin{array}{cc}
\lambda^{-\frac{1}{2}} & 0 \\
0 & \lambda^{\frac{1}{2}}
\end{array}\right)\left(\begin{array}{cc}
1 & \ln \frac{\lambda}{x} \\
0 & 1
\end{array}\right),
\end{aligned}
$$

for $\mu=\lambda / x \rightarrow \infty,|\lambda|<|x|^{\delta_{I N}}, x \rightarrow 0$.

\section{MATCHING $\Psi \leftrightarrow \Psi_{O U T}$.}

The correct choice of $\Psi_{\text {OUT }}$ must match with:

$$
\Psi=\left[I+O\left(\frac{1}{\lambda}\right)\right] \lambda^{-\frac{\theta_{\infty}}{2} \sigma_{3}} \lambda^{L}, \quad \lambda \rightarrow \infty ; \quad \theta_{\infty}=1 ;
$$

where: $L=\left(\begin{array}{cc}0 & \rho-r_{1} \\ 0 & 0\end{array}\right)$. This form of $L$ follows from the standard theory of Fuchsian systems, and from the expansion of the system (4) at $\nu:=\frac{1}{\lambda} \rightarrow 0$ :

$$
\frac{d \Psi}{d \nu}=\left[\frac{\sigma_{3}}{2} \frac{1}{\nu}-\left(A_{1}+x A_{x}\right)+O(\nu)\right] \Psi, \quad \nu \rightarrow \infty
$$

Thus $L_{12}=-\left.\left(A_{1}+x A_{x}\right)_{1,2}\right|_{x=0} \equiv r_{1}-\rho$ (this is computed from the expansions of $A_{1}$ and $A_{x}$ at $x=0$ obtained before). We expand $\Psi_{\text {OUT }}$ at $\lambda=\infty$. We easily get:

$$
\Psi_{\text {OUT }}=\left[I+O\left(\lambda^{-1}\right)\right] \lambda^{-\frac{1}{2} \sigma_{3}}\left(\begin{array}{cc}
1 & \left(\rho-r_{1}\right) \ln \lambda \\
0 & 1
\end{array}\right)\left(\begin{array}{cc}
1 & i \pi \frac{r_{1}}{\rho}-\ln x \\
0 & \frac{1}{\rho}
\end{array}\right) .
$$

Therefore, the correct choice is:

$$
\Psi_{\text {OUT }}^{\text {Match }}:=\Psi_{\text {OUT }}\left(\begin{array}{cc}
1 & i \pi \frac{r_{1}}{\rho}-\ln x \\
0 & \frac{1}{\rho}
\end{array}\right)^{-1}=\lambda^{-\frac{1}{2} \sigma_{3}}\left(\begin{array}{cc}
1 & \rho \ln \lambda \\
0 & 1
\end{array}\right)\left(\begin{array}{cc}
1 & -r_{1} \ln (\lambda-1) \\
0 & 1
\end{array}\right) .
$$

As a consequence, we obtain:

i) the monodromy of $\Psi$ at $\lambda=1, \infty$. This coincides with that of $\Psi_{O U T}^{\text {Match }}$, which is easily computed from the local behavior:

$$
\Psi_{\text {OUT }}^{\text {Match }}(\lambda)=[I+O(\lambda-1)]\left(\begin{array}{cc}
1 & -r_{1} \ln (\lambda-1) \\
0 & 1
\end{array}\right), \quad \lambda \rightarrow 1 .
$$

Thus, for $(\lambda-1) \mapsto(\lambda-1) \exp \{2 \pi i\}$ and $\lambda \mapsto \lambda \exp \{2 \pi i\}$ the monodromy is:

$$
M_{1}=\left(\begin{array}{cc}
1 & -2 \pi i r_{1} \\
0 & 1
\end{array}\right), \quad M_{\infty}=\left(\begin{array}{cc}
-1 & 2 \pi i\left(r_{1}-\rho\right) \\
0 & -1
\end{array}\right) .
$$


ii) the correct choice of $\Psi_{I N}$, which matches with $\Psi$. This is:

$$
\begin{aligned}
\Psi_{I N}^{\text {Match }}(\lambda, x)= & \Psi_{I N}(\lambda, x)\left(\begin{array}{cc}
1 & i \pi \frac{r_{1}}{\rho}-\ln x \\
0 & \frac{1}{\rho}
\end{array}\right)^{-1}=\Psi_{I N}(\lambda, x)\left(\begin{array}{cc}
1 & \rho \ln x-i \pi r_{1} \\
0 & \rho
\end{array}\right)= \\
& =K_{0}(x) \Psi_{0}(\mu) C_{I N}, \quad C_{I N}=\left(\begin{array}{cc}
0 & \rho / R \\
1 & \rho \ln x-i \pi r_{1}
\end{array}\right) .
\end{aligned}
$$

Important Remark: $C_{I N}$ depends on $x$. But the monodromy must be independent of $x$. For this reason, we will have to consider the substitution

$$
\Psi_{I N}^{\text {Match }} \mapsto \Psi_{I N}^{\text {Match }}\left(C_{I N}\right)^{-1}=K_{0}(x) \Psi_{0}(\mu) .
$$

This makes the monodromy at $\lambda=0, x$ independent of $x$. The corresponding transformation:

$$
\Psi_{\text {OUT }}^{\text {Match }} \mapsto \Psi_{\text {OUT }}^{\text {Match }} C_{I N}{ }^{-1},
$$

changes $M_{1}, M_{\infty}$, but does not introduce a dependence on $x$. Namely:

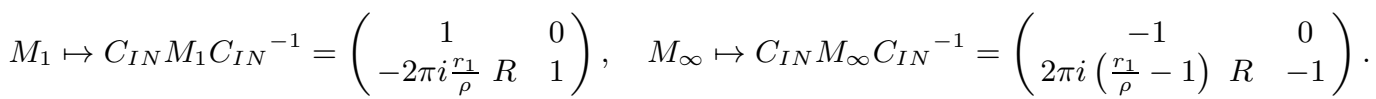

MATCHING $\Psi \leftrightarrow \Psi_{I N}$.

$\Psi$ and $\Psi_{I N}^{M a t c h}(\lambda, x)$ are matching by construction. The monodromy of $\Psi$ at $\lambda=0, x$ coincides with that of $\Psi_{I N}^{M a t c h}(\lambda, x)$. In order to compute it, we write:

$$
\Psi_{0}(\mu)=\mu^{\frac{\theta_{0}}{2}}(\mu-1)^{\frac{\theta_{x}}{2}} \Phi_{0}(\mu), \quad \Phi_{0}(\mu)=\left(\begin{array}{cc}
\varphi_{1} & \varphi_{2} \\
\xi_{1} & \xi_{2}
\end{array}\right)
$$

$\Phi_{0}$ satisfies (49) with $\sigma=1$. It is expressed in terms of two independent solutions $\varphi_{1}, \varphi_{2}$ of the Gauss hyper-geometric equation (see Appendix 1):

$$
\mu(1-\mu) \frac{d^{2} \varphi}{d \mu^{2}}+(1+c-(a+[b+1]+1) \mu) \frac{d \varphi}{d \mu}-a(b+1) \varphi=0,
$$

where,

$$
a=\frac{\theta_{0}}{2}+\frac{\theta_{x}}{2}-\frac{1}{2}, \quad b+1=\frac{\theta_{0}}{2}+\frac{\theta_{x}}{2}+\frac{3}{2}, \quad c+1=\theta_{0}+1 .
$$

From $\varphi_{1}$ and $\varphi_{2}$ we compute:

$$
\xi_{i}=\frac{1}{r}\left[\mu(1-\mu) \frac{d \varphi_{i}}{d \mu}-a\left(\mu+\frac{b-c}{a-b}\right) \varphi_{i}\right], \quad i=1,2 .
$$

We need a compete set of solutions at $\mu=0,1, \infty$. Since $a-b$ is an integer, we are in a logarithmic case. We briefly explain some preliminary facts. Let us consider a Gauss Hyper-geometric equation in standard form:

$$
\mu(1-\mu) \frac{d^{2} \varphi}{d \mu^{2}}+[\gamma-(\alpha+\beta+1) \mu] \frac{d \varphi}{d \mu}-\alpha \beta \varphi=0
$$

( $\alpha, \beta, \gamma$ here are not the coefficients of (PVI)! We are just using the same symbols only here). Logarithmic solutions at $\mu=0$ may occur only if $\gamma \in \mathbf{Z}$. Logarithmic solutions at $\mu=1$ may occur only if $\alpha+\beta-\gamma \in \mathbf{Z}$. Logarithmic solutions at $\mu=\infty$ may occur only if $\alpha-\beta \in \mathbf{Z}$. Several sub-case must be distinguished, and this is not the place to discuss them.

In our case $\alpha=a, \beta=b+1$ and $\gamma=c+1$. Therefore $\alpha-\beta=-2$. This case is logarithmic. Two independent solutions are:

$$
\varphi_{1}^{(\infty)}=\mu^{-\beta} g_{1}\left(\beta, \beta-\gamma+1,1+\beta-\alpha ; \frac{1}{\mu}\right)=[\text { in our case }] \mu^{-\alpha-2} g_{1}\left(\beta, \beta-\gamma+1,3 ; \frac{1}{\mu}\right),
$$




$$
\varphi_{2}^{(\infty)}=\mu^{-\beta} F\left(\beta, \beta-\gamma+1,1+\beta-\alpha ; \frac{1}{\mu}\right)=[\text { in our case }] \mu^{-\beta} F\left(\beta, \beta-\gamma+1,3 ; \frac{1}{\mu}\right) .
$$

Here $F$ is the Gauss hyper-geometric function and $g_{1}$ is a logarithmic solution introduced in Norlund's paper [23], page 7:

$$
\begin{gathered}
g_{1}(u, v, w ; z)=\sum_{n=1}^{w-1}(-1)^{n-1}(n-1) ! \frac{(u)_{-n}(v)_{-n}}{(w)_{-n}} \frac{1}{z^{n}}+F(u, v, w ; z) \ln (-z)+ \\
+\sum_{n=0}^{\infty} \frac{(u)_{n}(v)_{n}}{n !(w)_{n}}[\psi(1-u-n)+\psi(v+n)-\psi(w+n)-\psi(1+n)] z^{n}
\end{gathered}
$$

where

$$
\psi(x):=\frac{d}{d x} \ln \Gamma(x), \quad x \neq 0,-1,-2,-3, \ldots ; \quad|z|<1, \quad \ln (-z)<0 \text { for }-1<z<0 .
$$

Note that the first sum $\sum_{n=1}^{w-1}(-1)^{n-1}(n-1) ! \frac{(u)_{-n}(v)_{-n}}{(w)_{-n}} \frac{1}{z^{n}}$ is a polynomial in $1 / z$.

If $\theta_{0}, \theta_{x} \notin \mathbf{Z}$, we fall in the non-logarithmic cases at $\mu=0,1$. Thus:

$$
\begin{gathered}
\varphi_{1}^{(0)}=F\left(\frac{\theta_{0}}{2}+\frac{\theta_{x}}{2}-\frac{1}{2}, \frac{\theta_{0}}{2}+\frac{\theta_{x}}{2}+\frac{3}{2}, 1+\theta_{0} ; \mu\right), \\
\varphi_{2}^{(0)}=\mu^{-\theta_{0}} F\left(-\frac{\theta_{0}}{2}+\frac{\theta_{x}}{2}-\frac{1}{2},-\frac{\theta_{0}}{2}+\frac{\theta_{x}}{2}+\frac{3}{2}, 1-\theta_{0} ; \mu\right) . \\
\varphi_{1}^{(1)}=F\left(\frac{\theta_{0}}{2}+\frac{\theta_{x}}{2}-\frac{1}{2}, \frac{\theta_{0}}{2}+\frac{\theta_{x}}{2}+\frac{3}{2}, 1+\theta_{x} ; 1-\mu\right), \\
\varphi_{2}^{(1)}=(1-\mu)^{-\theta_{x}} F\left(\frac{\theta_{0}}{2}-\frac{\theta_{x}}{2}-\frac{1}{2}, \frac{\theta_{0}}{2}-\frac{\theta_{x}}{2}+\frac{3}{2}, 1-\theta_{x} ; 1-\mu\right) . \\
\varphi_{1}^{(\infty)}=\mu^{-\frac{\theta_{0}}{2}-\frac{\theta_{x}}{2}-\frac{3}{2}} g_{1}\left(\frac{\theta_{0}}{2}+\frac{\theta_{x}}{2}+\frac{3}{2},-\frac{\theta_{0}}{2}+\frac{\theta_{x}}{2}+\frac{3}{2}, 3 ; \frac{1}{\mu}\right), \\
\varphi_{2}^{(\infty)}=\mu^{-\frac{\theta_{0}}{2}-\frac{\theta_{x}}{2}-\frac{3}{2}} F\left(\frac{\theta_{0}}{2}+\frac{\theta_{x}}{2}+\frac{3}{2},-\frac{\theta_{0}}{2}+\frac{\theta_{x}}{2}+\frac{3}{2}, 3 ; \frac{1}{\mu}\right) .
\end{gathered}
$$

The connection matrix between $\left[\varphi_{1}^{(0)}, \varphi_{2}^{(0)}\right]$ and $\left[\varphi_{1}^{(1)}, \varphi_{2}^{(1)}\right]$ is a standard one:

$$
\left[\varphi_{1}^{(0)}, \varphi_{2}^{(0)}\right]=\left[\varphi_{1}^{(1)}, \varphi_{2}^{(1)}\right] C_{01}, \quad 0<\arg (1-\mu)<2 \pi
$$

where $C_{01}$ is $(36)$.

On the other hand, the connection matrix between $\left[\varphi_{1}^{(0)}, \varphi_{2}^{(0)}\right]$ and $\left[\varphi_{1}^{(\infty)}, \varphi_{2}^{(\infty)}\right]$ is computed in [23]. Our specific case falls in the case $\alpha-\beta=-p, p \geq 0$ integer. From [23], page 27, case 11, formulae (1) and (2), we deduce the connection formulae:

$$
\begin{gathered}
(-\mu)^{\alpha-\beta} F\left(\beta, \beta-\gamma+1,1+\beta-\alpha ; \frac{1}{\mu}\right)=\frac{\Gamma(\beta-\alpha+1) \Gamma(1-\gamma)}{\Gamma(1-\alpha) \Gamma(\beta-\gamma+1)}(-\mu)^{\alpha} F(\alpha, \beta, \gamma ; \mu)+ \\
+\frac{\Gamma(\beta-\alpha+1) \Gamma(\gamma-1)}{\Gamma(\gamma-\alpha) \Gamma(\beta)}(-\mu)^{\alpha-\gamma+1} F(\alpha-\gamma+1, \beta-\gamma+1,2-\gamma ; \mu), \\
(-\mu)^{\alpha-\beta} g_{1}\left(\beta, \beta-\gamma+1,1+\beta-\alpha ; \frac{1}{\mu}\right)= \\
(-1)^{\alpha-\beta+1} \frac{\Gamma(1+\beta-\alpha) \Gamma(1-\beta) \Gamma(\alpha-\gamma+1)}{\Gamma(2-\gamma)}(-\mu)^{\alpha-\gamma+1} F(\alpha-\gamma+1, \beta-\gamma+1,2-\gamma ; \mu) .
\end{gathered}
$$


Here $|\arg (-\mu)|<\pi, \ln (-\mu)>0$ for $-\infty<\mu<-1$. The branch cut in the $\mu$-plane is $[0,+\infty)$. As for the minus signs, we choose $-\mu=e^{-i \pi} \mu$, then $0<\arg \mu<2 \pi$. Moreover, in our case $(-1)^{\alpha-\beta}=1$. With this preparation, we can write the connection matrix:

$$
\left[\varphi_{1}^{(\infty)}, \varphi_{2}^{(\infty)}\right]=\left[\varphi_{1}^{(0)}, \varphi_{2}^{(0)}\right] C_{\infty 0}, \quad 0<\arg \mu<2 \pi
$$

where $C_{\infty 0}$ is $(35)$.

In order to write $\Psi_{I N}^{M a t c h}$ in terms of the $\varphi_{i}^{(\infty)}$, let us compute the behavior for $\mu \rightarrow \infty$ of $\mu^{\theta_{0} / 2}(\mu-1)^{\theta_{x} / 2}\left(\begin{array}{cc}\varphi_{1}^{(\infty)} & \varphi_{2}^{(\infty)} \\ \xi_{1}^{(\infty)} & \xi_{2}^{(\infty)}\end{array}\right)$. We have:

$$
\begin{gathered}
\mu^{\frac{\theta_{0}}{2}}(\mu-1)^{\frac{\theta_{x}}{2}} \varphi_{1}^{(\infty)}=-\frac{32}{\left[\left(\theta_{0}+\theta_{x}\right)^{2}-1\right]\left[\left(\theta_{0}-\theta_{x}\right)^{2}-1\right]} \mu^{\frac{1}{2}}\left[1+O\left(\frac{1}{\mu}\right)\right]+\mu^{-\frac{3}{2}} \ln \mu\left[1+O\left(\frac{1}{\mu}\right)\right] . \\
\mu^{\frac{\theta_{0}}{2}}(\mu-1)^{\frac{\theta_{x}}{2}} \varphi_{2}^{(\infty)}=\mu^{-\frac{3}{2}}\left[1+O\left(\frac{1}{\mu}\right)\right] . \\
\mu^{\frac{\theta_{0}}{2}}(\mu-1)^{\frac{\theta_{x}}{2}} \xi_{1}^{(\infty)}=-\frac{2}{r} \mu^{-\frac{1}{2}} \ln \mu\left[1+O\left(\frac{1}{\mu}\right)\right]+O\left(\mu^{-\frac{1}{2}}\right) . \\
\mu^{\frac{\theta_{0}}{2}}(\mu-1)^{\frac{\theta_{x}}{2}} \xi_{2}^{(\infty)}=\frac{2}{r} \mu^{-\frac{1}{2}}\left[1+O\left(\frac{1}{\mu}\right)\right] .
\end{gathered}
$$

Therefore:

$$
\mu^{\frac{\theta_{0}}{2}}(\mu-1)^{\frac{\theta_{x}}{2}}\left(\begin{array}{ll}
\varphi_{1}^{(\infty)} & \varphi_{2}^{(\infty)} \\
\xi_{1}^{(\infty)} & \xi_{2}^{(\infty)}
\end{array}\right)=\left[I+O\left(\frac{1}{\mu}\right)\right] \mu^{\frac{1}{2} \sigma_{3}}\left(\begin{array}{cc}
1 & 0 \\
R \ln \mu & 1
\end{array}\right)\left(\begin{array}{cc}
-\frac{2}{r R} & 0 \\
0 & \frac{2}{r}
\end{array}\right) .
$$

We conclude that the matrix $\Psi_{0}$ used in the $\Psi_{O U T} \leftrightarrow \Psi_{I N}$ matching is:

$$
\Psi_{0}=\mu^{\frac{\theta_{0}}{2}}(\mu-1)^{\frac{\theta_{x}}{2}}\left(\begin{array}{ll}
\varphi_{1}^{(\infty)} & \varphi_{2}^{(\infty)} \\
\xi_{1}^{(\infty)} & \xi_{2}^{(\infty)}
\end{array}\right) C, \quad C:=\left(\begin{array}{cc}
-R \frac{r}{2} & 0 \\
0 & \frac{r}{2}
\end{array}\right)=\left(\begin{array}{cc}
\frac{\left[1-\left(\theta_{0}+\theta_{x}\right)^{2}\right]\left[\left(\theta_{0}-\theta_{x}\right)^{2}-1\right]}{32} & 0 \\
0 & \frac{r}{2}
\end{array}\right) .
$$

As we have already remarked, we do the transformation $\Psi_{I N}^{M a t c h} \mapsto \Psi_{I N}^{M a t c h} C_{I N}{ }^{-1}$, to compute the $x$-independent monodromy at $\lambda=0, x$. This means that we have to compute the monodromy of:

$$
\begin{gathered}
\Psi_{I N}^{\text {Match }} C_{I N}{ }^{-1}=K_{0}(x) \Psi_{0}(\lambda / x)= \\
=K_{0}(x)\left(\frac{\lambda}{x}\right)^{\frac{\theta_{0}}{2}}\left(\frac{\lambda}{x}-1\right)^{\frac{\theta_{x}}{2}}\left(\begin{array}{ll}
\varphi_{1}^{(\infty)} & \varphi_{2}^{(\infty)} \\
\xi_{1}^{(\infty)} & \xi_{2}^{(\infty)}
\end{array}\right) C, \\
=K_{0}(x)\left(\frac{\lambda}{x}\right)^{\frac{\theta_{0}}{2}}\left(\frac{\lambda}{x}-1\right)^{\frac{\theta_{x}}{2}}\left(\begin{array}{cc}
\varphi_{1}^{(0)} & \varphi_{2}^{(0)} \\
\xi_{1}^{(0)} & \xi_{2}^{(0)}
\end{array}\right) C_{\infty 0} C, \\
=K_{0}(x)\left(\frac{\lambda}{x}\right)^{\frac{\theta_{0}}{2}}\left(\frac{\lambda}{x}-1\right)^{\frac{\theta_{x}}{2}}\left(\begin{array}{cc}
\varphi_{1}^{(1)} & \varphi_{2}^{(1)} \\
\xi_{1}^{(1)} & \xi_{2}^{(1)}
\end{array}\right) C_{01} C_{\infty 0} C .
\end{gathered}
$$

In order to do this, we observe that from the definition of $\varphi_{1}^{(0)}, \varphi_{2}^{(0)}$ if follows that:

$$
K_{0}(x)\left(\frac{\lambda}{x}\right)^{\frac{\theta_{0}}{2}}\left(\frac{\lambda}{x}-1\right)^{\frac{\theta_{x}}{2}}\left(\begin{array}{ll}
\varphi_{1}^{(0)} & \varphi_{2}^{(0)} \\
\xi_{1}^{(0)} & \xi_{2}^{(0)}
\end{array}\right)=\psi_{0}^{I N}(x)(I+O(\lambda)) \lambda^{\frac{\theta_{0}}{2} \sigma_{3}}
$$

for $\lambda \rightarrow 0$. From the definition of $\varphi_{1}^{(1)}, \varphi_{2}^{(1)}$, it follows that:

$$
K_{0}(x)\left(\frac{\lambda}{x}\right)^{\frac{\theta_{0}}{2}}\left(\frac{\lambda}{x}-1\right)^{\frac{\theta_{x}}{2}}\left(\begin{array}{ll}
\varphi_{1}^{(1)} & \varphi_{2}^{(1)} \\
\xi_{1}^{(1)} & \xi_{2}^{(1)}
\end{array}\right)=\psi_{x}^{I N}(x)(I+O(\lambda-x))(\lambda-x)^{\frac{\theta_{x}}{2} \sigma_{3}},
$$


for $\lambda \rightarrow x$. It is not necessary here to write explicitly the invertible matrices $\psi_{0}^{I N}(x)$ and $\psi_{x}^{I N} x$. The above construction implies that $\Psi_{I N}^{M a t c h} C_{I N}{ }^{-1}$ has monodromy matrices at $\lambda=0, x$ respectively given by:

$$
\begin{gathered}
M_{0}=C^{-1}\left(C_{\infty 0}\right)^{-1} \exp \left\{i \pi \theta_{0} \sigma_{3}\right\} C_{\infty 0} C, \\
M_{x}=C^{-1}\left(C_{\infty 0}\right)^{-1}\left(C_{01}\right)^{-1} \exp \left\{i \pi \theta_{x} \sigma_{3}\right\} C_{01} C_{\infty 0} C .
\end{gathered}
$$

These matrices coincide with the monodromy matrices of $\Psi C_{I N}{ }^{-1}$.

As a last simplification, we consider the transformation $M \mapsto C M C^{-1}$. We obtain the result of theorem 3, case c). Namely:

$$
\begin{gathered}
M_{0}=\left(C_{\infty 0}\right)^{-1} \exp \left\{i \pi \theta_{0} \sigma_{3}\right\} C_{\infty 0}, \quad M_{\infty}=\left(\begin{array}{cc}
-1 & 0 \\
2 \pi i\left(1-\frac{r_{1}}{\rho}\right) & -1
\end{array}\right), \\
M_{1}=\left(\begin{array}{cc}
1 & 0 \\
2 \pi i \frac{r_{1}}{\rho} & 1
\end{array}\right), \quad M_{x}=\left(C_{\infty 0}\right)^{-1}\left(C_{01}\right)^{-1} \exp \left\{i \pi \theta_{x} \sigma_{3}\right\} C_{01} C_{\infty 0} .
\end{gathered}
$$

We observe that $r$ does not appear in the monodromy matrices.

\section{Appendix 1}

Proposition 2 Let $B_{0}, B_{1}$ be $2 \times 2$ matrices such that

$$
\text { Eigenvalues }\left(B_{0}\right)=0,-c, \quad \text { Eigenvalues }\left(B_{1}\right)=0, c-a-b \text {. }
$$

and $B_{0}+B_{1}$ is either diagonalizable:

$$
\left.B_{0}+B_{1}=\left(\begin{array}{cc}
-a & 0 \\
0 & -b
\end{array}\right) \quad \text { (it may happen that } a=b\right),
$$

or it is a Jordan form:

$$
B_{0}+B_{1}=\left(\begin{array}{cc}
-a & 1 \\
0 & -a
\end{array}\right) .
$$

Then, $B_{0}$ and $B_{1}$ can be computed as in the following cases. Let $r, s$ be any complex numbers.

\section{1) Diagonalizable case.}

Case $a \neq b$ :

$$
\begin{gathered}
B_{0}:=\left(\begin{array}{cc}
\frac{a(b-c)}{a-b} & r \\
\frac{a b(a-c)(c-b)}{r(a-b)^{2}} & \frac{b(c-a)}{a-b}
\end{array}\right), \quad B_{1}=\left(\begin{array}{cc}
\frac{a(c-a)}{a-b} & -r \\
-\left(B_{0}\right)_{21} & \frac{b(b-c)}{a-b}
\end{array}\right), \quad r \neq 0 \\
\text { if } a=0: \quad B_{0}=\left(\begin{array}{cc}
0 & r \\
0 & -c
\end{array}\right), \quad B_{1}=\left(\begin{array}{cc}
0 & -r \\
0 & c-b
\end{array}\right) . \\
\text { If } b=0: \quad B_{0}=\left(\begin{array}{cc}
-c & r \\
0 & 0
\end{array}\right), \quad B_{1}=\left(\begin{array}{cc}
c-a & -r \\
0 & 0
\end{array}\right) . \\
\text { If } a=c \neq b: \quad B_{0}=\left(\begin{array}{cc}
-a & r \\
0 & 0
\end{array}\right), \quad B_{1}=\left(\begin{array}{cc}
0 & -r \\
0 & -b
\end{array}\right) . \\
\text { If } b=c \neq a: \quad B_{0}=\left(\begin{array}{cc}
0 & r \\
0 & -b
\end{array}\right), \quad B_{1}=\left(\begin{array}{cc}
-a & -r \\
0 & 0
\end{array}\right) .
\end{gathered}
$$

Cases (99)-(102) are already included in (98).

Case $a=b$. We have two sub-cases:

$$
\begin{gathered}
\text { If } a=b=c: \quad B_{0}=\left(\begin{array}{cc}
-c-s & r \\
-\frac{s(c+s)}{r} & s
\end{array}\right), \quad B_{1}=\left(\begin{array}{cc}
s & -r \\
\frac{s(c+s)}{r} & -c-s
\end{array}\right) . \\
\text { If } a=b=0: \quad B_{0}=\left(\begin{array}{cc}
-c-s & r \\
-\frac{s(c+s)}{r} & s
\end{array}\right), \quad B_{1}=-B_{0} .
\end{gathered}
$$


The transpose matrices of all the above cases are also possible.

\section{2) Jordan case.}

For $a \neq 0$ and $a \neq c$ we have:

$$
B_{0}=\left(\begin{array}{cc}
r & \frac{r(r+c)}{a(a-c)} \\
a(c-a) & -c-r
\end{array}\right), \quad B_{1}=\left(\begin{array}{cc}
-a-r & 1-\frac{r(r+c)}{a(a-c)} \\
a(a-c) & c-a+r
\end{array}\right) .
$$

For $a=0$, or $a=c$, we have two possibilities:

$$
B_{0}=\left(\begin{array}{cc}
0 & r \\
0 & -c
\end{array}\right), \quad B_{1}=\left(\begin{array}{cc}
-a & 1-r \\
0 & -a+c
\end{array}\right)
$$

or

$$
B_{0}=\left(\begin{array}{cc}
-c & r \\
0 & 0
\end{array}\right), \quad B_{1}=\left(\begin{array}{cc}
c-a & 1-r \\
0 & -a
\end{array}\right)
$$

Proposition 3 Let $B_{0}$ and $B_{1}$ be as in Proposition 2. The linear system:

$$
\frac{d}{d z}\left(\begin{array}{l}
\varphi \\
\xi
\end{array}\right)=\left[\frac{B_{0}}{z}+\frac{B_{1}}{z-1}\right]\left(\begin{array}{l}
\varphi \\
\xi
\end{array}\right)
$$

may be reduced to a Gauss hyper-geometric equation, in the following cases.

Diagonalizable case (i.e. from (98) to (104)):

$$
z(1-z) \frac{d^{2} \varphi}{d z^{2}}+(1+c-(a+[b+1]+1) z) \frac{d \varphi}{d z}-a(b+1) \varphi=0 .
$$

The component $\xi$ is obtained by the following equalities, according to the different cases of Proposition 2.

Cases (98) (99) (100) (101) (102):

$$
\xi=\frac{1}{r}\left[z(1-z) \frac{d \varphi}{d z}-a\left(z+\frac{b-c}{a-b}\right) \varphi\right]
$$

Case (103):

$$
\xi=\frac{1}{r}\left[z(1-z) \frac{d \varphi}{d z}+(c+s-c z) \varphi\right]
$$

Case (104):

$$
\xi=\frac{1}{r}\left[z(1-z) \frac{d \varphi}{d z}+(c+s) \varphi\right]
$$

Jordan case (105): The equation for $\varphi$ is in Gauss hypergeometic form only when $r=-a$. In this case, the matrices (105) are:

$$
B_{0}=\left(\begin{array}{cc}
-a & 1 \\
a(c-a) & a-c
\end{array}\right), \quad B_{1}=\left(\begin{array}{cc}
0 & 0 \\
a(a-c) & c-2 a
\end{array}\right) .
$$

The equation is:

$$
\begin{gathered}
z(1-z) \frac{d^{2} \varphi}{d z^{2}}+[1+c-(2 a+1) z] \frac{d \varphi}{d z}-a^{2} \varphi=0 \\
\xi=z \frac{d \varphi}{d z}+a \varphi
\end{gathered}
$$

Jordan case (106): for $r=1$ we get:

$$
\text { For } a=0, \quad z(1-z) \frac{d^{2} \varphi}{d z^{2}}+(1+c-z) \frac{d \varphi}{d z}=0 .
$$




$$
\text { For } c=a, \quad z(1-z) \frac{d^{2} \varphi}{d z^{2}}+(1+a-(2 a+1) z) \frac{d \varphi}{d z}+\left(-a^{2}-\frac{a}{1-z}\right) \varphi=0 .
$$

The Gauss form appears only when $a=0$.

Jordan case (10\%): for $r=1$ we get:

$$
\begin{aligned}
& \text { For } a=0, \quad z(1-z) \frac{d^{2} \varphi}{d z^{2}}+(1+c-z) \frac{d \varphi}{d z}+\frac{c}{1-z} \varphi=0 . \\
& \text { For } c=a, \quad z(1-z) \frac{d^{2} \varphi}{d z^{2}}+(1+a-(2 a+1) z) \frac{d \varphi}{d z}-a^{2} \varphi=0 .
\end{aligned}
$$

For $r \neq 1$, we don't get a Gauss hyper-geometric form for the equation of $\varphi$ in both cases (106) and (10\%). Nevertheless, the matrices are in upper triangular form, so the equation for $\xi$ is solvable by elementary integration.

Jordan case (105): The equation for $\xi$ is in Gauss hypergeometic form:

$$
\begin{gathered}
z(z-1) \frac{d^{2} \xi}{d z^{2}}+(1+c-2(a+1) z) \frac{d \xi}{d z}-a(a+1) \xi=0, \\
\varphi(z)=\frac{1}{a(a-c)}\left[z(z-1) \frac{d \xi}{d z}+(a z-c-r) \xi\right] .
\end{gathered}
$$

As a compendium to the above proposition, we recall that any irreducible representation of $\pi_{1}\left(\mathbf{C P}^{1} \backslash\{0,1, \infty\}\right) \mapsto G L(2, \mathbf{C})$ can be realized as the monodromy group of a Riemann (or Gauss) equation. A reducible representation (namely, $M_{0}, M_{1}, M_{\infty}$ are in upper triangular form) can be realized by the monodromy of a $2 \times 2$ Fuchsian system

$$
\frac{d Y}{d z}=\left[\frac{B_{0}}{z}+\frac{B_{1}}{z-1}\right] Y
$$

where $B_{0}, B_{1}$ are $2 \times 2$ upper triangular matrices. We also state the following:

Lemma 12 Consider a $2 \times 2$ linear system:

$$
\frac{d Y(z)}{d z}=A(z) Y, \quad A(z)=\left(\begin{array}{cc}
a(z) & b(z) \\
0 & c(z)
\end{array}\right)
$$

such that $A(z)$ is meromorphic, with poles $a_{1}, a_{2}, \ldots, a_{N}, \infty$. The monodromy group is generated by $N$ upper triangular monodromy matrices:

$$
M_{i}=\left(\begin{array}{cc}
\lambda_{1}^{(i)} & R^{(i)} \\
0 & \lambda_{2}^{(i)}
\end{array}\right), \quad i=1,2, \ldots, N
$$

where $\lambda_{1}^{(i)}, \lambda_{2}^{(i)}, R^{(i)}$ are constants (i.e. they are independent of $z$ ) given by:

$$
\begin{gathered}
\lambda_{1}^{(i)}=\exp \left\{\left.2 \pi i \operatorname{Res} a(z)\right|_{a_{i}}\right\}, \quad \lambda_{2}^{(i)}=\exp \left\{\left.2 \pi i \operatorname{Res} c(z)\right|_{a_{i}}\right\} \\
R^{(i)}=\int_{z}^{z \exp \{2 \pi i\}} d s b(s) \frac{u_{2}(s)}{u_{1}(s)}+\left(\lambda_{1}^{(i)}-\lambda_{2}^{(i)}\right) \int_{z_{0}}^{z} d s b(s) \frac{u_{2}(s)}{u_{1}(s)} .
\end{gathered}
$$

$R^{(i)}$ depends on a chosen non-singular base point $z_{0}$, but not on $z$. One of the non zero $R^{(i)}$ can be put equal to 1 , by re-defining $Y \mapsto Y C, C=\left(\begin{array}{cc}1 & 0 \\ 0 & 1 / R^{(i)}\end{array}\right)$.

Proof: Let us write $Y=\left(\begin{array}{l}y_{1} \\ y_{2}\end{array}\right)$ and the equation in the form:

$$
\frac{d y_{1}}{d z}=a(z) y_{1}+b(z) y_{2}, \quad \frac{d y_{2}}{d z}=c(z) y_{2} .
$$


Let $z_{0} \neq a_{i}, \infty, i=1, \ldots, N$. The second equation has solution $y_{2}(z)=C_{2} u_{2}$, where $u_{2}:=$ $\exp \left\{\int_{z_{0}}^{z} d s c(s)\right\}, C_{2} \in \mathbf{C}$. The first equation becomes:

$$
\frac{d y_{1}}{d z}=a(z) y_{1}+C_{2} b(z) u_{2}(z)
$$

We solve the first equation by variation of parameters. Let $u_{1}(z)=\exp \left\{\int_{z_{0}}^{z} d s a(s)\right\}$ be a fundamental solution of the homogeneous equation $\frac{d y_{1}}{d z}=a(z) y_{1}$. We look for a solution of (110) of the form $y_{2}(z)=w(z) u_{1}(z)$. Substitution gives:

$$
\frac{d w}{d z}=C_{2} b(z) \frac{u_{2}(z)}{u_{1}(z)} \Longrightarrow w(z)=C_{2} v(z)+C_{1}, \quad C_{1} \in \mathbf{C} .
$$

where:

$$
v(z)=\int_{z_{0}}^{z} d s b(s) \frac{u_{2}(s)}{u_{1}(s)}
$$

The general solution of (110) is $y_{1}(z)=C_{1} u_{1}(z)+C_{2} v(z) u_{1}(z)$. Then, a fundamental solution for the initial system can be chosen to be:

$$
Y(z)=\left(\begin{array}{cc}
u_{1}(z) & v(z) u_{1}(z) \\
0 & u_{2}(z)
\end{array}\right)
$$

We compute the monodromy for $\left(z-a_{i}\right) \mapsto\left(z-a_{i}\right) e^{2 \pi i}$. We have:

$$
\begin{aligned}
& u_{1}(z) \mapsto \lambda_{1}^{(i)} u_{1}(z), \quad \lambda_{1}^{(i)}=\exp \left\{2 \pi i \operatorname{Res} a(z)||_{a_{i}}\right\}, \\
& u_{2}(z) \mapsto \lambda_{2}^{(i)} u_{1}(z), \quad \lambda_{2}^{(i)}=\exp \left\{\left.2 \pi i \operatorname{Res} c(z)\right|_{a_{i}}\right\} .
\end{aligned}
$$

By linearity, the vector solution $\left(\begin{array}{c}v(z) u_{1}(z) \\ u_{2}(z)\end{array}\right)$ is transformed into a linear combination of two independent vector solutions: $R^{(i)}\left(\begin{array}{c}u_{1}(z) \\ 0\end{array}\right)+S^{(i)}\left(\begin{array}{c}v(z) u_{1}(z) \\ u_{2}(z)\end{array}\right)$. Moreover, $S^{(i)}$ must coincide with $\lambda_{2}^{(i)}$, because - by virtue of $(111)-u_{2}(z) \mapsto \lambda_{2}^{(i)} u_{2}(z)$. Namely:

$$
\left(\begin{array}{c}
v(z) u_{1}(z) \\
u_{2}(z)
\end{array}\right) \mapsto R^{(i)}\left(\begin{array}{c}
u_{1}(z) \\
0
\end{array}\right)+\lambda_{2}^{(i)}\left(\begin{array}{c}
v(z) u_{1}(z) \\
u_{2}(z)
\end{array}\right), \quad R^{(i)} \in \mathbf{C} .
$$

Thus:

$$
\begin{aligned}
Y(z)=\left(\begin{array}{cc}
u_{1}(z) & v(z) u_{1}(z) \\
0 & u_{2}(z)
\end{array}\right) & \mapsto\left(\begin{array}{cc}
\lambda_{1}^{(i)} u_{1}(z) & \lambda_{2}^{(i)} v(z) u_{1}(z)+R^{(i)} u_{1}(z) \\
0 & \lambda_{2}^{(i)} u_{2}(z)
\end{array}\right)= \\
& =Y(z)\left(\begin{array}{cc}
\lambda_{1}^{(i)} & R^{(i)} \\
0 & \lambda_{2}^{(i)}
\end{array}\right) .
\end{aligned}
$$

Let $C_{i}$ be a small loop around $a_{i}$. To find $R^{(i)}$, let us observe that $u_{1}(z) v(z) \mapsto \lambda_{1}^{(i)} u_{1}(z) \tilde{v}(z)$, where: $\tilde{v}(z)=v(z)+K_{i}(z), K_{i}(z):=\int_{z}^{z \exp \{2 \pi i\}} d s b(s) \frac{u_{2}(s)}{u_{1}(s)}$. Thus:

$$
Y(z)=\left(\begin{array}{cc}
u_{1}(z) & v(z) u_{1}(z) \\
0 & u_{2}(z)
\end{array}\right) \mapsto\left(\begin{array}{cc}
\lambda_{1}^{(i)} u_{1}(z) & \lambda_{1}^{(i)} v(z) u_{1}(z)+K_{i}(z) u_{1}(z) \\
0 & \lambda_{2}^{(i)} u_{2}(z)
\end{array}\right) .
$$

We must have: $\lambda_{1}^{(i)} v(z) u_{1}(z)+K_{i}(z) u_{1}(z) \equiv \lambda_{2}^{(i)} v(z) u_{1}(z)+R^{(i)} u_{1}(z)$; namely:

$$
R^{(i)}=K_{i}(z)+\left(\lambda_{1}^{(i)}-\lambda_{2}^{(i)}\right) v(z) .
$$




\section{Appendix 2: Formal Asymptotic Expansion}

1) We consider systems (62):

$$
\frac{d Y}{d z}=\left[\Omega+\frac{D_{1}}{z}+\sum_{n=2}^{\infty} \frac{D_{n}}{z^{n}}\right] Y:=D(z) Y, \quad \Omega=\operatorname{diag}\left(\omega_{1}, \omega_{2}, \ldots, \omega_{n}\right),
$$

with $\omega_{i} \neq \omega_{j}$, for $i \neq j$. We introduce a gauge transformation $Y=G(z) \tilde{Y}$, such that:

$$
\frac{\tilde{Y}}{d z}=\left[G^{-1}(z) D(z) G(z)-G^{-1}(z) \frac{d G(z)}{d z}\right] \tilde{Y}
$$

be in the simple form:

$$
\frac{d \tilde{Y}}{d z}=\left[\Omega+\frac{\Omega_{1}}{z}\right] \tilde{Y}, \quad \Omega, \Omega_{1} \text { diagonal. }
$$

Formally, we write $G(z)$ as:

$$
G(z)=I+\frac{G_{1}}{z}+\frac{G_{2}}{z^{2}}+\ldots, \quad z \rightarrow \infty .
$$

If the $G_{n}$ 's can be determined, we get the formal solution:

$$
Y(z) \sim\left[I+\sum_{n=1}^{\infty} \frac{G_{n}}{z^{n}}\right] \exp \left\{z \Omega+\Omega_{1} \ln (z)\right\}, \quad z \rightarrow \infty
$$

For a sector of angular with $\pi+\epsilon, \epsilon>0$ sufficiently small (but finite and non zero), there exists a unique solution $Y(z)$ with the above asymptotic expansion [3].

In order to determine $G_{n}$ and $\Omega_{1}$, we solve $D(z) G(z)-\partial_{z} G(z)=G(z)\left(\Omega_{0}+\Omega_{1} z^{-1}\right)$ :

$$
\left(\Omega+\sum_{n=1}^{\infty} \frac{D_{n}}{z^{n}}\right)\left(I+\sum_{n=1}^{\infty} \frac{G_{n}}{z^{n}}\right)+\sum_{n=2}^{\infty} \frac{(n-1) G_{n-1}}{z^{n}}=\left(I+\sum_{n=1}^{\infty} \frac{G_{n}}{z^{n}}\right)\left(\Omega+\frac{\Omega_{1}}{z}\right) .
$$

We identify equal powers of $z^{-1}$. From the power $1 / z$ we get:

$$
\Omega_{1}=\operatorname{diag}\left(\omega_{1}^{(1)}, \ldots, \omega_{n}^{(1)}\right), \quad \omega_{i}^{(1)}:=\left(\Omega_{1}\right)_{i i}=\left(D_{1}\right)_{i i}, \quad\left(G_{1}\right)_{i j}=-\frac{\left(D_{1}\right)_{i j}}{\omega_{i}-\omega_{j}} .
$$

From the power $1 / z^{2}$, for $i \neq j$, we compute $\left(G_{2}\right)_{i j}$, and for $i=j$ we compute:

$$
\left(G_{1}\right)_{i i}=-\left(D_{2}\right)_{i i}-\sum_{k \neq i}\left(D_{1}\right)_{i k}\left(G_{1}\right)_{k i} .
$$

From the power $1 / z^{n}$ we get:

$$
\begin{aligned}
&\left(G_{n-1}\right)_{i i}=\frac{1}{n-1}\{\left.-\left(D_{n}+D_{n-1} G_{1}+\ldots+D_{2} G_{n-2}\right)_{i i}-\sum_{k \neq i}\left(D_{1}\right)_{i k}\left(G_{n-1}\right)_{k i}\right\}, \\
&\left(G_{n}\right)_{i j}=\frac{1}{\omega_{i}-\omega_{j}}\left\{\left[\omega_{j}^{(1)}-\omega_{i}^{(1)}-(n-1)\right]\left(G_{n-1}\right)_{i j}-\sum_{k \neq i}\left(D_{1}\right)_{i k}\left(G_{n-1}\right)_{k j}-\right. \\
&\left.-\left(D_{n}+D_{n-1} G_{1}+\ldots+D_{2} G_{n-2}\right)_{i j}\right\}, \quad i \neq j
\end{aligned}
$$

2) We consider the system (63):

$$
\frac{d Y}{d z}=\left[x^{2} \Lambda z+x \Lambda+\frac{E_{1}}{z}+\sum_{n=2}^{\infty} \frac{E_{n}}{z^{n}}\right] Y:=E(z) Y, \quad \Lambda=\operatorname{diag}\left(\lambda_{1}, \lambda_{2}, \ldots, \lambda_{n}\right) .
$$


with $\lambda_{i} \neq \lambda_{j}$ for $i \neq j$. We introduce a gauge transformation:

$$
Y(z)=K(z) \tilde{Y}(z)
$$

in order to reach the simple form:

Formally, we write:

$$
\begin{aligned}
& \frac{d \tilde{Y}}{d z}=\left[K^{-1}(z) E(z) K(z)-K^{-1}(z) \frac{d K(z)}{d z}\right] \tilde{Y} \\
& \equiv\left[x^{2} \Lambda z+x \Lambda+\frac{\Lambda_{1}}{z}\right] \tilde{Y}, \quad \Lambda_{1} \text { diagonal. }
\end{aligned}
$$

$$
K(z) \sim I+\frac{K_{1}}{z}+\frac{K_{2}}{z}+\ldots=I+\sum_{n=1}^{\infty} \frac{K_{n}}{z} .
$$

Provided that we can determine the matrices $K_{n}$, we obtain the formal solution:

$$
Y(z) \sim\left[I+\sum_{n=1}^{\infty} \frac{K_{n}}{z^{n}}\right] \exp \left\{\frac{x^{2}}{2} \Lambda z^{2}+x \Lambda z \Lambda_{1} \ln x\right\}
$$

For a sector of angular with $\frac{\pi}{2}+\epsilon, \epsilon>0$ sufficiently small (but finite and non zero), there exists a unique solution $Y(z)$ with the above asymptotic expansion [3].

In order to determine $K_{n}$ and $\Lambda_{1}$, we solve $E(z) K(z)-\partial_{z} K(z)=K(z)\left(x^{2} \Lambda z^{2}+\Lambda z+\Omega_{1} z^{-1}\right)$ :

$$
\begin{aligned}
\left(x^{2} \Lambda z^{2}+\Lambda z+\sum_{n=1}^{\infty} \frac{E_{n}}{z^{n}}\right)(I+ & \left.\sum_{n=1}^{\infty} \frac{K_{n}}{z^{n}}\right)+\sum_{n=2}^{\infty} \frac{(n-1) K_{n-1}}{z^{n}}= \\
& =\left(I+\sum_{n=1}^{\infty} \frac{K_{n}}{z^{n}}\right)\left(x^{2} \Lambda z^{2}+\Lambda z+\frac{\Omega_{1}}{z}\right) .
\end{aligned}
$$

We identify equal powers of $z^{-1}$.

Power $z$. It is an identity: $x^{2} \Lambda=x^{2} \Lambda$.

Power $z^{0}$.

$$
x \Lambda+x^{2} \Lambda K_{1}=x \Lambda+x^{2} K_{1} \Lambda \quad \Longrightarrow \quad\left[\Lambda, K_{1}\right]=0
$$

This means that $K_{1}$ is a diagonal matrix.

Power $1 / z$. We obtain the equation:

$$
x^{2}\left[\Lambda, K_{2}\right]=\Lambda_{1}-E_{1} \quad \Longrightarrow \quad\left(\Lambda_{1}\right)_{i i}=\left(E_{1}\right)_{i i}, \quad\left(G_{2}\right)_{i j}=-\frac{\left(E_{1}\right)_{i j}}{x^{2}\left(\lambda_{i}-\lambda_{j}\right)}, \quad i \neq j .
$$

Power $1 / z^{2}$. We obtain the equation:

$$
x^{2}\left[\Lambda, K_{3}\right]=x\left[K_{2}, \Lambda\right]+K_{1} \Lambda_{1}-E_{1} K_{1}-K_{1}-E_{2} \equiv \frac{E_{1}-\Lambda_{1}}{x}+K_{1} \Lambda_{1}-E_{1} K_{1}-K_{1}-E_{2}
$$

Thus:

$$
\begin{gathered}
\left(K_{1}\right)_{i i}=-\left(E_{2}\right)_{i i} \\
\left(K_{3}\right)_{i j}=\frac{1}{x^{2}\left(\lambda_{i}-\lambda_{j}\right)}\left[\left(E_{1}\right)_{i j}\left(\frac{1}{x}+\left(E_{2}\right)_{j j}\right)-\left(E_{2}\right)_{i j}\right], \quad i \neq j .
\end{gathered}
$$

Power $1 / z^{2}$. We obtain the equation:

$$
x^{2}\left[\Lambda, K_{4}\right]=x\left[K_{3}, \Lambda\right]+K_{2} \Lambda_{1}-E_{1} K_{2}-E_{2} K_{1}-2 K_{2}-E_{3}
$$

The diagonal part gives:

$$
2\left(K_{2}\right)_{i j}=\left[\left(E_{2}\right)_{i i}\right]^{2}-\left(E_{3}\right)_{i i}-\sum_{k \neq i}\left(E_{1}\right)_{i k}\left(K_{2}\right)_{k i} .
$$

The non diagonal part gives $\left(K_{4}\right)_{i j}, i \neq j$.

We content ourselves with these results, namely the determination of $K_{1}$ and $K_{2}$. With the same procedure, we can determine all the $K_{n}$ 's. 


\section{Appendix 3: Birational Transformations}

All the solutions of (PVI) of the form:

$$
y(x)=b_{0}+b_{1} x+b_{2} x^{2}+\ldots+b_{N} x^{N}+\ldots
$$

are obtained from the matching procedure of sections 5, 6, 7. By this we mean that solutions of type (112) are the solutions given by the matching procedure, or they can be obtained from solutions given by the matching procedure via one of the birational transformations of [24] and the transformation $(22)$.

Birational transformations are symmetries of (PVI), namely invertible transformations:

$$
y^{\prime}(x)=\frac{P(x, y(x))}{Q(x, y(x))}, \quad x^{\prime}=\frac{p(x)}{q(x)}, \quad\left(\theta_{0}, \theta_{x}, \theta_{1}, \theta_{\infty}\right) \mapsto\left(\theta_{0}^{\prime}, \theta_{x}^{\prime}, \theta_{1}^{\prime}, \theta_{\infty}^{\prime}\right)
$$

such that $y(x)$ satisfies (PVI) with coefficients $\theta_{0}, \theta_{x}, \theta_{1}, \theta_{\infty}$ and variable $x$, if and only if $y^{\prime}\left(x^{\prime}\right)$ satisfies (PVI) with coefficients $\theta_{0}^{\prime}, \theta_{x}^{\prime}, \theta_{1}^{\prime}, \theta_{\infty}^{\prime}$ and variable $x^{\prime}$. The functions $P, Q$ are polynomials; $p, q$ are linear; the transformation of the $\theta_{\mu}$ 's is an element of a linear representation of one of the following groups. Permutation group; the Weyl group of the root system $D_{4}$; the group of translations $v:=\left(v_{1}, v_{2}, v_{3}, v_{4}\right) \mapsto v+e_{j}, \mathrm{j}=1,2,3,4$ (where $\left.e_{1}=(1,0,0,0), \ldots, e_{4}=(0,0,0,1)\right)$. 7

* Permutation group:

$$
\begin{aligned}
& x^{1}: \quad \theta_{1}^{\prime}=\theta_{0}, \quad \theta_{0}^{\prime}=\theta_{1} ; \quad \theta_{x}^{\prime}=\theta_{x}, \quad \theta_{\infty}^{\prime}=\theta_{\infty} ; \quad y^{\prime}(x)=1-y(x), \quad x=1-x^{\prime} . \\
& x^{2}: \quad \theta_{0}^{\prime}=\theta_{\infty}-1, \quad \theta_{\infty}^{\prime}=\theta_{0}+1 ; \quad \theta_{1}^{\prime}=\theta_{1}, \quad \theta_{x}^{\prime}=\theta_{x} ; \quad y^{\prime}(x)=\frac{1}{y(x)}, \quad x=\frac{1}{x^{\prime}} . \\
& x^{3}: \quad \theta_{0}^{\prime}=\theta_{x}, \quad \theta_{x}^{\prime}=\theta_{0} ; \quad \theta_{1}^{\prime}=\theta_{1}, \quad \theta_{\infty}^{\prime}=\theta_{\infty} ; \quad y^{\prime}(x)=\frac{x-y(x)}{x-1}, \quad x=\frac{x^{\prime}}{x^{\prime}-1} .
\end{aligned}
$$

* Weyl Group:

$w_{1}:$

$$
\theta_{1}^{\prime}=-\theta_{1} ; \quad \theta_{0}^{\prime}=\theta_{0}, \quad \theta_{x}^{\prime}=\theta_{x}, \quad \theta_{\infty}^{\prime}=\theta_{\infty}
$$

$w_{2}$ :

$$
\begin{aligned}
\theta_{0}^{\prime}=\frac{\theta_{0}+\theta_{1}+\theta_{x}+\theta_{\infty}}{2}-1, & \theta_{1}^{\prime}=\frac{\theta_{0}+\theta_{1}-\theta_{x}-\theta_{\infty}}{2}+1, \\
\theta_{x}^{\prime}=\frac{\theta_{0}-\theta_{1}+\theta_{x}-\theta_{\infty}}{2}+1, & \theta_{\infty}^{\prime}=\frac{\theta_{0}-\theta_{1}-\theta_{x}+\theta_{\infty}}{2}+1
\end{aligned}
$$

$w_{3}:$

$$
\theta_{\infty}^{\prime}=2-\theta_{\infty} ; \quad \theta_{0}^{\prime}=\theta_{0}, \quad \theta_{x}^{\prime}=\theta_{x}, \quad \theta_{1}^{\prime}=\theta_{1}
$$

$w_{4}$ :

$$
\theta_{\infty}^{\prime}=2-\theta_{\infty} ; \quad \theta_{x}^{\prime}=2-\theta_{x} ; \quad \theta_{0}^{\prime}=\theta_{0}, \quad \theta_{1}^{\prime}=\theta_{1}
$$

The variable $x^{\prime}=x$, but $y^{\prime}(x)$ is quite complicated and will not be given here (see [24]).

$*$ Shift $l_{j}: v \mapsto v+e_{j}$ :

$$
\begin{aligned}
& l_{1}: \quad \theta_{0}^{\prime}=\theta_{0}+1, \quad \theta_{1}^{\prime}=\theta_{1}+1 ; \quad \theta_{x}^{\prime}=\theta_{x}, \quad \theta_{\infty}^{\prime}=\theta_{\infty} . \\
& l_{2}: \quad \theta_{0}^{\prime}=\theta_{0}+1, \quad \theta_{1}^{\prime}=\theta_{1}-1 ; \quad \theta_{x}^{\prime}=\theta_{x}, \quad \theta_{\infty}^{\prime}=\theta_{\infty} . \\
& l_{3}: \quad \theta_{x}^{\prime}=\theta_{x}+1, \quad \theta_{\infty}^{\prime}=\theta_{\infty}+1 ; \quad \theta_{0}^{\prime}=\theta_{0}, \quad \theta_{1}^{\prime}=\theta_{1} \text {. } \\
& l_{4}: \quad \theta_{x}^{\prime}=\theta_{x}+1, \quad \theta_{\infty}^{\prime}=\theta_{\infty}-1 ; \quad \theta_{0}^{\prime}=\theta_{0}, \quad \theta_{1}^{\prime}=\theta_{1} \text {. }
\end{aligned}
$$

The variable $x^{\prime}=x$, but $y^{\prime}(x)$ is quite complicated and will not be given here (see [24]).

\footnotetext{
${ }^{7}$ We note that $\theta_{1}, \theta_{x}, \theta_{0}$ are defined up to the sign. Moreover, $\theta_{\infty}$ is defined up to $\theta_{\infty} \mapsto 2-\theta_{\infty}$, and for this reason symmetries are described in [24] in terms of $\chi_{\infty}:=\theta_{\infty}-1$.
} 
For the Taylor solutions, we have $\sigma=\theta_{1}-\theta_{\infty}$. Denote $\sigma^{\prime}:=\theta_{1}^{\prime}-\theta_{\infty}^{\prime}$. If we start from a Taylor solution constructed in sections $5,6,7$ by means of the matching procedure, developed for $\sigma=\theta_{1}-\theta_{\infty}$, then the birational transformations allow to obtain the solutions defined for $\sigma= \pm\left(\theta_{1} \pm \backslash \mp \theta_{\infty}\right)+n, n \in \mathbf{Z}$. This is a consequence of the following actions:

$$
\begin{array}{cl}
l_{1} \text { and } l_{4}: \sigma \mapsto \sigma^{\prime}=\sigma+1 . & l_{2} \text { and } l_{3}: \sigma \mapsto \sigma^{\prime}=\sigma-1 \\
w_{3} \cdot l_{1} \cdot l_{1}: \sigma \mapsto \sigma^{\prime}=\theta_{1}+\theta_{\infty} . & w_{1} \cdot w_{3} \cdot l_{1} \cdot l_{1}: \sigma \mapsto \sigma^{\prime}=-\sigma .
\end{array}
$$

Note also that (PVI) is invariant for $\theta_{1} \mapsto-\theta_{1}$. This maps $\sigma \mapsto-\sigma$. Other actions are:

$$
\begin{gathered}
w_{1}: \quad \sigma^{\prime}=-\left(\theta_{1}+\theta_{\infty}\right) ; \quad w_{2}: \quad \sigma^{\prime}=\sigma ; \quad w_{3}: \quad \sigma^{\prime}=\theta_{1}+\theta_{\infty}-2 ; \quad w_{4}: \quad \sigma^{\prime}=\theta_{1}+\theta_{\infty}-2 . \\
x^{1}: \quad \sigma^{\prime}=\theta_{0}-\theta_{\infty} ; \quad x^{2}: \quad \sigma^{\prime}=\theta_{1}-\theta_{0} ; \quad x^{3}: \quad \sigma^{\prime}=\theta_{1}-\theta_{\infty}=\sigma .
\end{gathered}
$$

\section{Appendix 4: Examples of Taylor Expansions}

We give the solutions of (PVI) of the form $y(x)=\sum_{n=0}^{\infty} b_{n} x^{n}, b_{0} \neq 0$, depending on the value of the coefficients $\theta_{0}, \theta_{1}, \theta_{x}, \theta_{\infty}$. Similar examples can be constructed for solutions $y(x)=\sum_{n=1}^{\infty} b_{n} x^{n}$, $b_{1} \neq 0$, by the symmetry $(22)$.

We observe that, in general, the free parameter appears starting from some power $x^{m}$. If we truncate the series at $x^{m-1}$, we cannot see it.

We always denote $b_{n}$ the coefficients, though they change case by case.

* Example 1: (PVI) always has one solution (18) when $\theta_{\infty}-\theta_{1} \notin \mathbf{Z}$, and one solution (21) when $\theta_{\infty}+\theta_{1} \notin \mathbf{Z}$.

* Example 2: Case $\theta_{1}+\theta_{\infty}=0$.

i) There are solutions (19), if $\theta_{x}= \pm \theta_{0}, \theta_{\infty} \neq 1$.

ii) There is a solution (18), defined for $\theta_{\infty} \neq 1, \frac{2 n+1}{2}$, where $n \in \mathbf{Z}$. The condition $\theta_{\infty} \neq \frac{2 n+1}{2}$ follows from the condition $\theta_{1}-\theta_{\infty} \neq n$ in (18), when $\theta_{1}=-\theta_{\infty}$. The denominators of the coefficients $b_{n}$ vanish for half-integer $\theta_{\infty}$, and for $\theta_{\infty}=1$.

iii) For $\theta_{\infty}=\frac{2 n+1}{2}$, solutions $y(x)=\sum_{n=0}^{\infty} b_{n} x^{n}$ in ii) are not defined. On the other hand, solutions (19) are defined, provided that $\theta_{x}= \pm \theta_{0}$. Moreover, for a given $\theta_{\infty}=\frac{2 n+1}{2}$, there may be solutions equivalent to (19), provided that $\theta_{x} \pm \theta_{0}$ assumes some integer values. For example, consider $\theta_{\infty}=\frac{3}{2}$. We have (19):

$$
y(x)=-2+a x+\left(\theta_{0}^{2}-1+\frac{3}{2} a-\frac{1}{2} a^{3}\right) x^{2}+\sum_{n=3}^{\infty} b_{n}\left(a ; \theta_{0}, \frac{3}{2}\right) x^{n}, \quad \theta_{x}= \pm \theta_{0} ;
$$

and a solution equivalent to (19):

$$
y(x)=4-\left(2 \pm \theta_{0}\right) x+a x^{2}+\sum_{n=3}^{\infty} b_{n}\left(a ; \theta_{0}\right) x^{n}, \quad \theta_{x}= \pm\left(1+\theta_{0}\right), \quad \pm\left(1-\theta_{0}\right) .
$$

iv) If $\theta_{\infty}=0,1$, we do not have any solution of the desired form, except for the singular solutions $y=0,1$. If $\theta_{\infty}=2$ we have a solution equivalent to (20). The parameter $a$ is the coefficient of $x^{3}$ (coefficients of $x^{0}, x$ and $x^{2}$ have no parameter).

* Example 3: Case $\theta_{\infty}-\theta_{1}=0$.

i) We have solutions (19)

ii) We have the solution (21), with the substitution: $\theta_{1}=\theta_{\infty}$. This is defined for $\theta_{\infty} \neq 1, \frac{2 n+1}{2}$.

iii) For $\theta_{\infty}=\frac{2 n+1}{2}$ we find solutions of exactly the form (19), when $\theta_{x}= \pm \theta_{0}$. Moreover, for any given $\theta_{\infty}=\frac{2 n+1^{2}}{2}$, there may be solutions equivalent to (19), provided that $\theta_{x} \pm \theta_{0}$ has some integer 
value. For example, consider $\theta_{\infty}=-\frac{1}{2}$. There are solutions (19):

$$
y(x)=\frac{2}{3}+a x+\sum_{n=2}^{\infty} b_{n}\left(a ; \theta_{0},-\frac{1}{2}\right) x^{n}, \quad \theta_{x}= \pm \theta_{0} ;
$$

and the equivalent solutions:

$$
y(x)=\frac{4}{3}-\frac{2 \pm \theta_{0}}{9} x+a x^{2}+\sum_{n=3}^{\infty} b_{n}\left(a ; \theta_{0}\right) x^{n}, \quad \theta_{x}= \pm\left(1+\theta_{0}\right) \text { or } \pm\left(1-\theta_{0}\right) .
$$

Another example: consider $\theta_{\infty}=\frac{1}{2}$. In this case we just have (19), or the singular solution $y=1$.

iv) For $\theta_{\infty}=0,1$ we don't have solutions of the desired form, except for the singular solutions $y=0,1$. If $\theta_{\infty}=2$ we have a solution equivalent to (20). The parameter $a$ is the coefficient of $x^{3}$ (coefficients of $x^{0}, x$ and $x^{2}$ have no parameter).

* Example 4: Case $\theta_{1}-\theta_{\infty}=-1$.

i) We have the solution (21), defined for $\theta_{\infty} \neq 1, \frac{2 n+1}{2}$, where $n \in \mathbf{Z}$.

ii) For any $\theta_{\infty}=\frac{2 n+1}{2}$, solutions (19) are defined, when $\theta_{0} \pm \theta_{x}=0$. Moreover, solutions may exist equivalent to (19) by symmetry, provided that $\theta_{0} \pm \theta_{x}$ is some integer.

iii) For $\theta_{\infty}=1$ we are exactly in the case (20).

iv) For $\theta_{\infty}=0,2$ we have solutions equivalent to (20):

$y(x)=2+\frac{\theta_{0}^{2}-\theta_{x}^{2}-3}{6} x+a x^{2}+\left\{\frac{\left[\left(\theta_{0}-\theta_{x}\right)^{2}-9\right]\left[\left(\theta_{0}+\theta_{x}\right)^{2}-9\right]\left(\theta_{0}^{2}-\theta_{x}^{2}\right)}{4320}+a \frac{\theta_{0}^{2}-\theta_{x}^{2}+3}{6}\right\} x^{3}+\ldots$

* Example 5: Case $\theta_{1}-\theta_{\infty}=1$.

i) We have solution (21), with the substitution of $\theta_{1}=\theta_{\infty}+1$, and defined for $\theta_{\infty} \neq 1, \frac{2 n+1}{2}, n \in \mathbf{Z}$.

ii) Equivalent to (19) by symmetry, we have the solution:

$$
\begin{gathered}
y(x)=\frac{2}{1-\theta_{\infty}}+\frac{\left(\theta_{\infty}+1\right)\left(2 \pm \theta_{0}\right)}{3\left(\theta_{\infty}-1\right)} x+a x^{2}+\sum_{n=3}^{\infty} b_{n}\left(a, \theta_{\infty}, \theta_{0}\right) x^{n}, \\
\theta_{\infty} \neq 0,1 ; \quad \theta_{x}= \pm\left(1-\theta_{0}\right) \text { or } \pm\left(1+\theta_{0}\right)
\end{gathered}
$$

The two signs in the coefficient of $x$ depend on the choice $\theta_{x}= \pm\left(1-\theta_{0}\right)$ or $\pm\left(1+\theta_{0}\right)$ respectively. Similar change of signs occur in all the coefficients $b_{n}$.

iii) If $\theta_{\infty}=0$, the solution in case ii) is not defined (denominators in the coefficients $b_{n}$ vanish). We have anyway a solution equivalent to $(20)$ :

$$
y(x)=2+\frac{\theta_{0}^{2}-\theta_{x}^{2}-3}{6} x+a x^{2}+\sum_{n=3}^{\infty} b_{n}\left(a ; \theta_{0}, \theta_{\infty}\right) x^{n} .
$$

If $\theta_{\infty}=2$, we have a solution equivalent to (20). The parameter $a$ is the coefficient of $x^{4}$ (no parameter in lower powers of $x$ ).

iv) If $\theta_{\infty}=\frac{2 n+1}{2}$ the solution in i) is not defined. Solutions exist equivalent to (19), provided that $\theta_{x} \pm \theta_{0}$ is some integer.

v) For $\theta_{\infty}=-1$ we have a solution equivalent to (20):

$$
y(x)=1+a x^{2}+\frac{a\left(\theta_{x}^{2}-\theta_{0}^{2}+3\right)}{6} x^{3}+\sum_{n=4}^{\infty} b_{n}\left(a ; \theta_{0}, \theta_{x}\right) x^{n} .
$$

vi) For $\theta_{\infty}=1$, solutions of the desired form do not exist, except for $y=1$. 
We could proceed at our pleasure, choosing any value $\theta_{\infty} \pm \theta_{1}$ integer. We would always have solutions of three kinds. 1) One out of the two solutions (18) and (21). 2) Solutions equivalent to (19) - at least when $\theta_{\infty}$ is half integer - provide that $\theta_{x} \pm \theta_{0}$ is some integer. 3) Solutions equivalent to $(20)$, for $\theta_{\infty}$ equal to some integer.

\section{References}

[1] A.V.Kitaev: An Isomonodromy Cluster of Two Regular Singularities. Sfb 288 Preprint No. 149. Teschnishe Universität, Berlin, (1994). Available in revised form at arXiv:math.CA/0606562.

[2] A.V. Andreev, A.V. Kitaev: Connection formulae for asymptotics of the fifth Painleve transcendent on the real axis. Nonlinearity, 13, (2000), 1801-1840.

[3] W.Balser, W.B.Jurkat, D.A.Lutz: Birkhoff Invariants and Stokes' Multipliers for Meromorphic Linear Differential Equations, Journal Math. Analysis and Applications, 71, (1979), 48-94.

[4] W.Balser, W.B.Jurkat, D.A.Lutz: On the reduction of connection problems for differential equations with an irregular singular point to ones with only regular singularities, SIAM J. Math. Anal., 12, (1981), 691-721.

[5] P.Boalch: From Klein to Painlevé, via Fourier, Laplace and Jimbo, math.AG/0308221 (2004)

[6] A.D.Bruno, I.V. Goryuchkina: Expansions of Solutions of the Sixth Painlevé Equation. Doklady Mathematics, 69, (2004), 733-737

[7] B.Dubrovin: Painlevé trascendents in two-dimensional topological field theory. The Painlevé Property, One Century later, R.Conte, Springer 1999.

[8] B.Dubrovin-M.Mazzocco: Monodromy of Certain Painlevé-VI trascendents and Reflection Groups. Invent. math., 141, (2000), 55-147.

[9] D.Guzzetti: On the Critical Behavior, the Connection Problem and the Elliptic Representation of a Painlevé 6 Equation. Mathematical Physics, Analysis and Geometry, 4, (2001), 293-377.

[10] D.Guzzetti: Inverse Problem and Monodromy Data for 3-dimensional Frobenius Manifolds. Mathematical Physics, Analysis and Geometry, 4, (2001), 254-291.

[11] D.Guzzetti: The Elliptic Representation of the General Painlevé 6 Equation. Communications in Pure and Applied Mathematics, 55, (2002), 1280-1363.

[12] D.Guzzetti: The Elliptic Representation of the Sixth Painleve Equation. Publications of the SMF, "Seminaires et Congres", Vol 14, (2006).

[13] N.Hitchin: Hypercomplex Manifolds and the Space of Framings, The Geometric Universe, Oxford Univ. Press, (1998), 9-30.

[14] E.L.Ince: Ordinary Differential Equations. Dover Publications.

[15] A.R.Its, V.Y.Novokshenov: The isomonodromy deformation method in the theory of Painlevé equations, Springer Lecture Notes in Mathematics 1191, (1986).

[16] K.Iwasaki, H.Kimura, S.Shimomura, M.Yoshida: From Gauss to Painleve'. Aspects of Mathematics 16, (1991).

[17] M.Jimbo: Monodromy Problem and the Boundary Condition for Some Painlevé Trascendents. Publ. RIMS, Kyoto Univ.,18 (1982), 1137-1161.

[18] M.Jimbo, T.Miwa, K.Ueno: Monodromy Preserving Deformations of Linear Ordinary Differential Equations with Rational Coefficients (I). Physica D 2, (1981), 306

[19] K.Kaneko: Painlevé VI transcendents which are meromorphic at a fixed singularity. Proceedings of the Japan Academy, Vol 82, Ser. A, No. 5 (2006), 71-76 . 
[20] K.Kaneko, Y.Ohyama: Fifth Painlevé transcendents which are analytic at the origin. To appear.

[21] M.Mazzocco: Picard and Chazy Solutions to the Painlevé VI Equation. Math. Ann., 321, no.1, (2001), 157-195.

[22] M.Mazzocco: Rational Solutions of the Painlevé VI Equation, J. Phys. A: Math. Gen. 34, (2001), 2281-2294.

[23] N.E.Norlund:

itmic Solutions of the Hypergeometric Equation. Mat.Fys.Skr.Dan.Vid.Selsk., 2, (1963), no. $5,1-58$

[24] K.Okamoto: Studies on the Painlevé Equation. I - Sixth Painlevé Equation $P_{V I}$, Ann. Mat. Pura Appl. 148, (1987), 337-381.

[25] H. Qin, Y.Lu: On the asymptotics of the real solutions of the general sixth Painleve equation . (2006)

[26] S.Shimomura: Painlevé Trascendents in the Neighbourhood of Fixed Singular Points. Funkcial.Ekvac., 25, (1982), 163-184.

Series Expansions of Painlevé Trascendents in the Neighbourhood of a Fixed Singular Point. Funkcial.Ekvac.,25, (1982), 185-197.

Supplement to "Series Expansions of Painlevé Trascendents in the Neighborhood of a Fixed Singular Point". Funkcial.Ekvac.,25, (1982), 363-371.

A Family of Solutions of a Nonlinear Ordinary Differntial Equation and its Application to Painlevé Equations (III), (V), (VI). J.Math. Soc. Japan, 39, (1987), 649-662. 